\title{
CALCULATING A GARBAGE COLLECTOR
}

\author{
Ulrich Berger \\ Mathematisches Institut, Ludwig-Maximilians-Universität \\ Theresienstr. 39, D-8000 München 2, Fed. Rep. Germany \\ Werner Meixner, Bernhard Möller \\ Institut für Informatik, Technische Universität München \\ Postfach 2024 20, D-8000 München 2, Fed. Rep. Germany
}

\section{Introduction}

In this paper we give a calculational account of a garbage collection algorithm. There have been a number of papers with a similar programme (cf. [Broy, Pepper 82], [Dewar et al. 82], [van Diepen, de Roever 86], [Möller 87]). However, these treatments either were not completely formal or suffered from using an inconvenient representation of the problem. With this paper we want to introduce partial maps and their algebraic properties as a vehicle for treating pointer structures, as dealt with in garbage collection, both conveniently and formally. Moreover, we want to develop the algorithms to a level which can actually be transcribed directly into machine code allowing the use of overwriting and the like; this was not achieved in most of the papers cited. It also turns out that the theory of finite maps allows very concise high-level specifications of the subproblems involved in garbage collection.

The situation in which garbage collection becomes necessary is the following: The store, which accomodates a large number of records referencing one another through pointers, is exhausted, i.e., there is (almost) no more free storage left for the allocation of new records. Usually there is a distinguished set of entry pointers to the pointer structure which is given by the values of the currently active variables of the program that operates on the store. Only those records reachable through chains of references from the entry pointers actually need to be saved; all other records are inaccessible and thus the corresponding storage can be reclaimed.

What does garbage collection mean in a more abstract sense? To explain this, we liberate ourselves from the concrete contents of the records in the store and consider only their interrelationship through the pointers. This leads to a graph-like structure in which the nodes correspond to the records, and the arcs correspond to the pointers. More precisely, we consider a graph to be a partial map $G$ : node $\longrightarrow$ node* that assigns to each node from a subset of the set node of all possible nodes the sequence of its successor nodes. We use sequences rather than sets, since the fields of a record are ordered and may contain repetitions. If, by some process, we can distinguish a proper subgraph $G^{\prime}$ of $G$ such that $G^{\prime}$ contains all the nodes accessible from the entry nodes, then $G$ can be said to contain garbage about which we could as well forget. In this case, garbage collection means to compute $G^{\prime}$ from $G$ and to operate on $G^{\prime}$ successively.

Getting more concrete again, we work with representations of such graphs. The task then consists in computing a representation of $G^{\prime}$ from one of $G$. In this paper we treat representations, called states, of graphs in a linear storage. For a state, the restriction to a substate usually leads to gaps in the storage; i.e., there are cells the contents of which have no meaning for the represented graph. Now, one possibility of garbage collection consists in detecting these gaps and compactifying the meaningful part by copying it to an initial interval of the storage; then a contiguous rest of the storage becomes free for further use. 
We develop a corresponding algorithm from a specification at the level of graphs together with a notion of their representation in a linear storage. Although the final algorithm is fairly intricate in that it employs various ways of chaining and unchaining certain sets of storage cells, its structure becomes clear through a modular development by transformations.

\section{Preliminaries}

\subsection{Transforming Nondeterministic Specifications}

The development in this paper is based on the applicative part of the wide spectrum language CIP-L (cf. [Bauer et al. 85]), which includes a full typed lambda-calculus as well as pre-algorithmic specification constructs such as quantification, set comprehension, and non-deterministic choice. Typed $\lambda$-abstractions are written in the form

$$
(\mathrm{m} x) \mathrm{n}: E
$$

where $\mathrm{m}$ is the argument type and $\mathrm{n}$ is the result type. Recursion is introduced by the fixpoint construction

$$
(\text { fix } x: E)
$$

where the type of $x$ is that of $E$. Declarations can then be introduced by the definitional transformation rule

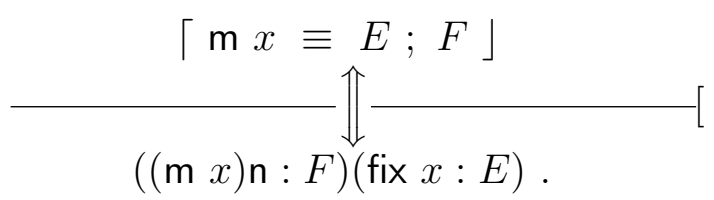

CIP-L has a mathematical semantics that associates with each expression $E$ the set $\mathcal{B} \llbracket E \rrbracket$ of possible values; $\mathcal{B} \llbracket E \rrbracket$ is called the breadth of $E$. The special value $\perp$ models the possibility of an erroneous or nonterminating computation. We set

$$
\begin{aligned}
& \mathrm{DEF} \llbracket E \rrbracket \stackrel{\text { def }}{\Leftrightarrow} \perp \notin \mathcal{B} \llbracket E \rrbracket, \\
& \mathrm{DET} \llbracket E \rrbracket \stackrel{\text { def }}{\Leftrightarrow}|\mathcal{B} \llbracket E \rrbracket|=1,
\end{aligned}
$$

and call $E$ defined resp. determinate if DEF $\llbracket E \rrbracket$ resp. DET $\llbracket E \rrbracket$ holds. For convenience, the semantical values are also considered as expressions. The details of the semantic description can be found in [Bauer et al. 85].

Based on the breadth, two fundamental relations between expressions are defined: $E_{1}$ and $E_{2}$ are called equivalent if $\mathcal{B} \llbracket E_{1} \rrbracket=\mathcal{B} \llbracket E_{2} \rrbracket$; we denote this by $E_{1} \equiv E_{2}$. This "strong" or meta-equality is not to be confused with "weak" (i.e. strict) equality tests = in the language itself: The formula

$$
\perp \equiv \perp
$$

is valid. However,

$$
\perp=\perp \not \equiv \text { true ; }
$$

rather we have

$$
\perp=\perp \equiv \perp .
$$

Equivalences are also denoted in the form of transformation rules, viz. as 


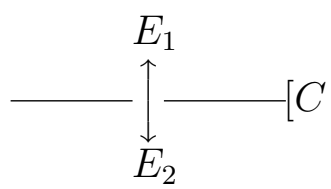

where $C$ is a (possibly empty) list of applicability conditions, i.e., of conditions sufficient for the validity of the equivalence. Similarly, $E_{2}$ is called a descendant of $E_{1}$ if $\mathcal{B} \llbracket E_{2} \rrbracket \subseteq \mathcal{B} \llbracket E_{1} \rrbracket$; we denote this by $E_{1} \supseteq E_{2}$ and, in the form of a transformation rule, as

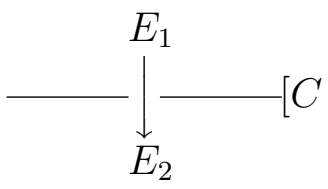

where $C$ again is a list of applicability conditions.

As an important aid in specifying and developing recursive routines we use assertions about the objects involved. They are formulated as Boolean expressions of the language. Given such an expression $P$, we use the notation

$$
P \triangleright E
$$

as an abbreviation for the expression

$$
\text { if } P \text { then } E \text { else } \perp \text { fi . }
$$

A collection of useful algebraic properties of this construct can be found in [Möller 89]. Our principal use of it is within parameter restrictions for functions (cf. [Bauer, Wössner 82, Bauer et al. 85]): Let $R$ be a Boolean expression possibly involving the identifier $x$. Then the declaration

$$
\text { funct } f \equiv(\mathrm{m} x: R) \mathrm{n}: E
$$

of function $f$ with parameter $x$ restricted by $R$ and with body $E$ is by definition equivalent to

$$
\text { funct } f \equiv(\mathrm{m} x) \mathrm{n}: R \triangleright E \text {. }
$$

This means that $f$ is undefined for all arguments $x$ that violate the restriction $R$. If $f$ is recursive, $R$ has to hold also for the parameters of the recursive calls to ensure definedness; hence in this case $R$ corresponds to invariants as known from imperative programming.

We now want to develop our central transformation rule for obtaining descendants of nondeterministic functions. Consider a function abstraction

$$
(\mathrm{m} x) \mathrm{n}: E<x, f>
$$

and the corresponding recursion

$$
R \widehat{=}(\text { fix } f:(\mathrm{m} x) \mathrm{n}: E<x, f>) .
$$

The notation $E<x, f>$ indicates that only the free identifiers $x$ and $f$ are of interest in $E$; for expressions $A$ and $G$ then $E<A, G>$ is a shorthand for the substitution $E \llbracket A, G$ for $x, f \rrbracket$. We want to find a criterion under which the recursion $R$ is a descendant (and hence a correct implementation) of some function $F$ which may be viewed as the specification. The idea is to employ noetherian induction on the arguments of $F$ and $R$. Let therefore $\prec$ be a determinate expression of kind funct $(\mathrm{m}, \mathrm{m})$ bool that denotes a noetherian strict-order on the set of values of kind $\mathrm{m}$ different from $\perp$. We define, for arbitrary function $g$ of kind funct $(\mathrm{m}) \mathrm{n}$,

$$
\text { BELOW } \llbracket x, g \rrbracket<y>\stackrel{\text { def }}{\equiv} y \prec x \triangleright g(y) \text {. }
$$


This denotes an expression that agrees with $g(y)$ for $y \prec x$ and is undefined for all other $y$; hence it corresponds to a restriction of $g$ to values strictly less than $x$. Now we call $E<x, f>$ recurrent wrt. $\prec$ iff

$$
E<x, g>\equiv E \ll x, \mathrm{BELOW} \llbracket x, g \rrbracket \gg,
$$

where $g$ is a fresh identifier and $E \ll x$, BELOW $\llbracket x, g \rrbracket \gg$ results from $E<x, g>$ by replacing all calls $g(A)$ by BELOW $\llbracket x, g \rrbracket<A>$. This means that for all $g$ the value of $E<x, g\rangle$ depends at most on values $g(y)$ with $y \prec x$. We call $E<x, f>$ recurrent if there is some $\prec$ such that $E<x, f>$ is recurrent wrt. $\prec$. Then we have

\section{Theorem 2.1.1}

The following rule (DESCENDANT-FIXPOINT) is correct:

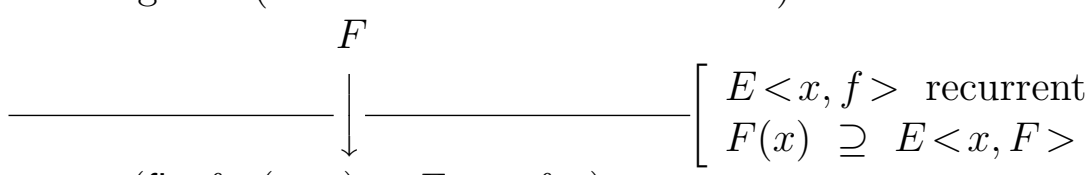

$($ fix $f:(\mathrm{m} x) \mathrm{n}: E<x, f>)$

Proof: We abbreviate the output scheme again to $R$. Let $E<x, f\rangle$ be recurrent w.r.t $\prec$ and assume $F(x) \supseteq E<x, F>$. We show $F(u) \supseteq R(u)$ by noetherian induction on the values $u \not \equiv \perp$ of kind $\mathrm{m}$. By induction hypothesis we may assume $F(v) \supseteq R(v)$ for all $v \prec u$, and thus

$$
\mathrm{BELOW} \llbracket u, F \rrbracket \supseteq \mathrm{BELOW} \llbracket u, R \rrbracket .
$$

Then

$$
\begin{aligned}
& F(u) \\
& \supseteq E<u, F> \\
& \equiv E \ll u, \mathrm{BELOW} \llbracket u, F \rrbracket \gg \text { (since } E<x, f>\text { is recurrent) } \\
& \supseteq E \ll u, \mathrm{BELOW} \llbracket u, R \rrbracket \gg \text { (by }(+) \text { and } \supseteq \text {-monotonicity of } E<x, f>\text { ) } \\
& \equiv E<u, R> \\
& \equiv R(u) \\
& \text { (since } E<x, f>\text { is recurrent) } \\
& \text { (by definition of } R \text { ). }
\end{aligned}
$$

Finally, since all expressions of kind funct $(m) n$ denote strict functions in CIP-L, we have $F(\perp) \equiv \perp \equiv R(\perp)$ and thus also $F(\perp) \supseteq R(\perp)$.

Note that the descendant relation implies that for totally defined $F$ the recursively defined function $f$ always terminates.

\subsection{Notations for Specific Data Structures}

\subsubsection{Sequences}

Given a set $M$, we denote by $M^{*}$ the set of all finite sequences of $M$-elements. The empty sequence is denoted by $\diamond$. Then $M^{+} \stackrel{\text { def }}{=} M^{*} \backslash\{\diamond\}$ is the set of all nonempty finite sequences of $M$ elements. The singleton sequence consisting just of $x \in M$ is denoted by $\langle x\rangle$. Concatenation of sequences is denoted by + . Accordingly, for sequences $\alpha_{i}$ we write

$$
\sum_{i \in[1: n]} \alpha_{i}
$$

for

$$
\alpha_{1}+\ldots+\alpha_{n} \text {. }
$$


We write $x \in \alpha$ to express that $x$ occurs in the sequence $\alpha$. Moreover, $\operatorname{set}(\alpha) \stackrel{\text { def }}{\equiv}\{x \mid x \in \alpha\}$. The length of a sequence $\alpha$ is denoted by $|\alpha|$. For $0<i \leq|\alpha|$ we write $\alpha[i]$ for the $i$-th element of $\alpha$.

Given a function $f: M \longrightarrow N$ we denote by $f *$ its unique homomorphic extension mapping $M^{*}$ to $N^{*}$, i.e.,

$$
f *\left(<x_{1}, \ldots, x_{n}>\right) \equiv<f\left(x_{1}\right), \ldots, f\left(x_{n}\right)>
$$

\subsubsection{Sets and Orders}

The cardinality of a set $M$ is denoted by $|M|$. Moreover, the complement of a subset $N$ of $M$ is denoted by $\bar{N}$.

For a finite subset $s \subseteq M$ of a linearly ordered set $M$ we denote by $\operatorname{sort}(s)$ the unique repetitionfree sequence that contains exactly the elements of $s$ in ascending order from left to right. $M$ is well-ordered if every nonempty subset $s \subseteq M$ contains a least element $\min (s)$. If then $M$ also contains a greatest element $\infty$, we set

$$
\inf (s) \stackrel{\text { def }}{\equiv} \min (s \cup\{\infty\}) .
$$

The greatest element of $s$ (if any) is denoted by $\max (s)$. If $M$ contains a least element $-\infty$ we set

$$
\sup (s) \stackrel{\text { def }}{\equiv} \max (s \cup\{-\infty\}) .
$$

Define for a subset $s \subseteq M$ of an ordered set $M$

$$
\begin{array}{cl}
s^{\vee} & \stackrel{\text { def }}{\equiv}\{y \mid \exists x \in s: x \leq y\} \\
s^{\wedge} & \stackrel{\text { def }}{\equiv}\{y \mid \exists x \in s: y \leq x\} \\
\text { isinterval }(s) & \stackrel{\text { def }}{\Leftrightarrow} s \equiv s^{\vee} \cap s^{\wedge} .
\end{array}
$$

$s$ is an initial interval of $M$ if $s^{\wedge} \equiv s$. For abbreviation we write $\stackrel{\vee}{x}, \hat{x}$ instead of $\{x\}^{\vee},\{x\}^{\wedge}$. Moreover, we set

$$
\begin{aligned}
& {[x: y] \stackrel{\text { def }}{\equiv} \stackrel{\vee}{x} \cap \hat{y},} \\
& {[x: y[\stackrel{\text { def }}{=}[x: y] \backslash\{y\},} \\
& ] x: y] \stackrel{\text { def }}{\equiv}[x: y] \backslash\{x\}, \\
& ] x: y[\stackrel{\text { def }}{=}[x: y] \backslash\{x, y\} .
\end{aligned}
$$

Suppose again that $M$ is well-ordered and contains a greatest element $\infty$. Then for $s \subseteq M$ and $x \in M$ we define

$$
\operatorname{succ}_{s}(x) \stackrel{\text { def }}{=} \inf (s \backslash\{x\}) \text {. }
$$

\subsection{Relations and Maps}

\subsubsection{Basic Notions}

A binary relation $r$ between sets $M$ and $N$ is a subset $r \subseteq M \times N$. We write $\downarrow r$ and $\uparrow r$ for domain and range of $r$, resp. Moreover, we define

$$
\operatorname{set}(r) \stackrel{\text { def }}{\equiv} \downarrow r \cup \uparrow r .
$$


For $s \subseteq M$ we denote by $s \uparrow r$ the image of $s$ under $r$. Likewise, $t \downarrow r$ is the inverse image of $t \subseteq N$ under $r$.

A (partial) map $m$ from a set $M$ to a set $N$ is a relation $m \subseteq M \times N$ such that $(x, y) \in$ $m \wedge(x, z) \in m \Rightarrow y \equiv z$. Some of our notation derives from this set view of maps. E.g., by $\emptyset$ we denote the empty partial map from $M$ to $N$.

Next, we define

$$
[s \mapsto y] \stackrel{\text { def }}{=}\{(x, y) \mid x \in s\} ;
$$

this is the constant map assigning $y$ to every element of $s$. In this construction, $y$ frequently is obtained by applying another map $m$. To cope in an algebraically convenient way with partialities, we set

$$
[s \mapsto m(x)] \stackrel{\text { def }}{=} \emptyset \quad \text { for } x \notin \downarrow m .
$$

Symmetrically, we also define

$$
[\{n(x)\} \mapsto y)] \stackrel{\text { def }}{=} \emptyset \quad \text { for } x \notin \downarrow n
$$

In using these notations we omit singleton set braces, i.e., we write $x \uparrow m, y \downarrow m$, and $[x \mapsto y]$ instead of $\{x\} \uparrow m,\{y\} \downarrow m,[\{x\} \mapsto y]$. Note that $[x \mapsto y] \equiv\{(x, y)\}$.

\section{Lemma 2.3.1 (Functionality)}

Let $m$ be a map.

(1) $|m| \equiv|\downarrow m|$.

(2) $t_{1} \cap t_{2} \equiv \emptyset \Rightarrow t_{1} \downarrow m \cap t_{2} \downarrow m \equiv \emptyset$.

A map is injective if the predicate isinjective $(m)$ holds, where

$$
\operatorname{isinjective}(m) \stackrel{\text { def }}{\Leftrightarrow} \forall x, y \in \downarrow m: m(x) \equiv m(y) \Rightarrow x \equiv y \text {. }
$$

In this case we denote its inverse map by $m^{-1}$.

\section{Lemma 2.3.2}

$$
\text { isinjective }(m) \Leftrightarrow \forall s, t:(s \cap t) \uparrow m \equiv s \uparrow m \cap t \uparrow m .
$$

By $\circ$ we denote the composition of partial maps:

$$
s \uparrow(n \circ m) \equiv(s \uparrow m) \uparrow n .
$$

\subsubsection{Map Union}

Two maps $m, n: M \longrightarrow N$ are compatible if $m(x) \equiv n(x)$ for all $x \in \downarrow m \cap \downarrow n$. In particular this holds if $\downarrow m \cap \downarrow n \equiv \emptyset$. For compatible $m, n$ their union $m \cup n$ is again a map. This generalizes to families $\left(m_{i}\right)_{i \in I}$ of maps ( $I$ may even be infinite) if the maps $m_{i}$ are pairwise compatible; we then write $\bigcup_{i \in I} m_{i}$ for the union map. If $I \equiv \emptyset$, we set $\bigcup_{i \in I} m_{i} \equiv \emptyset$ as well. 


\section{Lemma 2.3.3}

$$
\begin{aligned}
& \text { (1) } m \equiv \bigcup_{x \in \downarrow m}[x \mapsto m(x)] \quad \text { (domain-oriented representation) } \\
& \text { (2) } m \equiv \bigcup_{z \in \uparrow m}[(z \downarrow m) \mapsto z] \quad \text { (range-oriented representation) } \\
& \text { (3) } k \circ \bigcup_{i \in I} m_{i} \equiv \bigcup_{i \in I}\left(k \circ m_{i}\right) \\
& \text { (4) } \quad\left(\bigcup_{i \in I} m_{i}\right) \circ k \equiv \bigcup_{i \in I}\left(m_{i} \circ k\right) \\
& \text { (5) } \text { isinjective }(m) \Rightarrow m^{-1} \equiv \bigcup_{x \in \downarrow m}[m(x) \mapsto x]
\end{aligned}
$$

We want to develop a recursive routine for calculating particular unions over finite, well-ordered index sets. The specification reads

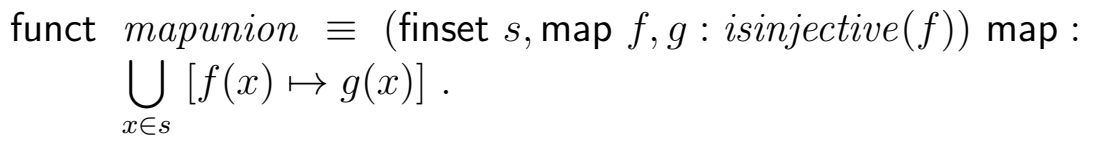

The parameter restriction serves to make the union well-defined. If $s \equiv \emptyset$, by definition mapunion $(s, f, g) \equiv \emptyset$. Otherwise, for arbitrary $z \in s$ we have $s \equiv\{z\} \cup s \backslash\{z\}$ and hence

$$
\begin{aligned}
& \bigcup_{x \in\{z\} \cup s \backslash\{z\}}^{\operatorname{mapunion}(s, f, g)}[f(x) \mapsto g(x)] \\
\equiv & \bigcup_{x \in\{z\}}[f(x) \mapsto g(x)] \cup \bigcup_{x \in s \backslash\{z\}}[f(x) \mapsto g(x)] \\
\equiv & {[f(z) \mapsto g(z)] \cup \operatorname{mapunion}(s \backslash\{z\}, f, g) . }
\end{aligned}
$$

In particular, this holds for $z \equiv \min (s)$. Thus we obtain the recursion (for well-founded $M$ termination is obvious)

$$
\begin{aligned}
& \text { funct mapunion } \equiv \text { (finset } s \text {, map } f, g \text { : isinjective }(f)) \text { map : } \\
& \text { if } s=\emptyset \text { then } \emptyset \\
& \text { else } \mathrm{m} z \equiv \min (s) ; \\
& \quad[f(z) \mapsto g(z)] \cup \operatorname{mapunion}(s \backslash\{z\}, f, g) \quad \mathrm{fi} .
\end{aligned}
$$

To make this into a tail-recursion and to avoid the repeated use of min, we assume that $\mathrm{m}$ has a greatest element $\infty$ and define the embedding

$$
\text { funct } \begin{aligned}
& m u \equiv(\text { finset } s, \operatorname{map} f, g, \operatorname{map} r, \mathrm{~m} x: \\
&\quad \text { isinjective }(f) \wedge x=\min (s)) \text { map : } \\
& r \cup \operatorname{mapunion}(s, f, g) .
\end{aligned}
$$

We have

$$
\operatorname{mapunion}(s, f, g) \equiv \operatorname{mu}(s, f, g, \emptyset, \min (s))
$$

Now we calculate

$$
\begin{aligned}
& \operatorname{mu}(s, f, g, r, x) \\
& \equiv \text { isinjective }(f) \wedge x=\min (s) \triangleright \\
& r \cup \text { if } s=\emptyset \text { then } \emptyset \\
& \text { else } \mathrm{m} z \equiv \min (s) ; \\
& {[f(z) \mapsto g(z)] \cup \operatorname{mapunion}(s \backslash\{z\}, f, g) \quad \mathrm{fi}} \\
& \equiv \text { isinjective }(f) \wedge x=\min (s) \triangleright \\
& \text { if } s=\emptyset \text { then } r \cup \emptyset \\
& \text { else } \mathrm{m} z \equiv \min (s) ;
\end{aligned}
$$




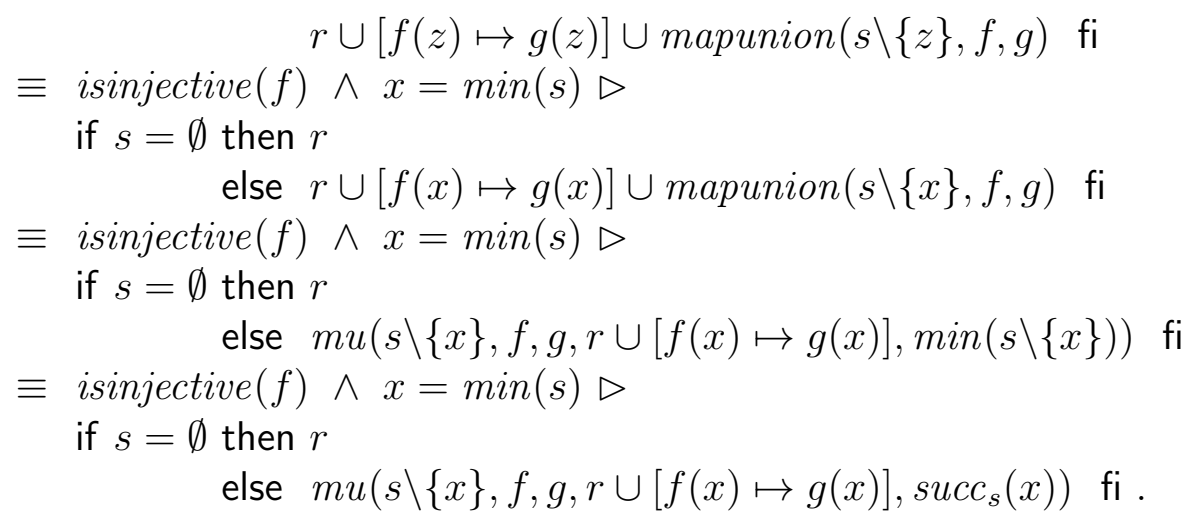

Since the termination behaviour has not changed, we are left with

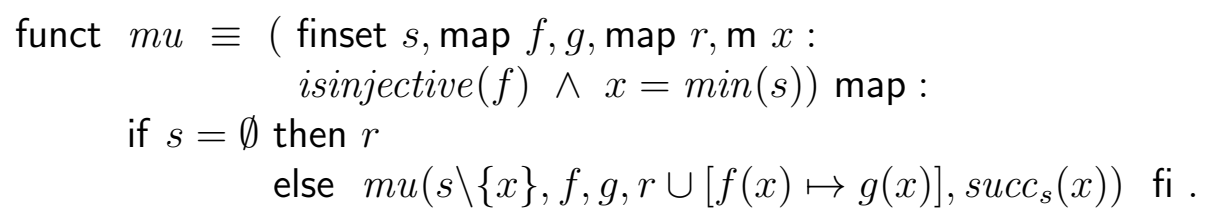

\subsubsection{Restriction and Overwriting}

The restriction of a map $m: M \longrightarrow N$ to a set $s \subseteq M$ is

$$
m \mid s \stackrel{\text { def }}{=} m \cap(s \times N) \text {. }
$$

Moreover,

$$
m \ominus s \stackrel{\text { def }}{\equiv} m \mid \bar{s} .
$$

Here again we omit singleton set braces, i.e., we write $m \ominus x$ instead of $m \ominus\{x\}$.

Another useful operation is map overwriting: Given maps $m, n: M \longrightarrow N$ we define

$$
m \triangleleft n \stackrel{\text { def }}{\equiv}(m \ominus \downarrow n) \cup n
$$

Hence,

$$
(m \varangle n)(x) \equiv \text { if } x \in \downarrow n \text { then } n(x) \text { else } m(x) \text { fi. }
$$

\section{Lemma 2.3.4}

1. $(m \ominus s) \cup n \equiv m \varangle n$ provided $\downarrow m \cap s \equiv \downarrow m \cap \downarrow n$.

2. $l \nLeftarrow(m \cup n) \equiv l \nLeftarrow n$ provided $m \subseteq l$ and $m$ and $n$ are compatible (Annihilation).

A number of further properties of these operations can be found in the appendix; we shall use them freely without explicit reference.

\section{Storage Graphs and Their Representation}

\subsection{Storage Graphs}

Storage graphs are intended to model the accessibility relations between records as given by the pointers in the records. Since the fields of a record are ordered and may contain repetitions of pointers, the usual notion of a directed graph where each node is connected to a set of successor 
nodes is not adequate for our purposes. Rather we consider sequences of successor nodes. Also, since we shall have to deal with arbitrary parts of such storage graphs, we generalize in another direction by allowing the successor map to "leave" the part under consideration.

Let node be a set of "nodes". A pseudo-graph is a partial map $G$ : node $\longrightarrow$ node*. The nodes in $G(x)$ are called the immediate successors of the node $x \in \downarrow G$. We set

$$
\operatorname{nodes}(G) \stackrel{\text { def }}{=} \downarrow G \cup \bigcup_{x \in \downarrow G} \operatorname{set}(G(x)) .
$$

A storage graph then is a pseudo-graph $G$ such that nodes $(G) \subseteq \downarrow G$. The more general case of pseudo-graphs models "dangling references" which will occur e.g. during the copying phase of our garbage collection algorithm when only part of the accessible cells have been copied to their new locations.

\subsection{Memories and Allocations}

We also want to talk about the representation of such pseudo-graphs in a (linear) memory. A memory is a pair $\mathcal{M} \equiv$ (cell, $\square$ ), where cell is a denumerable set of cells and $\square \in$ cell is a distinguished cell called the anchor of the memory.

A (partial) map $m:$ cell $\longrightarrow$ cell is called a state of $\mathrm{M}$ if the predicate isstate $(m)$ holds where

$$
\operatorname{isstate}(m) \stackrel{\text { def }}{=} \square \notin \downarrow m \text {. }
$$

A linear memory is a memory $\mathcal{M} \equiv($ cell, $\square)$ such that cell is linearly ordered by some ordering $\leq$ in which $\square$ is the least element. In the sequel we assume cell to be the set $\mathbb{N}$ of natural numbers and $\square \equiv 0$.

Let now $G$ be a pseudo-graph. We want to represent $G$ by a state of a linear memory. The idea is to store each node of $G$ together with its successors in a block of contiguous cells; the node itself is marked by cell contents $\square$. In practice, this leading cell frequently is used for storing information about a record, such as its length, type information, and the like. However, as stated in the introduction, we abstract from such details; $\square$ seems an adequate substitute here.

Let $x \in \downarrow G$. Then the size of the corresponding block is given by

$$
\operatorname{size}(x) \stackrel{\text { def }}{=} 1+|G(x)| \text {. }
$$

An allocation of $G$ is a partial map $g$ : node $\longrightarrow$ cell such that $\operatorname{nodes}(G) \subseteq \downarrow g$ and $\square \notin \uparrow g . g$ is supposed to assign to each node in $\downarrow G$ the starting cell of its block; the additional condition ensures that $\square$ can in fact be used to characterize block beginnings. Given an allocation $g$ of $G$ we define for $x \in \downarrow G$

$$
\operatorname{block}(x, g) \stackrel{\text { def }}{\equiv}[g(x) \mapsto \square] \cup \bigcup_{i \in[1:|G(x)|]}[(g(x)+i) \mapsto g(G(x)[i])] .
$$

We set

$$
\text { fields }(x, g) \stackrel{\text { def }}{\equiv}(\downarrow \operatorname{block}(x, g)) \backslash\{g(x)\} \equiv\{g(x)+i \mid i \in[1:|G(x)|]\} \text {. }
$$

We have

Lemma 3.2.1

$$
\text { isinterval }(\downarrow \operatorname{block}(x, g)) \text {. }
$$


An allocation $g$ of $G$ is overlap-free if

$$
x \not \equiv y \Rightarrow \downarrow \operatorname{block}(x, g) \cap \downarrow \operatorname{block}(y, g) \equiv \emptyset \text {. }
$$

For an overlap-free allocation we can extend the function block to sets $s \subseteq \downarrow G$ by setting

$$
\operatorname{block}(s, g) \stackrel{\text { def }}{=} \bigcup_{x \in s} \operatorname{block}(x, g) \text {. }
$$

Then the following state is a representation of $G$ :

$$
\operatorname{blockrep}(G, g) \stackrel{\text { def }}{\equiv} \operatorname{block}(\downarrow G, g) .
$$

\section{Lemma 3.2.2}

Let $G_{1}, G_{2} \subseteq G$ and let $g$ be an allocation of $G$. Then

$$
\text { blockrep }\left(G_{1} \cup G_{2}, g\right) \equiv \operatorname{blockrep}\left(G_{1}, g\right) \cup \operatorname{blockrep}\left(G_{2}, g\right) \text {. }
$$

We call a state $m$ a pseudo-graph state if the predicate ispgstate $(m)$ holds where

$$
\text { ispgstate }(m) \stackrel{\text { def }}{\Leftrightarrow} \exists G, g: m \equiv \operatorname{blockrep}(G, g) \text {. }
$$

Then

$$
\operatorname{keys}(m) \stackrel{\text { def }}{=} \square \downarrow m \equiv(\downarrow G) \uparrow g
$$

denotes the set of keys of $m$, i.e., the set of addresses that are the beginnings of blocks. We set

$$
\operatorname{arcs}(m) \stackrel{\text { def }}{\equiv} m \ominus \operatorname{keys}(m) \text {. }
$$

Moreover we define

$$
\text { followers }(m, y) \equiv \text { if } y+1 \in \downarrow \text { arcs }(m) \text { then }<y+1>+ \text { followers }(m, y+1) \text { else } \diamond \text { fi. }
$$

\section{Lemma 3.2.3}

Let $g$ be an overlap-free allocation of $G$ and let $m \equiv \operatorname{blockrep}(G, g)$.

1. $\square \notin \downarrow m \wedge \uparrow m \cap \downarrow m \subseteq \operatorname{keys}(m)$.

2. $g$ is injective.

3. $\operatorname{arcs}(m) \equiv \bigcup_{x \in \text { keys }(m)}$ followers $(m, x)$.

4. For all $x \in \downarrow G$ we have fields $(x, g) \equiv \operatorname{set}($ followers $(m, g(x)))$.

5. $\downarrow G \equiv \operatorname{keys}(m) \downarrow g \quad$ and

$$
G \equiv \bigcup_{x \in \text { keys }(m)}\left[x \downarrow g \mapsto g^{-1} *(m * \text { followers }(m, x))\right] \text {. }
$$

1. means that $\square$ in $m$ designates an "improper" cell that does not contain a value. 4 . and 5 . show how a graph can be reconstructed from its block representation.

Assume now that the set $\downarrow G$ of nodes is linearly ordered by some order $\leq$. Then an allocation $g$ is called order-preserving if

$$
\forall x, y \in \downarrow G: x \leq y \Rightarrow g(x) \leq g(y) .
$$




\section{Lemma 3.2.4}

Let $g$ be an injective and order-preserving allocation. Then for $x, y \in \downarrow G$ we have

1. $x<y \Rightarrow g(x)<g(y)$.

2. $x \leq y$ iff $g(x) \leq g(y)$, i.e., $\downarrow G$ and $\uparrow g$ are order-isomorphic.

Proof: 1 . is immediate from the injectivity of $g$.

2. We only need to show $(\Leftarrow)$. Assume $g(x) \leq g(y)$ but $x \not \leq y$. By linearity of $\leq$ then $y<x$ and hence also $g(y)<g(x)$ by 1 . Contradiction!

This lemma holds for arbitrary order-preserving injections between linear orders. For the special case of storage linearization we get

\section{Lemma 3.2.5}

Let $g$ be an overlap-free and order-preserving allocation. Then

$$
x<y \Rightarrow \downarrow \operatorname{block}(x, g)<\downarrow \operatorname{block}(y, g),
$$

where for subsets $s, t$ of an ordered set

$$
s<t \stackrel{\text { def }}{\Leftrightarrow} \forall x \in s: \forall y \in t: x<y \text {. }
$$

Proof: Since $g$ is order-preserving, $g(x)<g(y)$. Let now $u \in \downarrow$ block $(x, g)$ and $v \in \downarrow$ block $(y, g)$ and assume $v \leq u$. Since $g(y) \leq v$, we have then $g(x) \leq g(y) \leq u$ and hence $g(y) \in \downarrow$ block $(x, g)$ by isinterval $(\downarrow$ block $(x, g))$. But then $\downarrow$ block $(x, g) \cap \downarrow$ block $(y, g) \not \equiv \emptyset$, a contradiction.

We now want to characterize contiguous block representations. Call an overlap-free allocation $g$ of $G$ gap-free if isinterval $(\downarrow$ blockrep $(G, g))$ holds. $g$ is called perfect if it is overlap-free, orderpreserving, and gap-free.

\section{Theorem 3.2.6}

Let $g:$ node $\longrightarrow$ cell be a perfect allocation of $G$ and $x \in \downarrow G$ such that $x$ is not the maximum of $\downarrow G$. Then

$$
g\left(\operatorname{succ}_{\downarrow}(x)\right) \equiv g(x)+\operatorname{size}(x) .
$$

Proof: Since $g$ is order-preserving and injective, we have $g(x)<g\left(\operatorname{succ}_{\downarrow}(x)\right)$. By the above lemma then $\downarrow$ block $(x, g)<\downarrow$ block $\left(\operatorname{succ}_{\downarrow_{G}}(x), g\right)$ and in particular $\downarrow$ block $(x, g)<\left\{\right.$ succ $\left._{\downarrow}(x)\right\}$. Let

$$
z \stackrel{\text { def }}{\equiv} \max (\downarrow \text { block }(x, g)) \equiv g(x)+|G(x)| \text {. }
$$

Assume $z<u<g\left(\operatorname{succ}_{\downarrow G}(x)\right)$ for some $u$. By isinterval $(\downarrow$ blockrep $(G, g))$ we get $u \in$ $\downarrow$ blockrep $(G, g)$, say $u \in \downarrow$ block $(w, g)$ for some $w \in \downarrow G$. Then $g(w) \leq u<g\left(\operatorname{succ}_{\downarrow G}(x)\right)$ and hence $w<\operatorname{succ}_{\downarrow G}(x)$ since $g$ is order-preserving and injective. This is equivalent to $w \leq x$. But then $\downarrow$ block $(w, g) \leq \downarrow$ block $(x, g)$ contradicting $g(x) \leq z<u \in \downarrow \operatorname{block}(w, g)$. Therefore

$$
g\left(\operatorname{succ}_{\downarrow}(x)\right) \equiv z+1 \equiv g(x)+|G(x)|+1 \equiv g(x)+\operatorname{size}(x) .
$$




\section{Corollary 3.2.7}

Let $g:$ node $\longrightarrow$ cell be a perfect allocation of $G$ and $x \in \downarrow G$ such that $|\stackrel{\vee}{x} \cap \downarrow G|>i$.

Then

$$
g\left(\operatorname{succ}_{\downarrow}^{i}(x)\right) \equiv g(x)+\sum_{j<i} \operatorname{size}\left(\operatorname{succ}_{\downarrow_{G}}^{j}(x)\right) .
$$

Proof: Induction on $i$.

A pseudo-graph state $m$ is called compressed if the predicate iscgstate $(m)$ holds, where

$$
\text { iscgstate }(m) \stackrel{\text { def }}{\Leftrightarrow} \text { ispgstate }(m) \wedge \downarrow m \equiv(\downarrow m)^{\wedge} \backslash\{\square\},
$$

i.e., if its domain is an initial interval of cell $\backslash\{\square\}$. By the above corollary, given a pseudo-graph $G$ there is exactly one perfect allocation $g$ of $G$ such that $\operatorname{blockrep}(G, g)$ is compressed; $g$ is called the compressing allocation of $G$.

\section{The Garbage Collection Problem}

\subsection{The Reachable Subgraph}

When garbage collection becomes necessary, there is a set of immediate entries into the store. All blocks reachable from these entries need to be saved whereas everything else is garbage to be removed. We first treat the reachability problem at the level of storage graphs.

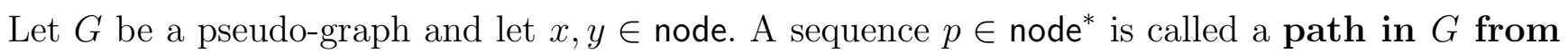
$x$ to $y$ iff the predicate $\operatorname{ispath}_{G}(p, x, y)$ holds, where

$$
\begin{aligned}
\operatorname{ispath}_{G}(p, x, y) \stackrel{\text { def }}{\Leftrightarrow} & p \in \operatorname{node}^{+} \wedge \operatorname{set}(p) \backslash\{\operatorname{last}(p)\} \subseteq \downarrow G \wedge \\
& x=\operatorname{first}(p) \wedge y=\operatorname{last}(p) \wedge \\
& \forall i \in[1:|p|-1]: p[i+1] \in G(p[i]) .
\end{aligned}
$$

We define

$$
\text { nodeset } \stackrel{\text { def }}{=}\{s \mid s \subseteq \text { node } \wedge|s|<\infty\} \text {. }
$$

Given a pseudo-graph $G$, a node $x \in \operatorname{nodes}(G)$ is reachable from some set $s \in$ nodeset iff the predicate isreachable $_{G}(x, s)$ holds where

$$
\text { isreachable }_{G}(x, s) \stackrel{\text { def }}{\Leftrightarrow} \exists z \in s, p \in \operatorname{node}^{+}: \operatorname{ispath}_{G}(p, z, x)
$$

The function rnset $_{G}:$ nodeset $\longrightarrow$ nodeset, defined by

$$
\text { rnset }_{G}(s) \stackrel{\text { def }}{\equiv}\left\{x \in \text { node } \mid \text { isreachable }_{G}(x, s)\right\}
$$

computes the set of nodes reachable from a given set.

Now, for a storage graph $G$ and a set $s \subseteq \downarrow G$, the subgraph of $G$ reachable from $s$ is $G_{s} \stackrel{\text { def }}{\equiv} G \mid \operatorname{rnset}_{G}(s)$. It is easily verified that the pseudo-graph $G_{s}$ indeed is a storage graph.

\subsection{Statement of the Garbage Collection Problem}

Consider a storage graph $G$ with a perfect allocation $g$ and a set $s \subseteq \downarrow G$. Moreover, set $n \stackrel{\text { def }}{=}$ blockrep $(G, g)$. The problem consists in computing the reachable subgraph $G_{s}$ together with the compressing allocation $g_{s}$ of $G_{s}$ as well as the corresponding state $n_{s} \stackrel{\text { def }}{\equiv}$ blockrep $\left(G_{s}, g_{s}\right)$. In fact, ultimately we are interested in an algorithm that computes $n_{s}$ directly from $n$. 


\subsection{A First Analysis of the Problem}

Assume $G, g, n$ and $G_{s}, g_{s}, n_{s}$ as in Section 4.2. Define

$$
n_{1} \stackrel{\text { def }}{=} \operatorname{blockrep}\left(G_{s}, g\right) \text {. }
$$

Thus, $n_{1}$ is the accessible but not yet compressed part of the storage. To compute $n_{s}$ from $n_{1}$, define a collapsing map $k: \downarrow n_{1} \longrightarrow$ cell by

$$
k(g(x)+i) \stackrel{\text { def }}{=} g_{s}(x)+i
$$

for $x \in \downarrow G_{s}$ and $0 \leq i \leq\left|G_{s}(x)\right|$.

\section{Lemma 4.3.1}

(1) $k$ is well-defined. Moreover, $k$ is an order-embedding and $\uparrow k \equiv \downarrow n_{s}$ (which is an initial interval of cell).

(2) $n_{s} \circ k \equiv k \circ n_{1}$.

Proof: (1) is obvious.

$$
\begin{aligned}
& n_{s}(k(g(x)+i)) \\
\equiv & n_{s}\left(g_{s}(x)+i\right) \\
\equiv & g_{s}(G(x)[i]) \\
\equiv & k(g(G(x)[i])) \\
\equiv & k\left(n_{1}(g(x)+i)\right)
\end{aligned}
$$

The preceding considerations suggest a decomposition of the problem into the following parts:

1. Compute $G_{s}$ from $G$ and $s$ (reachability).

2. Compute $n_{c} \equiv \operatorname{blockrep}\left(G_{s}, g\right)$ from $G_{s}$ and $g$.

3. Compute $k$ from $n_{c}$.

4. Compute $n_{s}$ from $n_{c}$ and $k$ (copying).

Diagrammatically the situation can be described as follows:

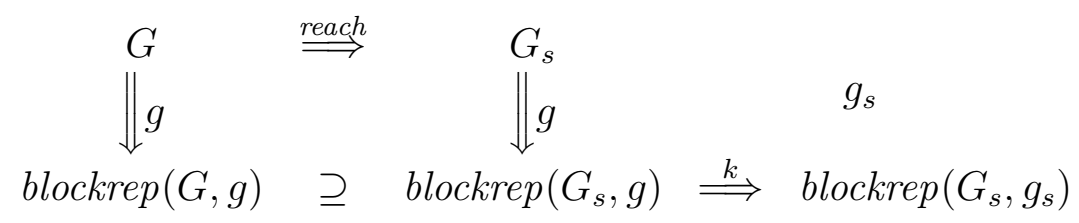

Here the double arrows indicate that the respective functions are of second order, since their arguments, viz. pseudo-graphs and states, are mappings themselves. 


\section{Determining the Reachable Subgraph}

\subsection{A First Recursive Solution}

Let pgraph be the set of pseudo-graphs and nodeset be the set of finite subsets of node. The specification of the reachability problem now reads

funct reach $\equiv$ (pgraph $G$, nodeset $s: s \subseteq \downarrow G$ )pgraph :

$G \mid$ rnset $_{G}(s)$.

From the specification of rnset we derive immediately the property

Lemma 5.1.1

$\operatorname{rnset}_{G}(s \cup t) \equiv \operatorname{rnset}_{G}(s) \cup \operatorname{rnset}_{G}(t)$ for arbitrary $s, t \in$ nodeset.

To derive a recursive algorithm for reach, let a pseudo-graph $G$ and a node set $s \subseteq \downarrow G$ be given. If $s \equiv \emptyset$, then

$$
G \mid \text { rnset }_{G}(s) \equiv G \mid \emptyset \equiv \emptyset .
$$

If $s \not \equiv \emptyset$, we may choose an arbitrary node $z \in s$. Then we have

\section{Lemma 5.1.2}

(1) $\operatorname{ispath}_{G}(p, x, y) \wedge z \notin \operatorname{lead}(p) \equiv \operatorname{ispath}_{G \ominus z}(p, x, y)$,

where $\operatorname{lead}(p) \stackrel{\text { def }}{\equiv}<p[1], \ldots, p[|p|-1]>$.

(2) $\exists x \in s: \exists p: \operatorname{ispath}_{G}(p, x, y) \wedge z \in \operatorname{lead}(p) \equiv$

$\exists p: \operatorname{ispath}_{G}(p, z, y)$.

(3) $\exists p: \operatorname{ispath}_{G}(p, z, y) \wedge z \neq y \equiv$

$\exists u \in \operatorname{set}(G(z)) \backslash\{z\}: \exists p_{1}: \operatorname{ispath}_{G}\left(p_{1}, u, y\right) \wedge z \notin \operatorname{set}\left(p_{1}\right)$.

Proof: (1) Holds, because $\downarrow(G \ominus z) \equiv \downarrow G \backslash\{z\}$.

(2) Assume $\operatorname{ispath}_{G}(p, x, y) \wedge z \in \operatorname{lead}(p)$. Let $q$ be the shortest postfix of $p$ that contains $z$. Then $\operatorname{first}(q)=z$ and $\operatorname{last}(q)=y$, so that $i_{\text {spath }}(q, z, y)$. The reverse implication is trivial.

(3) Assume $\operatorname{ispath}_{G}(p, z, y) \wedge z \neq y$. Let $q$ be the shortest postfix of $p$ that contains $z$. Then $\operatorname{first}(q)=z$ and $\operatorname{last}(q)=y$, so that $\operatorname{ispath}_{G}(q, z, y)$. Since $z \neq y$ we have $|q| \geq 2$ and there must be a $u \in \operatorname{set}(G(z))$ with $u=q[2]$. But then $i_{\text {spath }}(\operatorname{rest}(q), u, y)$ and, by construction, $z \notin \operatorname{set}(\operatorname{rest}(q))$ as well as $u \neq z$.

Assume conversely that $\operatorname{ispath}_{G}\left(p_{1}, u, y\right) \wedge z \notin \operatorname{set}\left(p_{1}\right)$ for some $p_{1}$ and $u \in$ $\operatorname{set}(G(z)) \backslash\{z\}$. Then $\operatorname{ispath}_{G}\left(<z>+p_{1}, z, y\right)$ and $z \neq y$.

Now we obtain

$$
\begin{aligned}
& \operatorname{rnset}_{G}(s) \\
\equiv & \left\{y \mid \exists x \in s: \exists p: \operatorname{ispath}_{G}(p, x, y)\right\} \\
\equiv & \left\{y \mid \exists x \in s: \exists p: \text { ispath }_{G}(p, x, y) \wedge z \notin \operatorname{lead}(p)\right\} \cup \\
& \left\{y \mid \exists x \in s: \exists p: \text { ispath }_{G}(p, x, y) \wedge z \in \operatorname{lead}(p)\right\} \\
\equiv & \left\{y \mid \exists x \in s \backslash\{z\}: \exists p: \operatorname{ispath}_{G}(p, x, y) \wedge z \notin \operatorname{lead}(p)\right\} \cup \\
& \left\{y \mid \exists x \in s: \exists p: \text { ispath }_{G}(p, x, y) \wedge z \in \operatorname{lead}(p)\right\} \\
\equiv & (\text { by }(1))
\end{aligned}
$$




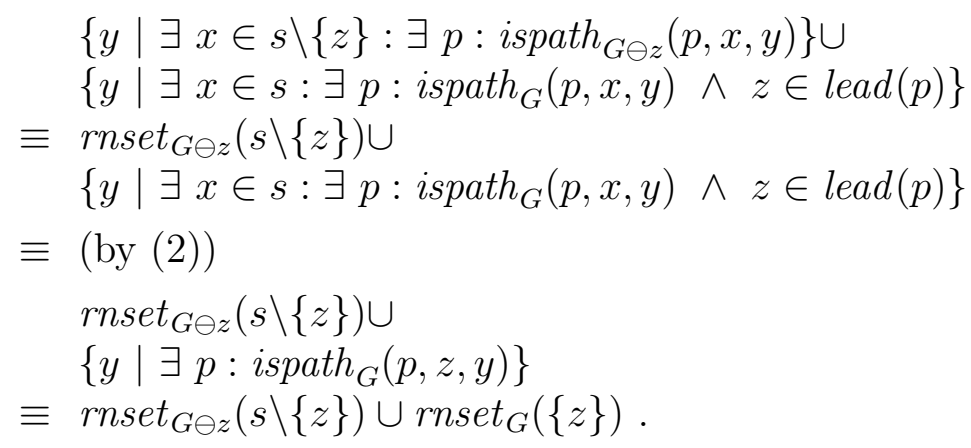

Moreover,

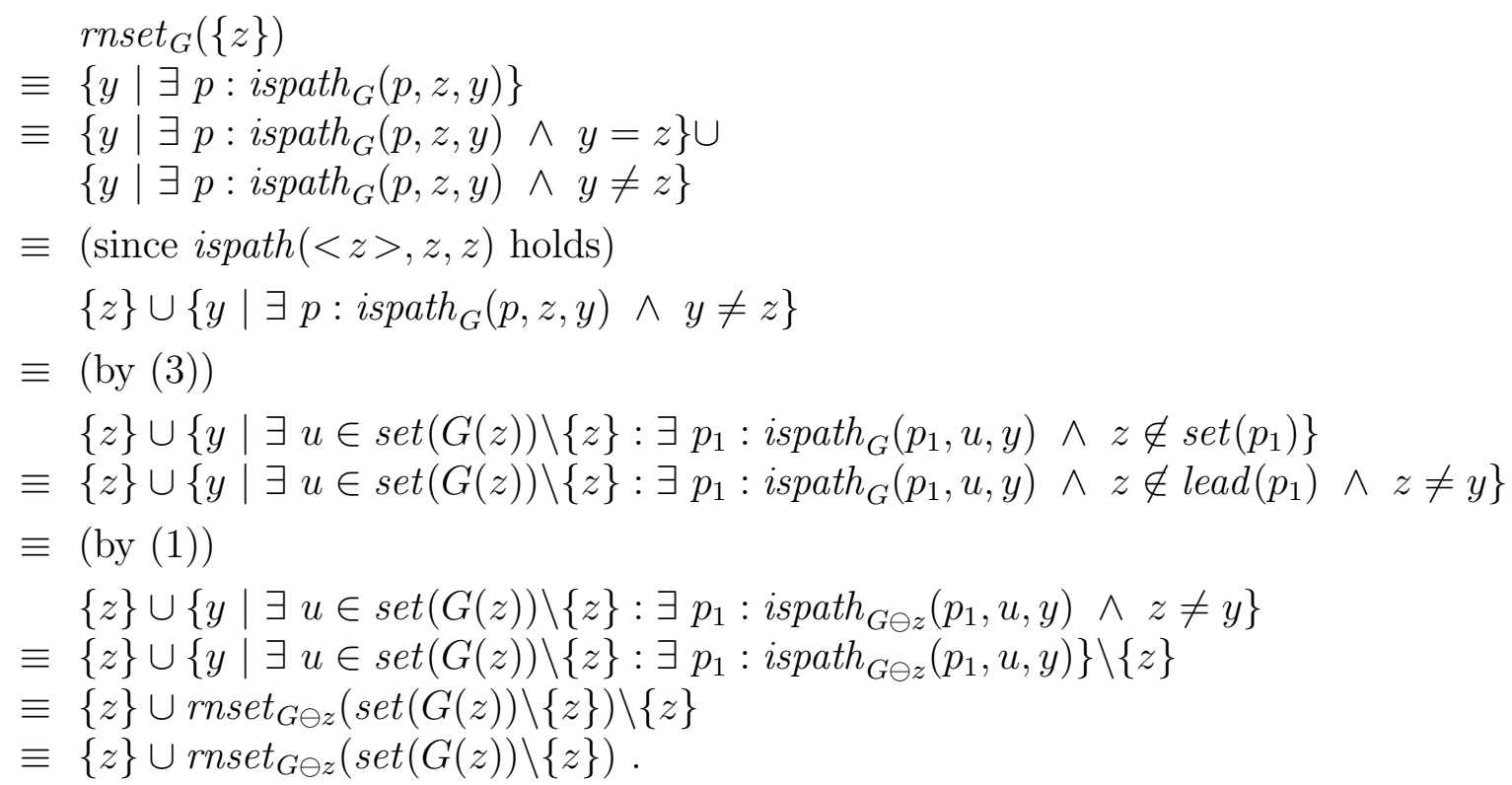

Hence,

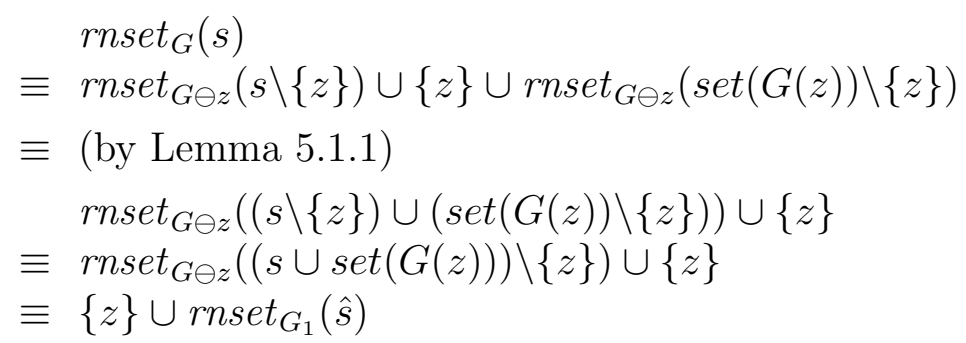

where

$$
\begin{aligned}
G_{1} & \stackrel{\text { def }}{\equiv} G \ominus z, \\
\hat{s} & \stackrel{\text { def }}{\equiv}(s \cup \operatorname{set}(G(z))) \backslash\{z\} .
\end{aligned}
$$

Now we obtain

$$
\begin{aligned}
& \operatorname{reach}(G, s) \\
\equiv & G \mid \operatorname{rnset}_{G}(s) \\
\equiv & G \mid\left(\{z\} \cup \operatorname{rnset}_{G_{1}}(\hat{s})\right) \\
\equiv & \left([z \mapsto G(z)] \cup G_{1}\right) \mid\left(\{z\} \cup \text { rnset }_{G_{1}}(\hat{s})\right) \\
\equiv & {[z \mapsto G(z)]|\{z\} \cup[z \mapsto G(z)]| \text { rnset }_{G_{1}}(\hat{s}) \cup G_{1}\left|\{z\} \cup G_{1}\right| \text { rnset }_{G_{1}}(\hat{s}) } \\
\equiv & {[z \mapsto G(z)] \cup[z \mapsto G(z)] \mid \text { rnset }_{G_{1}}(\hat{s}) \cup \emptyset \cup G_{1} \mid \text { rnset }_{G_{1}}(\hat{s}) } \\
\equiv & \left(\operatorname{since}[z \mapsto G(z)] \mid \text { rnset }_{G_{1}}(\hat{s}) \subseteq[z \mapsto G(z)]\right) \\
& {[z \mapsto G(z)] \cup G_{1} \mid \text { rnset }_{G_{1}}(\hat{s}) . }
\end{aligned}
$$

Next, we observe that we can shrink $\hat{s}$ even further using 


\section{Lemma 5.1.3}

For a pseudo-graph $H$ and a set $s \in$ nodeset we have

$$
H\left|\operatorname{rnset}_{H}(s) \equiv H\right| \operatorname{rnset}_{H}(s \cap \downarrow H) .
$$

Proof: Note first that, by definition of ispath, we have for $z \notin \downarrow H$ that

$$
\operatorname{ispath}(p, z, x) \equiv x=z \wedge p=<z>.
$$

Therefore, if $t \cap \downarrow H \equiv \emptyset$ then $\operatorname{rnset}_{H}(t) \equiv t$. Now,

$$
\begin{aligned}
& \operatorname{rnset}_{H}(s) \\
\equiv & \operatorname{rnset}_{H}((s \cap \downarrow H) \cup(s \backslash \downarrow H)) \\
\equiv & \operatorname{by~Lemma~5.1.1)~} \operatorname{rnset}_{H}(s \cap \downarrow H) \cup \text { rnset }_{H}(s \backslash \downarrow H) \\
\equiv & \operatorname{rnset}_{H}(s \cap \downarrow H) \cup s \backslash \downarrow H
\end{aligned}
$$

and hence

$$
\begin{aligned}
& H \mid \operatorname{rnset}_{H}(s) \\
\equiv & H \mid\left(\operatorname{rnset}_{H}(s \cap \downarrow H) \cup s \backslash \downarrow H\right) \\
\equiv & H\left|\operatorname{rnset}_{H}(s \cap \downarrow H) \cup H\right|(s \backslash \downarrow H) \\
\equiv & H \mid \operatorname{rnset}_{H}(s \cap \downarrow H) \cup \emptyset \\
\equiv & H \mid \operatorname{rnset}_{H}(s \cap \downarrow H) .
\end{aligned}
$$

This gives us finally

$$
\begin{aligned}
& \operatorname{reach}(G, s) \\
\equiv & {[z \mapsto G(z)] \cup G_{1} \mid \text { rnset }_{G_{1}}(\hat{s}) } \\
\equiv & \text { (by the previous } \text { Lemma }) \\
& {[z \mapsto G(z)] \cup G_{1} \mid \text { rnset }_{G_{1}}\left(\hat{s} \cap \downarrow G_{1}\right) } \\
\equiv & {[z \mapsto G(z)] \cup G_{1} \mid \text { rnset }_{G_{1}}\left(((s \cup \operatorname{set}(G(z))) \backslash\{z\}) \cap \downarrow G_{1}\right) } \\
\equiv & {[z \mapsto G(z)] \cup G_{1} \mid \text { rnset }_{G_{1}}\left((s \cup \operatorname{set}(G(z))) \cap \downarrow G_{1}\right) } \\
\equiv & \left(\operatorname{since}(s \cup \operatorname{set}(G(z))) \cap \downarrow G_{1} \subseteq \downarrow G_{1}\right) \\
& {[z \mapsto G(z)] \cup \operatorname{reach}\left(G_{1},\left(s \cup \operatorname{set}(G(z)) \cap \downarrow G_{1}\right)\right.} \\
\equiv & {[z \mapsto G(z)] \cup \operatorname{reach}\left(G_{1}, s_{1}\right) }
\end{aligned}
$$

where

$$
s_{1} \stackrel{\text { def }}{=}(s \cup \operatorname{set}(G(z))) \cap \downarrow G_{1} \text {. }
$$

Defining

$$
\operatorname{elem}(s) \stackrel{\text { def }}{=} s \neq \emptyset \triangleright \text { some node } x: x \in s,
$$

we obtain from this the following recursive version for reach :

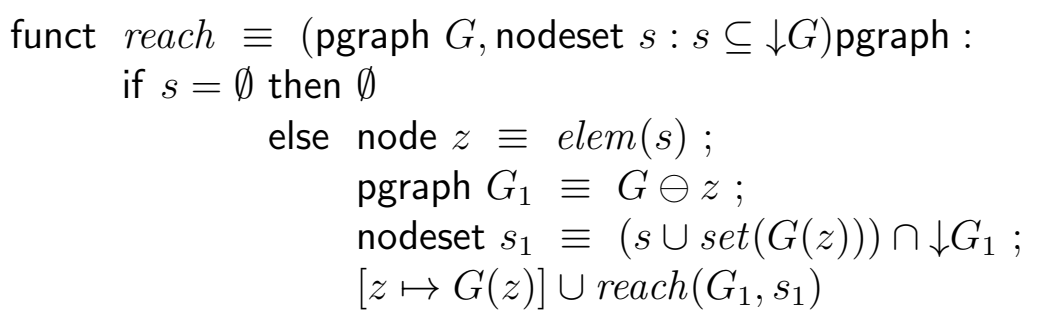


Termination is guaranteed, since $\left|\downarrow G_{1}\right|<|\downarrow G|$. The restriction of reach to proper graphs yields a solution to the original subgraph problem, viz. $G_{s} \equiv \operatorname{reach}(G, s)$ for every $s \in$ nodeset and every graph $G$.

\subsection{Improvement of the Solution}

Since map union is associative, we may by a standard method embed reach into a tail-recursive function reach1:

$$
\operatorname{reach}(G, s) \equiv \operatorname{reach} 1(\emptyset, G, s)
$$

where

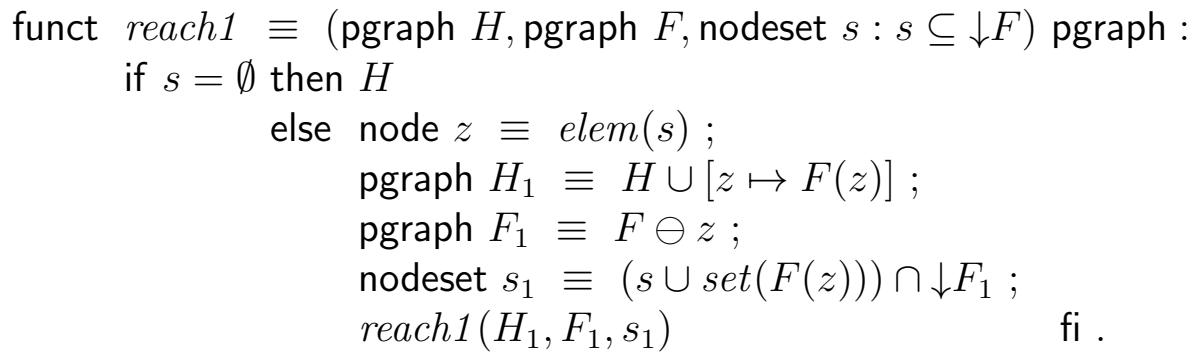

In a second step we eliminate the parameter $F$ from the recursion: Obviously the property

$$
P_{G}(H, F) \stackrel{\text { def }}{\Leftrightarrow} F=G \backslash H
$$

is an invariant of reach1 (i.e., $\left.P_{G}(H, F) \Rightarrow P_{G}\left(H_{1}, F_{1}\right)\right)$, and $P_{G}(\emptyset, G)$ holds. Therefore we may replace $F$ by $G \backslash H$ in reach1. Moreover, $s \subseteq \downarrow F \subseteq \downarrow G \wedge F|s=G| s$ is an additional invariant for the call $\operatorname{reach} 1(\emptyset, G, s)$; hence it follows that

$$
\begin{aligned}
& s_{1} \\
\equiv & (s \cup \operatorname{set}(F(z))) \cap \downarrow F_{1} \\
\equiv & (s \cup \operatorname{set}(G(z))) \cap\left(\downarrow G \backslash \downarrow H_{1}\right) \\
\equiv & (s \cup(\operatorname{set}(G(z))) \cap \downarrow G)) \backslash \downarrow H_{1} .
\end{aligned}
$$

If furthermore $G$ is even a storage graph, we have $\operatorname{set}(G(z)) \subseteq \downarrow G$ and therefore

$$
s_{1} \equiv(s \cup \operatorname{set}(G(z))) \backslash \downarrow H_{1} .
$$

Thus our final algorithm reads

$$
\begin{aligned}
& \text { funct reach } \equiv \text { (pgraph } G \text {, nodeset } s: s \subseteq \downarrow G) \text { pgraph : } \\
& \text { reach } 2(\emptyset, s) \\
& \text { where } \\
& \text { funct reach } 2 \equiv \text { (pgraph } H \text {, nodeset } s: s \subseteq \downarrow G) \text { pgraph : } \\
& \text { if } s=\emptyset \text { then } H \\
& \text { else node } z \equiv \operatorname{elem}(s) \text {; } \\
& \text { pgraph } H_{1} \equiv H \cup[z \mapsto G(z)] \text {; } \\
& \text { nodeset } s_{1} \equiv(s \cup \operatorname{set}(G(z))) \backslash \downarrow H_{1} \text {; } \\
& \text { reach2 }\left(H_{1}, s_{1}\right)
\end{aligned}
$$

This version corresponds to algorithm (7) derived in [Berghammer et al. 87] which computes the set $\downarrow G_{s}$ of nodes reachable from $s$ (replace pgraph by nodeset, $[z \mapsto G(z)]$ by $z$, and $\downarrow H_{1}$ by $H_{1}$ ). 


\section{Copying Pointer Structures}

\subsection{Statement of the Problem}

We now consider the task of copying a state to another part of a memory. Let $\mathcal{M}=($ cell, $\square$ ) be a memory and let $m, n$ be states of $\mathcal{M}$ such that $\square \in \operatorname{set}(m) \cap \operatorname{set}(n)$. We call $n$ a copy of $m$ if there is a total bijection $k: \operatorname{set}(m) \longrightarrow \operatorname{set}(n)$ such that $k(\square) \equiv \square$ and the following diagram commutes:

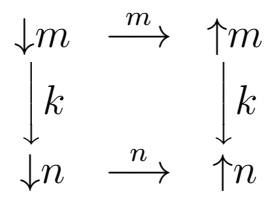

This means that $k \circ m \equiv n \circ k$ and, since $k$ is bijective, that $n \equiv k \circ m \circ k^{-1}$. Hence, given $k$, we can compute $n$ from $m$ in two passes: First we form $k \circ m$ which means that the cell contents as given by $m$ are updated to contain the corresponding addresses of the copy ("pointer relocation pass"); then we compose with $k^{-1}$ which means the actual transport of the new contents to the new locations ("copying pass").

\subsection{Copying Pass}

Given $p \equiv k \circ m$, the copying pass is easily performed. First, by totality of $k$, we have $\downarrow p \equiv$ $\downarrow m(\subseteq \downarrow k)$. Now

$$
\begin{aligned}
& p \circ k^{-1} \\
\equiv & (\text { by Lemma 2.3.3) } \\
& p \circ \bigcup_{x \in \downarrow k}[k(x) \mapsto x] \\
\equiv & (\text { by Lemma 2.3.3) } \\
& \bigcup_{x \in \downarrow k} p \circ[k(x) \mapsto x] \\
\equiv & \bigcup_{x \in \downarrow k}[k(x) \mapsto p(x)] \\
\equiv & \bigcup_{x \in \downarrow p}[k(x) \mapsto p(x)],
\end{aligned}
$$

since $[k(x) \mapsto p(x)] \equiv \emptyset$ for $x \in \downarrow k \backslash \downarrow p \equiv \downarrow k \downarrow \downarrow m$. This union can be computed using the algorithm developed in Section 2.3.2. We set

$$
\operatorname{copass}(p, k) \stackrel{\text { def }}{\equiv} \downarrow p \subseteq \downarrow k \triangleright \operatorname{mapunion}(\downarrow p, k, p) .
$$

\subsection{Pointer Relocation}

The more difficult subtask consists in computing the composition $k \circ m$ efficiently. According to Lemma 2.3.3 there are essentially two ways of forming $k \circ \mathrm{m}$ :

1. domain-oriented:

$$
k \circ m \equiv \bigcup_{x \in \downarrow m}[x \mapsto k(m(x))]
$$


If we look at the union as a loop, this way of forming $k \circ m$ needs an explicit representation of $k$, since the same value of $k$ may be needed repeatedly at irregular intervals.

2. range-oriented:

$$
k \circ m \equiv \bigcup_{z \in \uparrow m}[(z \downarrow m) \mapsto k(z)]
$$

For evaluating this by a loop we only need one value of $k$ at a time to process a whole subset of $\downarrow m$. Hence we can avoid explicit representation of the complete $k$, which is particularly important in garbage collection, where storage is almost exhausted. Moreover, the repeated lookups are avoided and thus also time-efficiency is improved. Of course, this is only interesting if $m$ is highly non-injective so that the inverse images $z \downarrow m$ are large. However, for block representations especially of dense graphs just this is the case.

We follow now the range-oriented variant. We need a way of representing the component maps $[z \downarrow m \mapsto y]$ suitably. For this we use an idea that is presented e.g. in [Dewar, McCann 77]: All elements of $s$ are chained into a linked list; then $[s \mapsto y]$ can be formed as

$$
\bigcup_{x \in s}[x \mapsto y]
$$

following the chain.

\subsubsection{Chains}

In this section we list a number of basic properties of singly linked lists; proofs not stated here can be found in [Möller 90].

Since a partial map $m$ : cell $\longrightarrow$ cell can be thought of as representing a graph of maximal outdegree 1, we may represent linked lists again by maps. The idea of following a chain starting from a point $x$ in a graph of maximal outdegree 1 , represented by $m:$ cell $\longrightarrow$ cell, is captured by considering $m$ as a relation and passing to its transitive closure $m^{+}:$For $x, y \in \operatorname{set}(m)$,

$$
x m^{+} y \stackrel{\text { def }}{\Leftrightarrow} y \in \bigcup_{i \in \mathbb{N} \backslash\{0\}} x \uparrow m^{i}
$$

where

$$
\begin{aligned}
& m^{0} \stackrel{\text { def }}{\equiv} i d_{s e t(m)} \\
& m^{i+1} \stackrel{\text { def }}{\equiv} m \circ m^{i} .
\end{aligned}
$$

Hence $x m^{+} y$ holds iff $y$ can be reached from $x$ following the links of $m$ (at least once). We also need the reflexive transitive closure $m^{*}$ of $m$ given by

$$
x m^{*} y \stackrel{\text { def }}{\Leftrightarrow} y \in \bigcup_{i \in \mathbb{N}} x \uparrow m^{i} .
$$

We call a state $m$ a chain, if $m^{+}$is a linear strict-order on $\operatorname{set}(m)$, i.e., iff the predicate $i s c h a i n(m)$ holds where

$$
\begin{array}{rlll}
\operatorname{ischain}(m) \stackrel{\text { def }}{\Leftrightarrow} & \forall x, y, z \in \operatorname{set}(m): & \\
& \neg x m^{+} x & & \text { (irreflexivity) } \\
& \wedge & x \neq \equiv y \Rightarrow\left(x m^{+} y \vee y m^{+} x\right) & \text { (linearity). }
\end{array}
$$


Irreflexivity excludes the existence of cycles within the list, whereas linearity implies that the list is connected, i.e., that, given two distinct cells in the list, one of them can be reached from the other following the links of the list. The definition implies that a chain is injective and thus that each cell in a chain is referred to by at most one cell. Note also that $\emptyset$ is a chain. $m$ is a finite chain if $m$ is both a chain and a finite set of pairs.

\section{Lemma 6.3.1}

1. For a map $[x \mapsto y]$ we have $[x \mapsto y]^{+} \equiv[x \mapsto y]$, i.e., all such maps are transitive.

2. $[x \mapsto y]$ is a chain iff $x \not \equiv y$.

Proof: $\quad$ 1. $[x \mapsto y] \circ[x \mapsto y] \equiv$ if $x=y$ then $[x \mapsto x]$ else $\emptyset$ fi. Now a straightforward induction shows that for all $i \geq 2$ we have $[x \mapsto y]^{i} \equiv$ if $x=y$ then $[x \mapsto x]$ else $\emptyset$ fi.

2. is immediate from 1 .

The following lemma states a property that is very useful for treating combinations of chains:

\section{Lemma 6.3.2}

1. Let $m$ and $n$ be maps such that $\uparrow n \cap \downarrow m \equiv \emptyset$. Then

$$
(m \cup n)^{+} \equiv m^{+} \cup m^{+} n^{+} \cup n^{+},
$$

where $m^{+} n^{+}$is the relational product of $m^{+}$and $n^{+}$(in diagrammatic order).

2. Let $\left(m_{i}\right)_{i \in I}$ be a family of maps such that $\downarrow m_{i} \cap \uparrow m_{j} \equiv \emptyset$ for $i \not \equiv j$. Then

$$
\begin{aligned}
& \left(\bigcup_{i \in I} m_{i}\right)^{*} \equiv \bigcup_{i \in I} m_{i}^{*} \\
& \left(\bigcup_{i \in I} m_{i}\right)^{+} \equiv \bigcup_{i \in I} m_{i}^{+}
\end{aligned}
$$

Proof: $\quad$ 1. From $\uparrow n \cap \downarrow m \equiv \emptyset$ it follows that $n m \equiv \emptyset$. Now a straightforward induction shows

$$
(m \cup n)^{i} \equiv \bigcup_{k=0}^{i} m^{i-k} n^{k}
$$

for $i>1$. From this the claim is immediate.

2. Because of $\downarrow m_{i} \cap \uparrow m_{j} \equiv \emptyset$ we have $m_{i} m_{j} \equiv m_{j} m_{i} \equiv \emptyset$ for $i \not \equiv j$. Hence $\left(\bigcup_{i \in I} m_{i}\right)^{k}$ $\equiv \bigcup_{i \in I} m_{i}^{k}$ for all $k$ and the claim is immediate.

We now restrict our attention to finite chains.

\section{Lemma 6.3.3}


Let $m \not \equiv \emptyset$ be a finite chain. Then

1. $\operatorname{set}(m)$ contains a least element $\operatorname{first}(m)$ and a greatest element last $(m)$ w.r.t. $m^{+}$.

2. $\uparrow m \subseteq \downarrow m \cup\{\operatorname{last}(m)\}$ and $\downarrow m \subseteq \uparrow m \cup\{\operatorname{first}(m)\}$.

3. $\downarrow m \equiv \operatorname{set}(m) \backslash\{\operatorname{last}(m)\}$ and $\uparrow m \equiv \operatorname{set}(m) \backslash\{\operatorname{first}(m)\}$.

4. $\downarrow m \backslash \uparrow m \equiv\{\operatorname{first}(m)\}$ and $\uparrow m \backslash \downarrow m \equiv\{\operatorname{last}(m)\}$.

5. $\downarrow m \backslash\{\operatorname{first}(m)\} \equiv \uparrow m \backslash\{\operatorname{last}(m)\}$.

We set, for well-founded $m \not \equiv \emptyset$

$$
\begin{aligned}
& \operatorname{rest}(m) \stackrel{\text { def }}{\equiv} \text { if } m=\emptyset \text { then } \emptyset \text { else } m \ominus \operatorname{first}(m) \mathrm{fi}, \\
& \operatorname{inner}(m) \stackrel{\text { def }}{\equiv} \downarrow m \backslash\{\operatorname{first}(m)\} \equiv \uparrow m \backslash\{\operatorname{last}(m)\} .
\end{aligned}
$$

Note that rest is total whereas inner is partial.

\section{Lemma 6.3.4}

Let $m \not \equiv \emptyset$ be a finite chain. Then

1. $m \equiv[\operatorname{first}(m) \mapsto m(\operatorname{first}(m))] \cup \operatorname{rest}(m)$.

2. $\operatorname{rest}(m) \not \equiv \emptyset \Rightarrow \operatorname{first}(\operatorname{rest}(m)) \equiv m(\operatorname{first}(m))$.

3. If $m$ is also anchored, $\operatorname{rest}(m) \equiv \emptyset \Leftrightarrow m(\operatorname{first}(m)) \equiv \square$.

\subsubsection{Chained Representation of Sets}

It would be attractive to call a chain $m$ a representation of $\operatorname{set}(m)$. However, we have

\section{Lemma 6.3.5}

Let $m \not \equiv \emptyset$ be chain. Then $|\operatorname{set}(m)| \geq 2$.

Proof: By definition, $|\operatorname{set}(m)| \geq|\downarrow m|$. Hence, if $|\downarrow m| \geq 2$ we are finished. Otherwise, $|\downarrow m|=1$, i.e., $m \equiv[x \mapsto y]$ for some $x, y \in M$ and $\operatorname{set}(m) \equiv\{x, y\}$. However, since $m$ is irreflexive, we have $x \not \equiv y$ and thus $|\operatorname{set}(m)| \equiv 2$.

According to this lemma, then sets of cardinality 1 would not be representable. To remedy this, we use the distinguished cell $\square$ as a chain terminator and consider all other elements in chains as the elements of the represented sets. Therefore we define the predicate

$$
\begin{aligned}
\operatorname{isanchored}(m) \stackrel{\text { def }}{\Leftrightarrow} & i \operatorname{schain}(m) \wedge \\
& (m \equiv \emptyset \vee \operatorname{last}(m) \equiv \square) .
\end{aligned}
$$

Thus, a nonempty chain is anchored iff it is terminated by $\square$. An anchored chain $m$ represents the set $\downarrow m$. A corresponding representation function is

funct chainrep $\equiv$ (cellset $s)$ state :

some state $m$ : isanchored $(m) \wedge \downarrow m=s$.

We want to develop an incrementation function for extending a set representation to a representation of a larger set. To deal in a satisfactory way with the non-determinacy involved, we need an 
auxiliary notion concerning non-determinacy. Let $E$ be a possibly non-determinate expression. An atom of $E$ is a determinate descendant of $E$. From this definition it is immediate that $E_{1} \subseteq E_{2}$ iff every atom of $E_{1}$ is also an atom of $E_{2}$; likewise, $E_{1} \equiv E_{2}$ iff $E_{1}$ and $E_{2}$ have the same sets of atoms.

Let now $s$ be a set of cells, $m$ be an atom of chainrep $(s)$, and $x \notin s \cup\{\square\}$ be a cell. We want to extend $m$ to an atom $n$ of chainrep $(s \cup\{x\})$. Since this requirement implies $\downarrow n=\downarrow m \cup\{x\}$, we try to set $n \equiv m \cup[x \mapsto y]$ for some $y \in M$. The union is well-defined since $x \notin \downarrow m$ by the assumption. We now have to choose $y$ in such a way that $n$ becomes an anchored chain. First, we compute the transitive closure of $n$ :

$$
\begin{aligned}
& n^{+} \\
\equiv & (m \cup[x \mapsto y])^{+} \\
\equiv & (\text { by Lemma } 6.3 .2) \\
& {[x \mapsto y]^{+} \cup[x \mapsto y]^{+} m^{+} \cup m^{+} } \\
\equiv & (\text { by Lemma 6.3.1) } \\
& {[x \mapsto y] \cup[x \mapsto y] m^{+} \cup m^{+} } \\
\equiv & (*) .
\end{aligned}
$$

Hence isanchored $(n)$ implies $x \not \equiv y$ by irreflexivity. Next we compute first $(n)$. By Lemma 6.3.3,

$$
\begin{aligned}
& \{\operatorname{first}(n)\} \\
\equiv & \downarrow n \backslash \uparrow n \\
\equiv & (\downarrow m \cup\{x\}) \backslash(\uparrow m \cup\{y\}) \\
\equiv & (\downarrow m \backslash \uparrow m \backslash\{y\}) \cup(\{x\} \backslash \uparrow m \backslash\{y\}) \\
\equiv & (\downarrow m \backslash \uparrow m \backslash\{y\}) \cup(\{x\} \backslash\{y\} \backslash \uparrow m) \\
\equiv & (\downarrow m \backslash \uparrow m \backslash\{y\}) \cup(\{x\} \backslash \uparrow m) \\
\equiv & (* *) .
\end{aligned}
$$

Since $x \notin \downarrow m \cup\{\square\} \supseteq \uparrow m$, we have $\{x\} \backslash \uparrow m \equiv\{x\}$. Hence

$$
(* *) \equiv(\downarrow m \backslash \uparrow m \backslash\{y\}) \cup\{x\}
$$

and thus $x \in\{\operatorname{first}(n)\}$, i.e. $x \equiv \operatorname{first}(n)$. Now it follows that $\downarrow m \backslash \uparrow m \backslash\{y\} \subseteq\{x\}$ and hence $\downarrow m \backslash \uparrow m \backslash\{y\} \subseteq \downarrow m \cap\{x\} \equiv \emptyset$, i.e., $\downarrow m \backslash \uparrow m \backslash\{y\} \equiv \emptyset$. If $m \equiv \emptyset$ this holds trivially; however, if $m \not \equiv \emptyset$ this is equivalent to $\{f \operatorname{irst}(m)\} \backslash\{y\} \equiv \emptyset$ and thus also to $y \equiv \operatorname{first}(m)$. So we only need to find $y$ in the case $m \equiv \emptyset$. But by isanchored $(n)$ we know last $(n) \equiv \square$. We calculate

$$
\begin{aligned}
& \{\operatorname{last}(n)\} \\
\equiv & \uparrow n \backslash \downarrow n \\
\equiv & (\uparrow m \cup\{y\}) \backslash(\downarrow m \cup\{x\}) \\
\equiv & \{y\} \backslash\{x\} \\
\equiv & \{y\},
\end{aligned}
$$

and thus $y \equiv \square$. Therefore we define

$$
\operatorname{init}(m) \stackrel{\text { def }}{=} \text { if } m=\emptyset \text { then } \square \text { else } \operatorname{first}(m) \mathrm{fi} .
$$

If we can now show isanchored $(n)$ for

$$
n \stackrel{\text { def }}{\Leftrightarrow} m \cup[x \mapsto \operatorname{init}(m)],
$$

we have found an atom of chainrep $(s \cup\{x\})$. 
From $(*)$ we obtain

$$
\begin{aligned}
u n^{+} v \Leftrightarrow & (u \equiv x \wedge v \equiv y) \vee \\
& \left(u \equiv x \wedge y m^{+} v\right) \vee \\
& \left(u m^{+} v\right) \\
\Leftrightarrow \quad & (u \equiv x \wedge v \in \operatorname{set}(m)) \vee \\
& \left(u m^{+} v\right) .
\end{aligned}
$$

Now we show that $n^{+}$is a linear strict-order. Let $u, v, w \in \operatorname{set}(n)$.

(Irreflexivity) Assume $x n^{+} x$. Then $x \in \operatorname{set}(m) \equiv \downarrow m \cup\{\square\}$, a contradiction to precond $(x, m)$. For $u \not \equiv x, u n^{+} u$ would mean $u \in \downarrow m \wedge u m^{+} u$, contradicting ischain $(m)$ and thus again precond $(x, m)$.

(Linearity) Let $u \not \equiv v$. If $u \equiv x$ then $v \in \operatorname{set}(m)$ and thus $u n^{+} v$. Symmetrically, $v n^{+} u$ if $v \equiv x$. Otherwise, $u, v \in \operatorname{set}(m)$ and therefore $u m^{+} v$ or $v m^{+} u$ by linearity of $m$. But then also $u n^{+} v$ or $v n^{+} u$, resp.

Finally we show that $\operatorname{last}(n) \equiv \square$. If $m \equiv \emptyset$ this is immediate. Otherwise, since $x \equiv \operatorname{first}(n)$, we have $x n^{+} \operatorname{last}(m)$ and therefore $\operatorname{last}(n) \equiv \operatorname{last}(m) \equiv \square$ by isanchored $(m)$.

If we now define

funct $\operatorname{prefix} \equiv($ cell $x$, state $m: \operatorname{isanchored}(m) \wedge x \notin \operatorname{set}(m))$ cell :

$$
[x \mapsto \operatorname{init}(m)] \cup m,
$$

the results of our development can be restated as

\section{Lemma 6.3.6}

1. If $m$ is an atom of chainrep(s) and $x \notin s \cup\{\square\}$, then prefix $(x, m)$ is an atom of chainrep $(s \cup\{x\})$.

2. $\operatorname{first}(\operatorname{prefix}(x, m)) \equiv x \wedge \operatorname{rest}(\operatorname{prefix}(x, m)) \equiv m$.

At this point, it is also interesting to note that

\section{Lemma 6.3.7}

If $m \not \equiv \emptyset$ is an anchored chain, we have prefix $(\operatorname{first}(m), \operatorname{rest}(m)) \equiv m$.

Proof:

$$
\begin{aligned}
& \operatorname{prefix}(\operatorname{first}(m), \operatorname{rest}(m)) \\
& \equiv {[\operatorname{first}(m) \mapsto \operatorname{init}(\operatorname{rest}(m))] \cup \operatorname{rest}(m) } \\
& \equiv \text { if } \operatorname{rest}(m)=\emptyset \text { then }[\operatorname{first}(m) \mapsto \square] \\
& \text { else }[\operatorname{first}(m) \mapsto \operatorname{first}(\operatorname{rest}(m))] \cup \operatorname{rest}(m) \quad \mathrm{fi} \\
& \equiv(\operatorname{by} \operatorname{isanchored}(m)) \\
& \text { if } \operatorname{rest}(m)=\emptyset \text { then } m \\
& \text { else }[\operatorname{first}(m) \mapsto \operatorname{first}(\operatorname{rest}(m))] \cup \operatorname{rest}(m) \quad \mathrm{fi} \\
& \equiv(\operatorname{by} \operatorname{Lemma} 6.3 .4) \\
& \text { if } \operatorname{rest}(m)=\emptyset \text { then } m \text { else } m \mathrm{fi} \\
& \equiv m .
\end{aligned}
$$




\subsubsection{Chained Representation of Maps}

Let now $n$ be a map. From the range-oriented decomposition $n \equiv \bigcup_{z \in \uparrow_{n}}[z \downarrow n \mapsto z]$ we obtain the partition $\downarrow n \equiv \bigcup_{z \in \uparrow n} z \downarrow n$. We represent $m$ by a union of chains each of which represents one of the sets $z \downarrow m$; the cell $z$ is prefixed as a header cell to the respective chain. To avoid confusion between these chains we require that

$$
\operatorname{ischainable}(m) \stackrel{\text { def }}{\Leftrightarrow} \downarrow m \cap \uparrow m \equiv \emptyset
$$

holds; otherwise there would be a link from one chain to the beginning of another and the partition would be lost. We now define

$$
\begin{aligned}
& \text { funct } \text { chain } \equiv \text { (state } m \text { : ischainable }(m)) \text { state : } \\
& \bigcup_{z \in \uparrow m} \operatorname{prefix}(z, \text { chainrep }(z \downarrow m))
\end{aligned}
$$

Since the elements of $z \downarrow m$ are the starting points of mutually unconnected chains, they are also sources (in the graph-theoretic sense) of the chained map. We set, for arbitrary state $n$,

$$
\operatorname{src}(n) \stackrel{\text { def }}{=} \downarrow m \backslash \uparrow m ;
$$

this is the set of cells to which no pointer exists in $n$. To distinguish the sublist of a state $n$ that emanates from a given cell $x$, we define

$$
\operatorname{from}(x, n) \stackrel{\text { def }}{=} m\left|\left(\bigcup_{i \in \mathbb{N}} x \uparrow n^{i}\right) \equiv n\right|\left\{y \mid x n^{*} y\right\}
$$

Note that this sublist need not be a chain, since there may be a cycle. Note also, that from $(x, n) \equiv$ $\emptyset$ if $x \notin \downarrow n$. With the help of these notions we can characterize chainings of maps by the following predicate ischaining:

$$
\begin{aligned}
\operatorname{ischaining}(l) & \stackrel{\text { def }}{=} l=\bigcup_{z \in \operatorname{src}(l)} \operatorname{from}(z, l) \\
& \wedge \forall z \in \operatorname{src}(l): i \operatorname{isanchored}(\operatorname{from}(z, l)) \\
& \wedge \forall z_{1}, z_{2} \in \operatorname{src}(l): z_{1} \neq z_{2} \Rightarrow \downarrow \operatorname{from}\left(z_{1}, l\right) \cap \downarrow \operatorname{from}\left(z_{2}, l\right)=\emptyset .
\end{aligned}
$$

Moreover, we can define the inverse operation to chaining:

$$
\operatorname{unchain}(l) \stackrel{\text { def }}{=} i \operatorname{schaining}(l) \triangleright \bigcup_{z \in \operatorname{src}(l)}[\operatorname{inner}(\operatorname{from}(z, l)) \mapsto z] \text {. }
$$

\section{Lemma 6.3.8}

Let $l$ be an atom of $\operatorname{chain}(m)$. Then $\operatorname{unchain}(l) \equiv m$.

Proof: By definition, $l \equiv \bigcup_{z \in \uparrow m} l_{z}$ where each $l_{z}$ is an atom of prefix $(z$, chainrep $(z \downarrow m))$. Since $z \in \uparrow m$ we have $\downarrow l_{z} \equiv\{z\} \cup z \downarrow m$ and $\uparrow l_{z} \equiv z \downarrow m \cup\{\square\}$. Therefore, $\downarrow l_{z} \cap \uparrow l_{y} \equiv \emptyset$ for $z \not \equiv y$. Hence, for $z \in \downarrow m$, we have $z l^{*} x \Leftrightarrow z l_{z}^{*} x$. Moreover, by Lemma 6.3.2, $l^{*} \equiv \bigcup_{z \in \uparrow m} l_{z}^{*}$. This gives 


$$
\begin{aligned}
& \operatorname{from}(z, l) \\
\equiv & l \mid\left\{x: z l^{*} x\right\} \\
\equiv & l \mid\left\{x: z l_{z}^{*} x\right\} \\
\equiv & l_{z} \mid\left\{x: z l_{z}^{*} x\right\} \\
\equiv & \operatorname{from}\left(z, l_{z}\right) \\
\equiv & l_{z} .
\end{aligned}
$$

Moreover,

$$
\begin{aligned}
& \operatorname{inner}\left(l_{z}\right) \\
\equiv & \downarrow l_{z} \backslash\left\{\text { first }\left(l_{z}\right)\right\} \\
\equiv & (\{z\} \cup \downarrow \text { chainrep }(z \downarrow m)) \backslash\{z\} \\
\equiv & \downarrow \text { chainrep }(z \downarrow m) \\
\equiv & z \downarrow m .
\end{aligned}
$$

Now the claim is immediate from Lemma 2.3.3.

As in the case of set representations, we want to develop an incrementation function for extending a chained representation of a map into one of a larger map. Let therefore $m, n$ be maps and $x \not \equiv \square$ be a cell such that $x \notin \downarrow m$ as well as $n \equiv m \cup[x \mapsto y]$ for some $y$ and ischainable $(n)$ hold. Given a chaining of $m$, we then want to extend it to a chaining of $n$. First we calculate

$$
\begin{aligned}
& \text { ischainable }(n) \\
\Leftrightarrow & \downarrow(m \cup[x \mapsto y]) \cap \uparrow(m \cup[x \mapsto y]) \equiv \emptyset \\
\Leftrightarrow & (\downarrow m \cup\{x\}) \cap(\uparrow m \cup\{y\}) \equiv \emptyset \\
\Leftrightarrow & (\downarrow m \cap \uparrow m) \cup(\downarrow m \cap\{y\}) \cup(\{x\} \cap \uparrow m) \cup(\{x\} \cap\{y\}) \equiv \emptyset \\
\Leftrightarrow & \downarrow m \cap \uparrow m \equiv \emptyset \wedge \downarrow m \cap\{y\} \equiv \emptyset \wedge\{x\} \cap \uparrow m \equiv \emptyset \wedge\{x\} \cap\{y\} \equiv \emptyset \\
\Leftrightarrow & \text { ischainable }(m) \wedge y \notin \downarrow m \wedge x \notin \uparrow m \wedge x \not \equiv y .
\end{aligned}
$$

Let now $l$ be an atom of $\operatorname{chain}(m)$. This means that $l \equiv \bigcup_{z \in \uparrow m} l_{z}$ where each $l_{z}$ is an atom of the respective $\operatorname{prefix}(z$, chainrep $(z \downarrow m))$. Hence rest $\left(l_{z}\right)$ is an atom of chainrep $(z \downarrow m)$.

We have

$$
\downarrow l \equiv \bigcup_{z \in \uparrow m}(\{z\} \cup z \downarrow m)
$$

and hence $x \notin \downarrow l$.

Consider now an $u \in \uparrow n$. To achieve a more uniform calculation we set $l_{u} \stackrel{\text { def }}{=} \emptyset$ if $u \notin \uparrow m$. Then $\operatorname{rest}\left(l_{u}\right) \equiv \emptyset \equiv$ chainrep $(u \downarrow m)$, and hence $\operatorname{rest}\left(l_{u}\right) \subseteq$ chainrep $(u \downarrow m)$ also in this case.

Case 1: $u \not \equiv y$. Then $u \downarrow n \equiv u \downarrow m$, and hence $l_{u}$ is an atom of prefix $(u, \operatorname{chainrep}(u \downarrow m))$.

Case 2: $u \equiv y$. Then

$$
\begin{aligned}
& \operatorname{prefix}(u, \text { chainrep }(u \downarrow n)) \\
& \equiv \operatorname{prefix}(u, \text { chainrep }(u \downarrow m \cup\{x\})) \\
& \supseteq \operatorname{prefix}(u, \operatorname{prefix}(x, \operatorname{chainrep}(u \downarrow m))) \\
& \supseteq \operatorname{prefix}\left(u, \operatorname{prefix}\left(x, \operatorname{rest}\left(l_{u}\right)\right)\right) .
\end{aligned}
$$

Hence

$$
\begin{aligned}
& \equiv \bigcup_{z \in \uparrow n}^{\operatorname{chain}(n)} \operatorname{prefix}(z, \text { chainrep }(z \downarrow n)) \\
& \equiv \bigcup_{z \in \uparrow m \cup\{y\}} \operatorname{prefix}(z, \text { chainrep }(z \downarrow n))
\end{aligned}
$$




$$
\begin{aligned}
& \equiv \bigcup_{z \in \uparrow m \backslash\{y\} \cup\{y\}} \operatorname{prefix}(z, \text { chainrep }(z \downarrow n)) \\
& \equiv \bigcup \operatorname{prefix}(z, \operatorname{chainrep}(z \downarrow n)) \cup \operatorname{prefix}(y, \operatorname{chainrep}(y \downarrow n)) \\
& z \in \uparrow m \backslash\{y\} \\
& \supseteq \bigcup l_{z} \cup \operatorname{prefix}\left(y, \operatorname{prefix}\left(x, \operatorname{rest}\left(l_{y}\right)\right)\right) \\
& z \in \uparrow m \backslash\{y\} \\
& \equiv l \backslash l_{y} \cup \operatorname{prefix}\left(y, \operatorname{prefix}\left(x, \operatorname{rest}\left(l_{y}\right)\right)\right) \\
& \equiv l \ominus \downarrow l_{y} \cup \operatorname{prefix}\left(y, \operatorname{prefix}\left(x, \operatorname{rest}\left(l_{y}\right)\right)\right) \\
& \equiv \text { (by Lemma 2.3.4, since } x \notin \downarrow l) \\
& l \nLeftarrow \operatorname{prefix}\left(y, \operatorname{prefix}\left(x, \operatorname{rest}\left(l_{y}\right)\right)\right) \\
& \equiv l \nLeftarrow\left(\left[y \mapsto \operatorname{first}\left(\operatorname{prefix}\left(x, \operatorname{rest}\left(l_{y}\right)\right)\right)\right] \cup \operatorname{prefix}\left(x, \operatorname{rest}\left(l_{y}\right)\right)\right) \\
& \equiv l \nLeftarrow\left([y \mapsto x] \cup\left[x \mapsto \operatorname{init}\left(\operatorname{rest}\left(l_{y}\right)\right)\right] \cup \operatorname{rest}\left(l_{y}\right)\right) \\
& \equiv \text { (by Lemma 2.3.4 (Annihilation), since } \operatorname{rest}\left(l_{y}\right) \subseteq l \text { ) } \\
& l \nLeftarrow\left([y \mapsto x] \cup\left[x \mapsto \operatorname{init}\left(\operatorname{rest}\left(l_{y}\right)\right)\right]\right) \\
& \equiv \text { if } y \in \uparrow m \text { then } l \nLeftarrow\left([y \mapsto x] \cup\left[x \mapsto \operatorname{first}\left(\operatorname{rest}\left(l_{y}\right)\right)\right]\right) \\
& \text { else } l \nLeftarrow([y \mapsto x] \cup[x \mapsto \square]) \text { fi } \\
& \equiv \text { if } y \in \uparrow m \text { then } l \nLeftarrow\left([y \mapsto x] \cup\left[x \mapsto l_{y}(y)\right]\right) \\
& \text { else } l \nLeftarrow([y \mapsto x] \cup[x \mapsto \square]) \text { fi } \\
& \equiv \text { if } y \in \uparrow m \text { then } l \nLeftarrow([y \mapsto x] \cup[x \mapsto l(y)]) \\
& \text { else } l \nLeftarrow([y \mapsto x] \cup[x \mapsto \square]) \text { fi . }
\end{aligned}
$$

Hence we define

$$
\begin{aligned}
& \operatorname{insert}(l, y, x) \\
& \stackrel{\text { def }}{=} \operatorname{ischaining}(l) \wedge x \notin \operatorname{set}(l) \wedge y \notin \operatorname{set}(l) \backslash \operatorname{src}(l) \wedge x \neq y \triangleright \\
& \text { if } y \in \operatorname{src}(l) \text { then } l \nLeftarrow([y \mapsto x] \cup[x \mapsto l(y)]) \\
& \text { else } l \nLeftarrow([y \mapsto x] \cup[x \mapsto \square]) \mathrm{fi} .
\end{aligned}
$$

The results of the above development then are summarized by

\section{Lemma 6.3.9}

Assume ischaining $(l) \wedge x \notin \operatorname{set}(l) \wedge y \notin \operatorname{set}(l) \backslash \operatorname{src}(l) \wedge x \neq y$. Then $\operatorname{insert}(l, y, x)$ is an atom of $\operatorname{chain}($ unchain $(l) \cup[x \mapsto y])$.

\subsubsection{Pointer Relocation Completed}

We are now in a position to describe our efficient algorithm for computing $k \circ m$ : We first construct a chained representation $l \equiv \bigcup_{z \in \uparrow m} l_{z}$ of $m$. Now we define

$$
\text { relocate }(l, k) \stackrel{\text { def }}{=} i \operatorname{schaining}(l) \triangleright k \circ \operatorname{unchain}(l) \text {. }
$$

We have

$$
\begin{aligned}
& \text { relocate }(l, k) \\
& \equiv k \circ \bigcup\left[\operatorname{inner}\left(l_{z}\right) \mapsto z\right] \\
& \equiv \bigcup_{z \in \operatorname{src}(l)}^{z \in \operatorname{src}(l)}\left[\operatorname{inner}\left(l_{z}\right) \mapsto k(z)\right] \text {. }
\end{aligned}
$$

This is the main loop of our algorithm. We now want to develop a more direct version of the inner loops that form the maps $\left[\operatorname{inner}\left(l_{z}\right) \mapsto k(z)\right]$ for $z \in \operatorname{src}(l)$. To this end we define

$$
\text { fibre }(l, x, y) \stackrel{\text { def }}{\equiv} \text { isanchored }(l) \wedge x \in \downarrow l \triangleright[\operatorname{ginner}(l, x) \mapsto y]
$$


where

$$
\operatorname{ginner}(l, x) \stackrel{\text { def }}{=}\left\{z \mid x l^{+} z\right\} \backslash\{\square\} \text {. }
$$

We have

$$
\operatorname{ginner}(l, x) \equiv \text { if } l(x)=\square \text { then } \emptyset \text { else }\{l(x)\} \cup \operatorname{ginner}(l, l(x)) \mathrm{fi}
$$

and hence

$$
\begin{aligned}
& f i b r e(l, x, y) \\
\equiv \text { if } l(x)=\square \text { then }[\operatorname{ginner}(l, x) \mapsto y] & \text { else }[\operatorname{ginner}(l, x) \mapsto y] \mathrm{fi} \\
\equiv \text { if } l(x)=\square \text { then }[\emptyset \mapsto y] & \quad \text { else }[\{l(x)\} \cup \operatorname{ginner}(l, l(x)) \mapsto y] \mathrm{fi} \\
\equiv \text { if } l(x)=\square \text { then } \emptyset & \quad \text { else }[\{l(x)\} \mapsto y] \cup[\operatorname{ginner}(l, l(x)) \mapsto y] \mathrm{fi} \\
\equiv \text { if } l(x)=\square & \text { then } \emptyset \\
& \text { else }[l(x) \mapsto y] \cup \text { fibre }(l, l(x), y) \mathrm{fi} .
\end{aligned}
$$

Hence we have the recursion (termination is obvious)

$$
\begin{aligned}
& \text { funct } \begin{aligned}
\text { fibre } \equiv(\text { state } l, \text { cell } x, y: \text { isanchored }(l) \wedge x \in \downarrow l) \text { state : } \\
\lceil\text { cell } z \equiv l(x) ; \text { if } z=\square \text { then } \emptyset \\
\text { else }[z \mapsto y] \cup f i b r e(l, z, y) \text { fi }\rfloor .
\end{aligned}
\end{aligned}
$$

Finally, we obtain

$$
\begin{aligned}
& \equiv \bigcup_{x \in \operatorname{src}(l)}^{\text {relocate }(l, k)}\left[\operatorname{inner}\left(l_{x}\right) \mapsto k(x)\right] \\
& \equiv \bigcup_{x \in \operatorname{src}(l)} \text { fibre }(l, x, k(x)) .
\end{aligned}
$$

\subsection{Combining Relocation and Copying}

Suppose that unchain $(l) \equiv m$. Then

$$
\begin{aligned}
& k \circ m \circ k^{-1} \\
\equiv & k \circ \operatorname{unchain}(l) \circ k^{-1} \\
\equiv & \operatorname{relocate}(l, k) \circ k^{-1} \\
\equiv & \operatorname{copass}(\operatorname{relocate}(l, k), k) .
\end{aligned}
$$

Therefore we define

$$
\begin{aligned}
\operatorname{copy}(l, k) \stackrel{\text { def }}{=} & i \operatorname{schaining}(l) \wedge \text { isinjective }(k) \wedge \downarrow k=\operatorname{set}(m) \triangleright \\
& \operatorname{copass}(\text { relocate }(l, k), k) .
\end{aligned}
$$

Then the following diagram commutes:

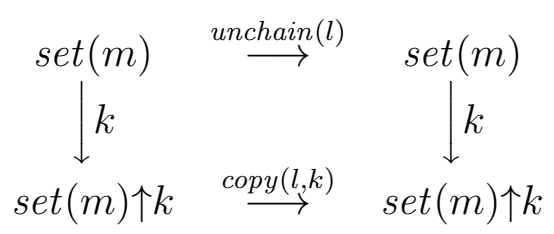

This concludes our treatment of the pointer relocation pass. 


\section{Merging Reachability and Chaining}

\subsection{Statement of the Problem}

In the previous section we have derived an efficient copying algorithm based on a chaining of the state to be copied. In this section we want to integrate the construction of such a representation with the computation of the reachable part.

A central assumption for chainable maps was that their domains should be disjoint from their ranges. Since, however, pseudo-graph states do not have this property, we cannot chain the whole reachable substate, but only its arcs (see 3.2).

More precisely, assume a pseudo-graph $G$, a set $s \subseteq \downarrow G$, and a perfect allocation $g$ of $G$. Define

$$
\begin{array}{lll}
n & \stackrel{\text { def }}{\equiv} & \operatorname{blockrep}(G, g), \\
G_{1} & \stackrel{\text { def }}{\equiv} & \operatorname{reach}(G, s), \\
n_{1} & \stackrel{\text { def }}{\equiv} & \operatorname{blockrep}\left(G_{1}, g\right), \\
t & \stackrel{\text { def }}{\equiv} & s \uparrow g .
\end{array}
$$

We want to compute a chaining of $\operatorname{arcs}\left(n_{1}\right)$. To this end, we shall first compute $\operatorname{arcs}\left(n_{1}\right)$ from $n$ and $t$ by a function sreach and then chain this using a function csreach. Hence sreach and csreach should satisfy the equations

$$
\begin{aligned}
& \operatorname{sreach}(n, t) \equiv \operatorname{arcs}\left(n_{1}\right), \\
& \operatorname{unchain}(\operatorname{csreach}(n, t)) \equiv \operatorname{arcs}\left(n_{1}\right) .
\end{aligned}
$$

In the sequel we shall assume that $G$ is a fragment of a fixed storage-graph $G_{0}$ (see 3.1), i.e., that $G \subseteq G_{0}$. Consequently, $n \subseteq n_{0}$ where $n_{0} \stackrel{\text { def }}{=} \operatorname{blockrep}\left(G_{0}, g\right)$. This will considerably simplify the invariants of the algorithms we shall derive in the sequel.

\subsection{Reachability on Graph Representations}

In Section 5.1 we have derived the following reachability algorithm for pseudo-graphs:

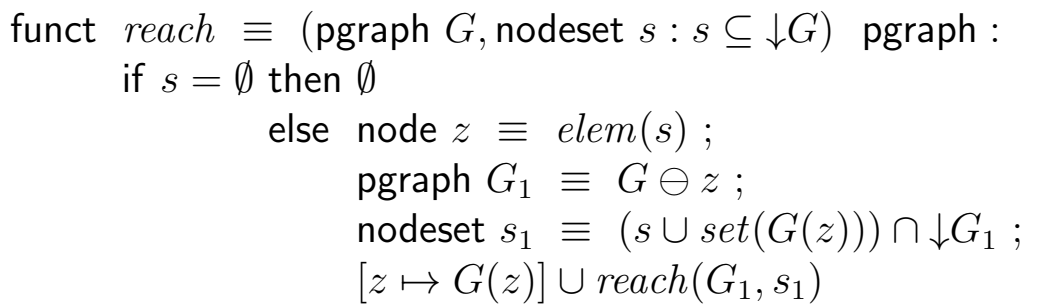

Our first task consists in transforming this into a corresponding algorithm for state representations of pseudo-graphs. Let $g$ be an allocation of a pseudograph $G$. The set $s$ of starting nodes in the pseudo-graph case is now replaced by a set $t$ of starting keys. Thus we define

$$
\begin{aligned}
\text { inreach1 }(G, g, n, t) \stackrel{\text { def }}{\equiv} & n=\operatorname{blockrep}(G, g) \wedge t \subseteq \operatorname{keys}(n) \triangleright \\
& \text { blockrep }(\operatorname{reach}(G, t \downarrow g), g) .
\end{aligned}
$$

Now assume $n \equiv \operatorname{blockrep}(G, g)$ and $\emptyset \not \equiv t \subseteq \operatorname{keys}(n)$. Define

$$
\begin{array}{ll}
x & \stackrel{\text { def }}{\equiv} \text { elem }(t \downarrow g), \\
G_{1} & \stackrel{\text { def }}{\equiv} G \ominus x, \\
s_{1} & \stackrel{\text { def }}{\equiv}(t \downarrow g \cup \operatorname{set}(G(x))) \cap \downarrow G_{1}, \\
n_{1} & \stackrel{\text { def }}{\equiv} \operatorname{blockrep}\left(G_{1}, g\right) .
\end{array}
$$


Then

$$
\begin{aligned}
& \text { inreach1 }(G, g, n, t) \\
\equiv & \operatorname{blockrep}\left([x \mapsto G(x)] \cup \operatorname{reach}\left(G_{1}, s_{1}\right), g\right) \\
\equiv & (\operatorname{by~Lemma~3.2.2)~} \\
& \operatorname{blockrep}([x \mapsto G(x)], g) \cup \text { blockrep }\left(\operatorname{reach}\left(G_{1}, s_{1}\right), g\right) \\
\equiv & \operatorname{block}(x, g) \cup \text { inreach } 1\left(G_{1}, g, n_{1}, s_{1} \uparrow g\right) .
\end{aligned}
$$

Now we calculate

$$
\begin{aligned}
& s_{1} \uparrow g \\
\equiv & \left((t \downarrow g \cup \operatorname{set}(G(x))) \cap \downarrow G_{1}\right) \uparrow g \\
\equiv & (\text { by Lemma 2.3.2, since } g \text { is injective }) \\
& ((t \downarrow g) \uparrow g \cup \operatorname{set}(G(x)) \uparrow g) \cap\left(\downarrow G_{1} \uparrow g\right) \\
\equiv & (t \cup \uparrow(\text { block }(x, g) \ominus g(x))) \cap \text { keys }\left(n_{1}\right) \\
\equiv & (t \cup \uparrow \text { block }(x, g) \backslash\{\square\}) \cap \text { keys }\left(n_{1}\right) \\
\equiv & (t \cup \uparrow \text { block }(x, g)) \cap \text { keys }\left(n_{1}\right) .
\end{aligned}
$$

since $\square \notin$ keys $\left(n_{1}\right)$. Thus we obtain the recursion

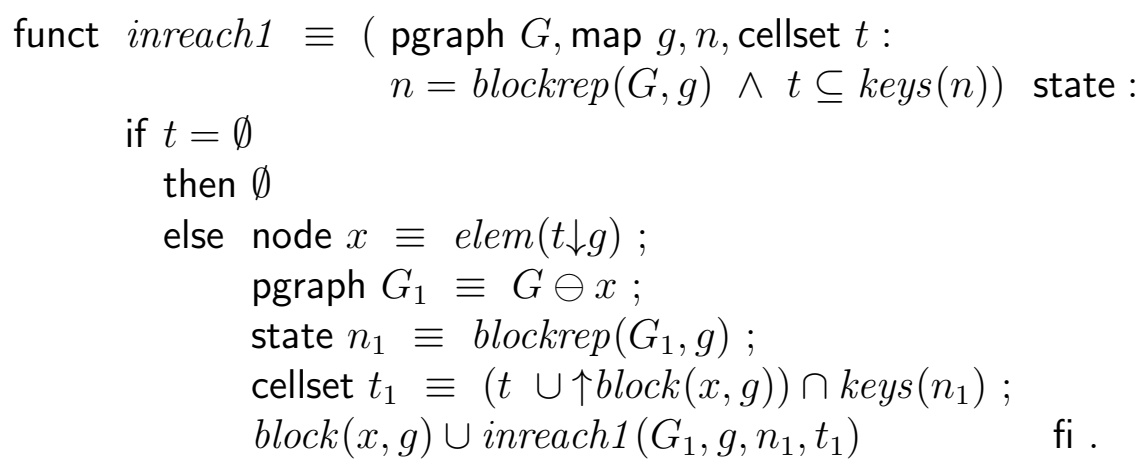

\subsection{Computing the Arcs of the Reachable Substate}

Our next task consists in deriving from inreach1 an algorithm sreach satisfying (SR). An additional requirement for this algorithm is that it should not use a separate parameter for the set $t$ which would occupy a lot of storage space. Everything should be done on the map-parameter $n$ and on some auxiliary cell-parameters.

A first idea how to realize this would be to represent the set $t$ as a chain and to overwrite $n$ with this chain. However, one sees immediately that this is in conflict with the chaining for representing the map which requires a different overwriting of $n$. We shall overcome these difficulties by not storing $t$ itself but (a code of) a set of cells pointing to the elements of $t$ and, when adding block $(x, g)$, by chaining the cells not in their original order but in a different one arising during the traversal of the reachable part.

Let again

$$
\begin{array}{lll}
n & \stackrel{\text { def }}{\equiv} & \operatorname{blockrep}(G, g), \\
G_{1} & \stackrel{\text { def }}{\equiv} & \operatorname{reach}(G, s), \\
n_{1} & \stackrel{\text { def }}{\equiv} & \operatorname{blockrep}\left(G_{1}, g\right), \\
t & \stackrel{\text { def }}{\equiv} & s \uparrow g .
\end{array}
$$

Using the fact that

$$
G_{1} \equiv G \mid s \cup \operatorname{reach}(\tilde{G}, \tilde{s})
$$


where $\tilde{G} \stackrel{\text { def }}{\equiv} G \ominus s$ and $\tilde{s} \stackrel{\text { def }}{=}\left(\bigcup_{x \in s} \operatorname{set}(G(x))\right) \cap \downarrow \tilde{G}$, we calculate

$$
\begin{aligned}
& \operatorname{arcs}\left(n_{1}\right) \\
\equiv & \operatorname{arcs}\left(\operatorname{blockrep}\left(G_{1}, g\right)\right) \\
\equiv & (\operatorname{by~Lemma~3.2.2)~} \\
& \operatorname{arcs}(\operatorname{blockrep}(G \mid s, g)) \cup \operatorname{arcs}(\operatorname{blockrep}(\operatorname{reach}(\tilde{G}, \tilde{s}))) \\
\equiv & \tilde{n} \mid u \cup \operatorname{arcs}(\operatorname{inreach} 1(\tilde{G}, g, m, \tilde{s} \uparrow g))
\end{aligned}
$$

where

$$
\begin{aligned}
\tilde{n} & \stackrel{\text { def }}{\equiv} n \ominus t, \\
u & \stackrel{\text { def }}{\equiv} \operatorname{setfollowers}(n, t) \stackrel{\text { def }}{=} \bigcup_{z \in t} \operatorname{set}(\text { followers }(n, z)) \quad \text { (see 3.2), } \\
m & \stackrel{\text { def }}{\equiv} \operatorname{blockrep}(\tilde{G}, g) .
\end{aligned}
$$

Furthermore,

$$
\tilde{s} \uparrow g \equiv u \uparrow \tilde{n} \cap \operatorname{keys}(\tilde{n})
$$

This suggests the definition

$$
\begin{aligned}
\text { inreach2 }(G, g, n, m, u) \stackrel{\text { def }}{\equiv} & P_{2}(G, g, n, m, u) \triangleright \\
& n \mid u \cup \operatorname{arcs}(\text { inreach1 }(G, g, m, u \uparrow n \cap \operatorname{keys}(n))),
\end{aligned}
$$

where

$$
\begin{aligned}
P_{2}(G, g, n, m, u) & \stackrel{\text { def }}{\equiv} m=\operatorname{blockrep}(G, g) \\
& \wedge n \supseteq m \\
& \wedge \operatorname{keys}(n)=\operatorname{keys}(m) \\
& \wedge u \subseteq \downarrow \operatorname{arcs}(n) \\
& \wedge u \cap \downarrow m=\emptyset \\
& \wedge \operatorname{setfollowers}(n, \operatorname{keys}(n)) \cap u=\emptyset .
\end{aligned}
$$

Using the notation from above, we obtain

$$
\operatorname{arcs}\left(n_{1}\right) \equiv \operatorname{inreach2}(\tilde{G}, g, \tilde{n}, m, u) .
$$

Now we have to develop a recursion for inreach2. This turns out to be the most difficult step in the whole development.

Suppose that $P_{2}(G, g, n, m, u)$ and $u \not \equiv \emptyset$ hold and define

$$
\begin{array}{lcl}
t & \stackrel{\text { def }}{\equiv} & u \uparrow n \cap \operatorname{keys}(n), \\
y & \stackrel{\text { def }}{\equiv} & \operatorname{elem}(u), \\
z & \stackrel{\text { def }}{\equiv} & n(y), \\
n_{1} & \stackrel{\text { def }}{\equiv} & n \ominus y, \\
u_{1} & \stackrel{\text { def }}{\equiv} & u \backslash\{y\}, \\
\tilde{t} & \stackrel{\text { def }}{\equiv} & u_{1} \uparrow n_{1} \cap \operatorname{keys}\left(n_{1}\right) .
\end{array}
$$

First,

$$
\begin{aligned}
& n \mid u \\
\equiv & n \mid\left(\{y\} \cup u_{1}\right) \\
\equiv & n|\{y\} \cup n| u_{1} \\
\equiv & {[y \mapsto z] \cup n_{1} \mid u_{1} . }
\end{aligned}
$$


Moreover, since $y \in \downarrow \operatorname{arcs}(n)$ we have $\operatorname{keys}\left(n_{1}\right) \equiv \operatorname{keys}(n)$. Hence

$$
\begin{aligned}
& t \\
\equiv & \left(u_{1} \cup\{y\}\right) \uparrow n \cap \operatorname{keys}(n) \\
\equiv & \left(u_{1} \uparrow n \cap \operatorname{keys}(n)\right) \cup(\{y\} \uparrow n \cap \operatorname{keys}(n)) \\
\equiv & \left(u_{1} \uparrow n_{1} \cap \operatorname{keys}\left(n_{1}\right)\right) \cup(\{z\} \cap \operatorname{keys}(n)) \\
\equiv & \tilde{t} \cup(\{z\} \cap \operatorname{keys}(n)) .
\end{aligned}
$$

Case 1: $z \notin \operatorname{keys}(n)$, i.e., $(\{z\} \cap \operatorname{keys}(n)) \equiv \emptyset$.

Then $t \equiv \tilde{t}$ and thus

$$
\begin{aligned}
& \text { inreach2 }(G, g, n, m, u) \\
\equiv & n \mid u \cup \operatorname{arcs}(\text { inreach1 }(G, g, m, t)) \\
\equiv & {[y \mapsto z] \cup n_{1} \mid u \cup \operatorname{arcs}(\text { inreach1 }(G, g, m, \tilde{t})) } \\
\equiv & {[y \mapsto z] \cup \text { inreach2 }\left(G, g, n_{1}, m, u_{1}\right) . }
\end{aligned}
$$

Case 2: $z \in \operatorname{keys}(n)$, i.e., $(\{z\} \cap \operatorname{keys}(n)) \equiv\{z\}$.

Then $t \equiv \tilde{t} \cup\{z\}$. Now we specialize the choice of elem $(t \downarrow g)$ in inreach 1 to $g^{-1}(z)$, i.e., we set

$$
\begin{array}{ll}
x & \stackrel{\text { def }}{=} g^{-1}(z), \\
G_{1} & \stackrel{\text { def }}{\equiv} G \ominus x, \\
m_{1} & \stackrel{\text { def }}{\equiv} \\
t_{1} & \stackrel{\text { def }}{\equiv}\left(t \cup \text { lockep }\left(G_{1}, g\right),\right. \\
&
\end{array}
$$

We have keys $\left(m_{1}\right) \equiv \operatorname{keys}(m) \backslash\{z\}$. Hence, setting

$$
n_{2} \stackrel{\text { def }}{\equiv} n_{1} \ominus z
$$

we have

$$
\operatorname{keys}\left(m_{1}\right) \equiv \operatorname{keys}\left(n_{2}\right) \text { and } n_{1}\left|u_{1} \equiv n_{2}\right| u_{1},
$$

since $z \notin \downarrow n_{1} \equiv u_{1} \subseteq \downarrow \operatorname{arcs}(n)$. Moreover,

$$
\begin{aligned}
& \text { inreach2 }(G, g, n, m, u) \\
\equiv & n \mid u \cup \operatorname{arcs}(\text { inreach1 }(G, g, m, t)) \\
\equiv & {[y \mapsto z] \cup n_{1} \mid u_{1} \cup \operatorname{arcs}\left(\operatorname{block}(x, g) \cup \operatorname{inreach} 1\left(G_{1}, g, m_{1}, t_{1}\right)\right) } \\
\equiv & {[y \mapsto z] \cup n_{1} \mid u_{1} \cup \operatorname{arcs}(\operatorname{block}(x, g)) \cup \operatorname{arcs}\left(\text { inreach1 }\left(G_{1}, g, m_{1}, t_{1}\right)\right) } \\
\equiv & {[y \mapsto z] \cup n_{2} \mid u_{1} \cup \operatorname{arcs}(\operatorname{block}(x, g)) \cup \operatorname{arcs}\left(\operatorname{inreach} 1\left(G_{1}, g, m_{1}, t_{1}\right)\right) . }
\end{aligned}
$$

Now we observe that, since $n \supseteq m \equiv \operatorname{blockrep}(G, g)$,

$$
\begin{aligned}
& \operatorname{block}(x, g) \\
\equiv & n \mid(\{g(x)\} \cup \operatorname{set}(\text { followers }(n, g(x)))) \\
\equiv & n \mid(\{z\} \cup \operatorname{set}(\text { followers }(n, z))) .
\end{aligned}
$$

Let therefore

$$
u_{2} \stackrel{\text { def }}{=} \operatorname{set}(\text { followers }(n, z))
$$

Then

$$
\begin{aligned}
& \operatorname{arcs}(\operatorname{block}(x, g)) \\
\equiv & \operatorname{arcs}\left(n \mid\left(\{z\} \cup u_{2}\right)\right) \\
\equiv & \operatorname{arcs}\left(n|\{z\} \cup n| u_{2}\right) \\
\equiv & \operatorname{arcs}\left(n \mid(\{z\}) \cup \operatorname{arcs}\left(n \mid u_{2}\right)\right.
\end{aligned}
$$


$\equiv($ since $z \in \operatorname{keys}(n \mid\{z\})$ and thus $\operatorname{arcs}(n \mid\{z\}) \equiv \emptyset)$ $\operatorname{arcs}\left(n \mid u_{2}\right)$

$\equiv\left(\operatorname{since} u_{2} \cap \operatorname{keys}(n) \equiv \emptyset\right.$ by $\left.n \supseteq \operatorname{block}(x, g)\right)$ $n \mid u_{2}$.

Hence

$$
\begin{aligned}
& {[y \mapsto z] \cup n_{2} \mid u_{1} \cup \operatorname{arcs}(\operatorname{block}(x, g)) } \\
\equiv & {[y \mapsto z] \cup n_{2}\left|u_{1} \cup n\right| u_{2} } \\
\equiv & \left([y \mapsto z] \cup n \mid u_{2}\right) \cup n_{2} \mid u_{1} \\
\equiv & \left([y \mapsto z] \cup n_{2} \mid u_{2}\right) \cup n_{2} \mid u_{1} \\
\equiv & {[y \mapsto z] \cup n_{2} \mid\left(u_{2} \cup u_{1}\right) }
\end{aligned}
$$

If we now can show $\left(u_{1} \cup u_{2}\right) \uparrow n_{2} \cap \operatorname{keys}\left(n_{2}\right) \equiv t_{1}$ and $\left(u_{1} \cup u_{2}\right) \cap \downarrow m_{1} \equiv \emptyset$, we have derived a recursion relation for inreach2. We have

$$
t_{1} \equiv\left(t \cap \operatorname{keys}\left(m_{1}\right)\right) \cup\left(\uparrow \operatorname{block}(x, g) \cap \operatorname{keys}\left(m_{1}\right)\right)
$$

Now,

$$
\begin{aligned}
& t \cap \operatorname{keys}\left(m_{1}\right) \\
\equiv & u \uparrow n \cap \operatorname{keys}(n) \cap \operatorname{keys}\left(m_{1}\right) \\
\equiv & \left(\operatorname{since} m_{1} \subseteq m \subseteq n\right) \\
& u \uparrow n \cap \operatorname{keys}\left(m_{1}\right) \\
\equiv & \left(\{z\} \cup u_{1} \uparrow n_{1}\right) \cap \operatorname{keys}\left(m_{1}\right) \\
\equiv & u_{1} \uparrow n_{1} \cap \operatorname{keys}\left(m_{1}\right) \\
\equiv & \left(\operatorname{since} n_{2} \equiv n_{1} \ominus z \text { and } z \in \operatorname{keys}(n) \text { so that } z \notin u_{1}\right) \\
& u_{1} \uparrow n_{2} \cap \operatorname{keys}\left(m_{1}\right) \\
\equiv & u_{1} \uparrow n_{2} \cap \operatorname{keys}\left(n_{2}\right)
\end{aligned}
$$

and

$$
\begin{aligned}
& \uparrow \operatorname{block}(x, g) \cap \operatorname{keys}\left(m_{1}\right) \\
\equiv & \uparrow n \mid u_{2} \cap \operatorname{keys}\left(m_{1}\right) \\
\equiv & u_{2} \uparrow n \cap \operatorname{keys}\left(m_{1}\right) \\
\equiv & \left(\operatorname{since} n(y) \equiv z \notin \operatorname{keys}\left(m_{1}\right)\right) \\
& u_{2} \uparrow n_{1} \cap \operatorname{keys}\left(m_{1}\right) \\
\equiv & \left(\operatorname{since} z \notin u_{2}\right) \\
& u_{2} \uparrow n_{2} \cap \operatorname{keys}\left(m_{1}\right) \\
\equiv & u_{2} \uparrow n_{2} \cap \operatorname{keys}\left(n_{2}\right)
\end{aligned}
$$

and thus indeed $\left(u_{1} \cup u_{2}\right) \uparrow n_{2} \cap k e y s\left(n_{2}\right) \equiv t_{1}$. Furthermore, $m_{1} \equiv \operatorname{blockrep}\left(G_{1}, g\right) \equiv m \backslash$ block $(x, g)$ and $u_{2} \subseteq \downarrow \operatorname{block}(x, g)$ so that $\left(u_{1} \cup u_{2}\right) \cap \downarrow m_{1} \equiv \emptyset$. Therefore in this case

$$
\text { inreach2 }(G, g, n, m, u) \equiv[y \mapsto z] \cup \text { inreach2 }\left(G_{1}, g, n_{2}, m_{1}, u_{1} \cup u_{2}\right) \text {. }
$$

Altogether, we have obtained the following recurrent recursion for inreach2:

inreach2 $(G, g, n, m, u)$

$\supseteq P_{2}(G, g, n, m, u) \triangleright$

if $u=\emptyset$ 


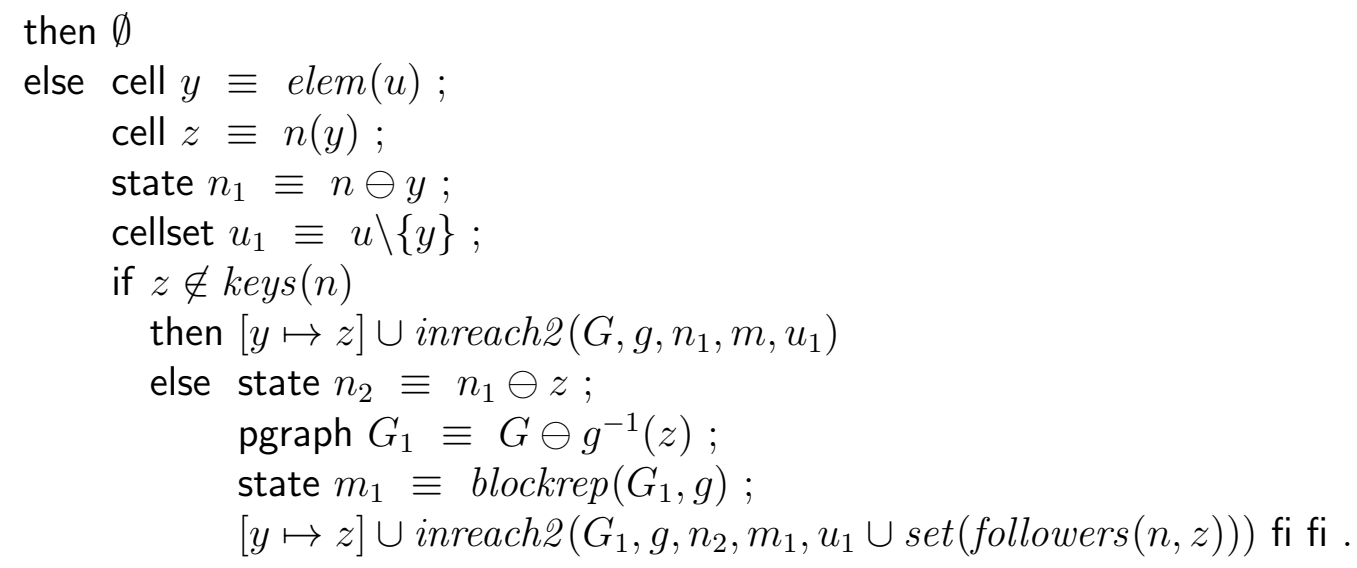

According to the rule (DESCENDANT-FIXPOINT) now the least fixpoint of the corresponding functional is a descendant of inreach2. We will denote this fixpoint again by inreach2 .

Obviously, the parameters $G, g$, and $m$ do not contribute to the computation proper and thus may be eliminated. We define recursively

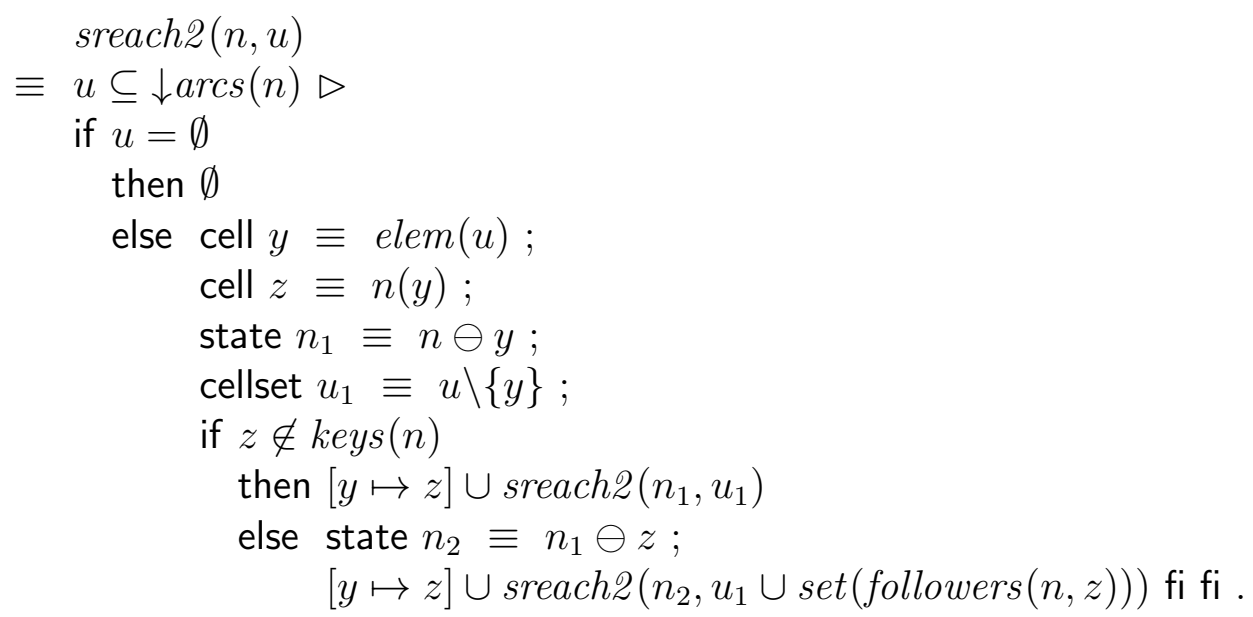

A trivial computational induction proves that

$$
\operatorname{sreach2}(n, u) \equiv \operatorname{inreach2}(G, g, n, m, u)
$$

provided $P_{2}(G, g, n, m, u)$ holds. Now we have

$$
\operatorname{arcs}\left(n_{1}\right)
$$

$\equiv$ inreach2 $(\tilde{G}, g, \tilde{n}, m, u)$

$\equiv \operatorname{sreach2}(\tilde{n}, u)$

$\equiv \operatorname{sreach} 2(n, u)$

Therefore we define the function

$$
\begin{aligned}
\operatorname{sreach}(n, t) \stackrel{\text { def }}{\equiv} & t \subseteq \operatorname{keys}(n) \triangleright \\
& \operatorname{sreach2}(n, \operatorname{setfollowers}(n, t)),
\end{aligned}
$$

which obviously satisfies (SR).

\subsection{Specialization to a Depth-First Traversal}

We now represent sets $u \subseteq \downarrow \operatorname{arcs}(n)$ of cells by sequences $\alpha$ such that $\operatorname{set}(\alpha) \equiv u$. Furthermore we concretize elem to first, replace set union by concatenation and obtain in this way a first deterministic algorithm for our problem. We specify sreach3 by

$$
\operatorname{sreach} 3(n, \alpha) \stackrel{\text { def }}{\equiv} P_{3}(n, \alpha) \triangleright \operatorname{sreach2}(n, \operatorname{set}(\alpha))
$$


with the invariant

$$
\begin{aligned}
P_{3}(n, \alpha) & \stackrel{\text { def }}{\equiv} \operatorname{set}(\alpha) \subseteq \downarrow \operatorname{arcs}(n) \\
& \wedge \operatorname{repetitionfree~}(\alpha) \\
& \wedge \operatorname{setfollowers}(n, \operatorname{keys}(n)) \cap \operatorname{set}(\alpha)=\emptyset
\end{aligned}
$$

where

$$
\text { repetitionfree }(\alpha) \stackrel{\text { def }}{\Leftrightarrow} \operatorname{isinjective}\left(\bigcup_{i=1}^{|\alpha|}[i \mapsto \alpha[i]]\right) \text {. }
$$

The last conjunct of $P_{3}$ corresponds to the last conjunct of $P_{2}$ and is needed in proving invariance of repetitionfree $(\alpha)$. We have the embedding

$$
\operatorname{sreach2}(n, u) \equiv \operatorname{sreach} 3(n, \operatorname{sort}(u)) .
$$

Using again the rule (DESCENDANT-FIXPOINT) we immediately obtain the recursion

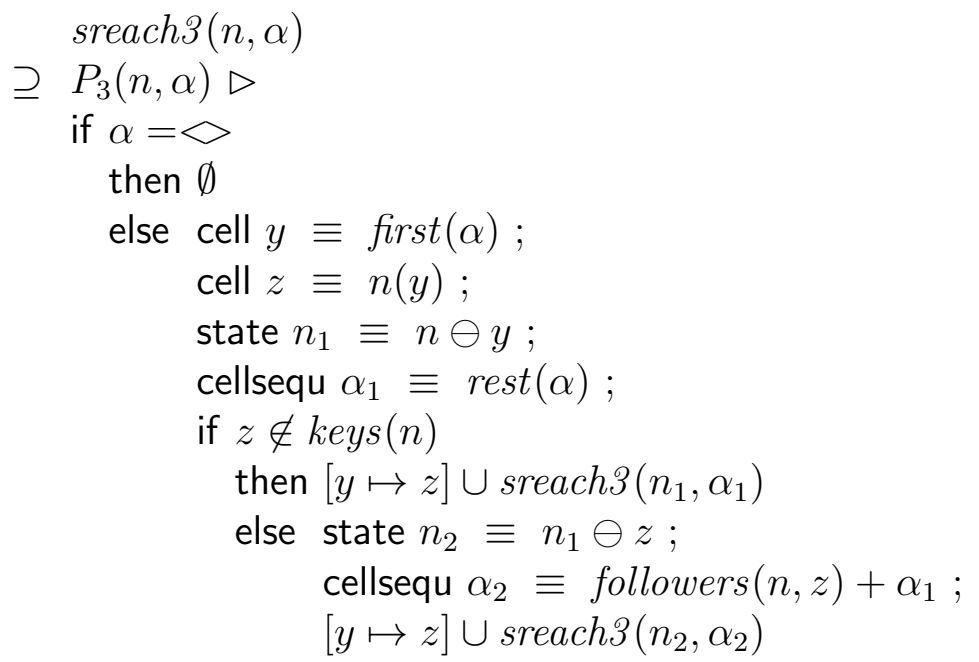

fi fi .

The choice $y \equiv \operatorname{first}(\alpha)$ (rather than $y \equiv$ last $(\alpha)$ ) leads to a depth-first traversal.

\subsection{Encoding the Arcs}

We have started this chapter considering a (fixed) pseudo-graph state $n_{0}$. From now on we will assume that the keys of $n_{0}$ are marked (for instance, using a bit-vector). We define, for $n \subseteq n_{0}$,

$$
\begin{array}{lll}
\operatorname{bound}_{(n)} & \stackrel{\text { def }}{\equiv} & \sup (\downarrow n)+1, \\
\operatorname{keys}_{+}(n) & \stackrel{\text { def }}{\equiv} & \operatorname{keys}(n) \cup\{\text { bound }(n)\}, \\
\operatorname{next}_{n}(z) & \stackrel{\text { def }}{\equiv} & \operatorname{succ}_{\text {keys }_{+}(n)}(z), \\
\text { keys }_{+} & \stackrel{\text { def }}{\equiv} & \operatorname{keys}_{+}\left(n_{0}\right), \\
\text { bound } & \stackrel{\text { def }}{\equiv} & \operatorname{bound}\left(n_{0}\right) .
\end{array}
$$

Obviously all submaps $n \subseteq n_{0}$ appearing in our reachability algorithms satisfy

$$
\operatorname{keys}(n) \subseteq \text { keys }_{+} \wedge \downarrow \operatorname{arcs}(n) \cap \text { keys }_{+} \equiv \emptyset .
$$

Moreover, we observe that the property

$$
\exists \beta: \alpha \equiv \text { followers }(n, \beta),
$$


where

$$
\text { followers }(m, \beta) \equiv \sum_{i \in[1:|\beta|]} \text { followers }(m, \beta[i])
$$

(see 3.2 for followers of single cells) is an invariant of sreach3. Therefore we can reconstruct the input $\alpha$ from the sequence $\beta$. Packing some other obvious invariants into the assertion

$$
\begin{aligned}
P_{4}(n, \beta) & \stackrel{\text { def }}{=} \operatorname{keys}(n) \subseteq \text { keys }_{+} \\
& \wedge \downarrow \operatorname{arcs}(n) \cap \text { keys }_{+}=\emptyset \\
& \wedge \operatorname{repetitionfree}(\beta) \\
& \wedge \downarrow n \cap \operatorname{set}(\beta)=\emptyset \\
& \wedge \forall z \in \operatorname{keys}(n) \cup \operatorname{set}(\beta):] z: \operatorname{next}_{n}(z)[\subseteq \downarrow \operatorname{arcs}(n) \backslash \operatorname{set}(\beta),
\end{aligned}
$$

we define

$$
\operatorname{sreach}_{4}(n, \beta) \stackrel{\text { def }}{\equiv} P_{4}(n, \beta) \triangleright \operatorname{sreach} 3(n, \operatorname{followers}(n, \beta)) \text {. }
$$

We obtain for $n \subseteq n_{0}$ and $t \subseteq$ keys $(n)$ the embedding

$$
\begin{aligned}
& \operatorname{sreach}(n, t) \\
\equiv & \operatorname{sreach} 2(n, \operatorname{setfollowers}(n, t)) \\
\equiv & \operatorname{sreach} 2(n, \operatorname{set}(\text { followers }(n, \operatorname{sort}(t)))) \\
\equiv & \operatorname{sreach} 3(n, \text { followers }(n, \operatorname{sort}(t))) \\
\equiv & \operatorname{sreach} 4(n, \operatorname{sort}(t)) .
\end{aligned}
$$

Now let $n$ and $\beta$ be such that $P_{4}(n, \beta)$ holds. Assume $\beta \not \equiv \diamond$ and let

$$
\begin{aligned}
& y \stackrel{\text { def }}{\equiv} \operatorname{first}(\beta)+1, \\
& \gamma \stackrel{\text { def }}{\equiv} \operatorname{rest}(\beta) .
\end{aligned}
$$

Case 1: $y \notin \downarrow \operatorname{arcs}(n)$.

Then followers $(n, \operatorname{first}(\beta)) \equiv \diamond$ and therefore

$$
\text { followers }(n, \beta) \equiv \operatorname{followers}(n, \gamma) \text {. }
$$

Hence

$$
\operatorname{sreach}_{4}(n, \beta) \equiv \operatorname{sreach}_{4}(n, \gamma) .
$$

Case 2: $y \in \downarrow \operatorname{arcs}(n)$.

Then, setting

$$
\alpha \stackrel{\text { def }}{\equiv} \text { followers }(n, \beta)
$$

we have

$$
y \equiv \operatorname{first}(\alpha)
$$

and

$$
\text { followers }(<y>+\gamma) \equiv \operatorname{rest}(\alpha)
$$

Therefore, if $z \in \operatorname{keys}(n)$ - where $z \stackrel{\text { def }}{\equiv} n(y)$ - then

$$
\text { followers }(n,<z>+<y>+\gamma) \equiv \operatorname{followers}(n, z)+\operatorname{rest}(\alpha) \text {. }
$$


The test $y \in \downarrow \operatorname{arcs}(n)$ may be simplified as follows: $P_{4}$ implies that

$$
\forall z \in \operatorname{set}(\beta):] z: \operatorname{next}_{n}(z)[\subseteq \downarrow \operatorname{arcs}(n),
$$

which, in turn, entails that

$$
y \notin \text { keys }_{+} \Rightarrow y \in \downarrow \operatorname{arcs}(n) .
$$

Hence

$$
y \notin \downarrow \operatorname{arcs}(n) \Leftrightarrow y \in \text { keys }_{+} .
$$

Therefore

$$
\begin{aligned}
& \operatorname{sreach}_{4}(n, \beta) \\
& \equiv P_{4}(n, \beta) \triangleright \\
& \text { if } \beta=\varnothing \\
& \text { then } \emptyset \\
& \text { else cell } y \equiv \operatorname{first}(\beta)+1 \text {; } \\
& \text { cellsequ } \gamma \equiv \operatorname{rest}(\beta) \text {; } \\
& \text { if } y \in \text { keys }_{+} \\
& \text {then } \operatorname{sreach} 4(n, \gamma) \\
& \text { else cell } z \equiv n(y) \text {; } \\
& \text { state } n_{1} \equiv n \ominus y \text {; } \\
& \text { cellsequ } \beta_{1} \equiv<y>+\gamma \text {; } \\
& \text { if } z \notin \operatorname{keys}(n) \\
& \text { then }[y \mapsto z] \cup \operatorname{sreach} 4\left(n_{1}, \beta_{1}\right) \\
& \text { else state } n_{2} \equiv n_{1} \ominus z \text {; } \\
& \text { cellsequ } \beta_{2} \equiv\langle z\rangle+\beta_{1} \text {; } \\
& {[y \mapsto z] \cup \operatorname{sreach} 4\left(n_{2}, \beta_{2}\right) \text { fi fi fi . }}
\end{aligned}
$$

\subsection{Integration of Chaining}

Next we transform sreach 4 into a tail recursion and add chaining. In a first step we add a parameter $m$ that accumulates - not, as usual, the intermediate results themselves, but - chainings of the intermediate results. This will make the chaining step very easy. We specify

$$
\operatorname{sreach} 5(n, \beta, m) \stackrel{\text { def }}{\equiv} P_{5}(n, \beta, m) \triangleright \operatorname{unchain}(m) \cup \operatorname{sreach}_{4}(n, \beta) \text {. }
$$

The assertion $P_{5}$ collects all the invariants we need for the further development; in it we use the function

$$
\operatorname{cinner}(m) \stackrel{\text { def }}{=} \bigcup_{x \in \operatorname{src}(m)} \operatorname{inner}(\operatorname{from}(x, m)) \text {. }
$$

Then

$$
\begin{aligned}
P_{5}(n, \beta, m) & \stackrel{\text { def }}{=} P_{4}(n, \beta) \\
& \wedge \text { ischaining }(m) \\
& \wedge \operatorname{cinner}(m) \cap \operatorname{keys}(n)=\emptyset \\
& \wedge \downarrow n \cap \downarrow m=\emptyset \\
& \wedge \operatorname{arcs}(n) \subseteq \operatorname{keys}(n) \cup \operatorname{src}(m) \\
& \wedge \beta \neq \emptyset \operatorname{keys}_{+}(n) \cup \operatorname{src}(m) \\
& \wedge \operatorname{src}(m)=\{z \in \operatorname{set}(\operatorname{rest}(\beta)) \subseteq \square \downarrow m \\
& \wedge \operatorname{cinner}(m)= \\
& \bigcup \bigcup\{] z: y] \mid z \in \operatorname{set}(\beta) \cap\left[z: \operatorname{next}_{n}(z)[\neq \emptyset\} \wedge y \in\right] z: \operatorname{next}_{n}(z)[\cap \operatorname{set}(\beta)\}
\end{aligned}
$$


We have the embedding

$$
\operatorname{sreach}\left(n_{0}, t\right) \equiv \operatorname{sreach} 5\left(n_{0}, \operatorname{sort}(t),[t \mapsto \square]\right) .
$$

Note that unchain $([t \mapsto \square]) \equiv \emptyset$. Recall now from Section 6.3.3 that for a chaining $m$ and for $y, z \notin \operatorname{cinner}(m)$

$$
\operatorname{unchain}(m) \cup[y \mapsto z] \equiv \operatorname{unchain}(\operatorname{insert}(m, z, y))
$$

where

$$
\begin{array}{r}
\operatorname{insert}(m, z, y) \equiv \text { if } z \in \operatorname{src}(m) \text { then } m \nLeftarrow([z \mapsto y] \cup[y \mapsto m(z)]) \\
\text { else } m \nLeftarrow([z \mapsto y] \cup[y \mapsto \square]) \quad \text { fi . }
\end{array}
$$

Therefore we obtain the recursion

$$
\begin{aligned}
& \text { sreach } 5(n, \beta, m) \\
& \equiv P_{5}(n, \beta, m) \triangleright \\
& \text { if } \beta=\varnothing \\
& \text { then } \operatorname{unchain}(m) \\
& \text { else cell } y \equiv \operatorname{first}(\beta)+1 \text {; } \\
& \text { cellsequ } \gamma \equiv \operatorname{rest}(\beta) \text {; } \\
& \text { if } y \in k e y s_{+} \\
& \text {then } \operatorname{sreach} 5(n, \gamma, m) \\
& \text { else cell } z \equiv n(y) \text {; } \\
& \text { state } n_{1} \equiv n \ominus y \text {; } \\
& \text { cellsequ } \beta_{1} \equiv<y>+\gamma \text {; } \\
& \text { state } m_{1} \equiv \operatorname{insert}(m, z, y) \text {; } \\
& \text { if } z \notin \operatorname{keys}(n) \\
& \text { then sreach } 5\left(n_{1}, \beta_{1}, m_{1}\right) \\
& \text { else state } n_{2} \equiv n_{1} \ominus z \text {; } \\
& \text { cellsequ } \beta_{2} \equiv<z>+\beta_{1} \text {; } \\
& \text { sreach } 5\left(n_{2}, \beta_{2}, m_{1}\right) \text { fi fi fi . }
\end{aligned}
$$

Now chaining can be added simply by replacing unchain $(m)$ by $m$ in the body of sreach 5 . We take $P_{6} \equiv P_{5}$ and define

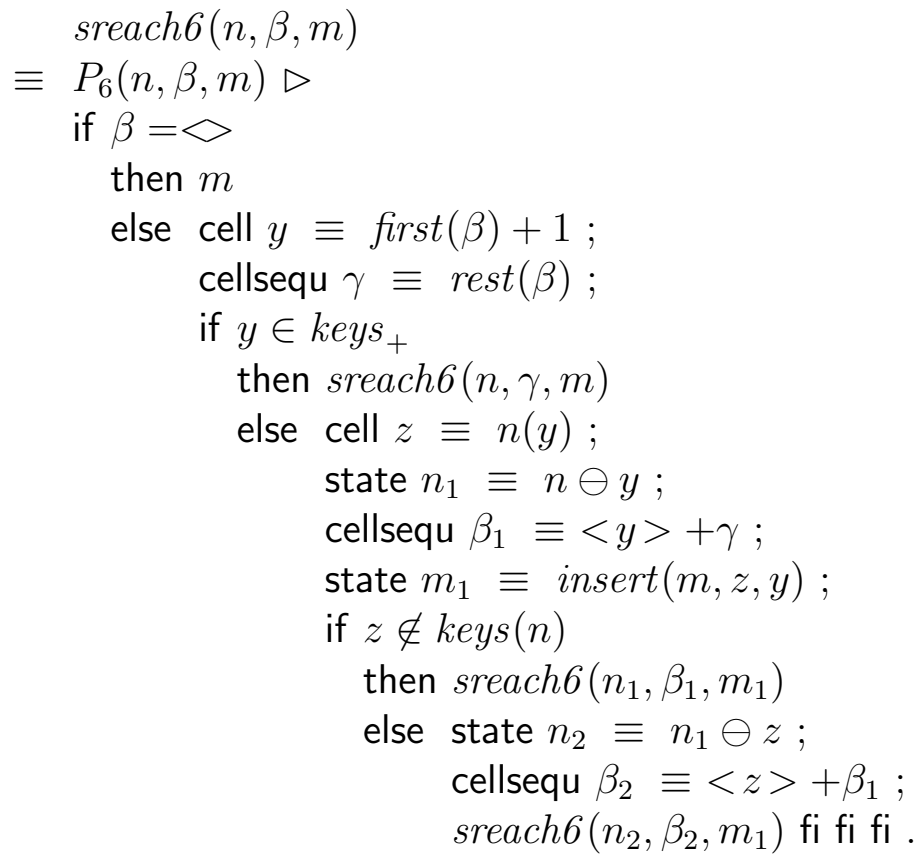


Now we have

$$
\operatorname{sreach} 5(n, \beta, m) \equiv \operatorname{unchain}(\operatorname{sreach} 6(n, \beta, m)),
$$

(a proof of this fact is provided by the implementation rule RANGE in [Berghammer, Ehler 90]) and consequently

$$
\operatorname{sreach}\left(n_{0}, t\right) \equiv \operatorname{unchain}\left(\operatorname{sreach} 6\left(n_{0}, \operatorname{sort}(t),[t \mapsto \square]\right)\right)
$$

Therefore

$$
\operatorname{csreach}(n, t) \stackrel{\text { def }}{\equiv} \operatorname{sreach} 6(n, \operatorname{sort}(t),[t \mapsto \square])
$$

satisfies the required equation (CSR) from Section 7.1.

\subsection{Improving Space Efficiency}

As standing, the algorithm for csreach still uses the three "large" parameters $n, \beta$ and $m$. In the case of garbage collection, however, there is no space available for these parameters. So we need to represent them by one map parameter, viz. by the store itself. The assertion $P_{5}\left(\equiv P_{6}\right)$ clearly indicates how to proceed: The conjunct $\downarrow m \cap \downarrow n=\emptyset$ allows us to simply unify $n$ and $m$; moreover, since $\operatorname{set}(\operatorname{rest}(\beta)) \subseteq \square \downarrow m$ we may overwrite $m$ with (a map representation of) rest( $\beta$ ) without losing essential information about $m$. To this end, we define (using operations from Section 6.3) for sequences $\gamma \in(\text { cell } \backslash\{\square\})^{*}$

$$
\begin{aligned}
\operatorname{map}(\gamma) \stackrel{\text { def }}{\equiv} & \text { repetitionfree }(\gamma) \triangleright \\
& \text { if } \gamma=\diamond \text { then } \emptyset \text { else } \operatorname{prefix}(\operatorname{first}(\gamma), \operatorname{map}(\operatorname{rest}(\gamma))) \mathrm{fi}
\end{aligned}
$$

and for maps $c$ and cells $v$ such that isanchored $(c) \wedge v \in \operatorname{set}(c)$,

$$
\operatorname{seq}(v, c) \stackrel{\text { def }}{\equiv} \text { if } v=\square \text { then } \diamond \text { else }\langle v>+\operatorname{seq}(c(v), c \ominus v) \mathrm{fi} \text {. }
$$

If $v \equiv \operatorname{first}(c)$ this may be rewritten as

$$
\operatorname{seq}(v, c) \equiv \text { if } v=\square \text { then } \diamond \text { else }\langle v>+\operatorname{seq}(c(v), \operatorname{rest}(c)) \mathrm{fi} .
$$

Then obviously

$$
\operatorname{seq}(\operatorname{tirst}(\gamma), \operatorname{map}(\gamma)) \equiv \gamma
$$

where

$$
\operatorname{tfirst}(\gamma) \stackrel{\text { def }}{\equiv} \text { if } \gamma=\diamond \text { then } \square \text { else } \operatorname{first}(\gamma) \mathrm{fi}
$$

and

$$
\operatorname{set}(\gamma) \stackrel{\text { def }}{\equiv} \downarrow \operatorname{map}(\gamma) .
$$

Hence we have isanchored $(\operatorname{map}(\gamma))$ and $\operatorname{map}(\gamma)$ represents $\operatorname{set}(\gamma)$.

We want to represent the sequence $\beta$ in sreach 6 by the triple $(y, v, c)$ where

$$
\begin{aligned}
& y \stackrel{\text { def }}{\equiv} \operatorname{tfirst}(\beta)+1 \text {, } \\
& v \stackrel{\text { def }}{\equiv} \operatorname{tfirst}(\operatorname{trest}(\beta)) \text {, } \\
& c \stackrel{\text { def }}{\equiv} \operatorname{map}(\operatorname{trest}(\beta))
\end{aligned}
$$

and

$$
\operatorname{trest}(\gamma) \stackrel{\text { def }}{\equiv} \text { if } \gamma=\diamond \text { then } \diamond \operatorname{else} \operatorname{rest}(\gamma) \text { fi } .
$$


We can retrieve $\beta$ from $(y, v, c)$ by virtue of

$$
\beta \equiv<y-1>+\operatorname{seq}(v, c) .
$$

Hence we define

$$
\operatorname{sreach} 7(n, y, v, c, m) \stackrel{\text { def }}{\equiv} P_{7}(n, y, v, c, m) \triangleright \operatorname{sreach} 6(n,<y-1>+\operatorname{seq}(v, c), m)
$$

where

$$
\begin{aligned}
P_{7}(n, y, v, c, m) & \stackrel{\text { def }}{\equiv} \text { isanchored }(c) \wedge v \in \operatorname{set}(c) \\
& \wedge P_{5}(n,<y-1>+\operatorname{seq}(v, c), m) \\
& \wedge y \notin \downarrow c \\
& \wedge \operatorname{set}(\operatorname{seq}(v, c))=\downarrow c .
\end{aligned}
$$

We have

$$
\operatorname{sreach}\left(n_{0}, t\right) \equiv \operatorname{unchain}\left(\operatorname{sreach} 7\left(n_{0}, y, v, c, m\right)\right)
$$

where

$$
\begin{aligned}
& y \stackrel{\text { def }}{\equiv} \operatorname{tmin}(t)+1 \text {, } \\
& v \stackrel{\text { def }}{\equiv} \operatorname{tmin}(t \backslash\{\operatorname{tmin}(t)\}) \text {, } \\
& c \stackrel{\text { def }}{\equiv} \operatorname{map}(t \backslash\{\operatorname{tmin}(t)\}) \text {, } \\
& m \stackrel{\text { def }}{\equiv}[t \mapsto \square]
\end{aligned}
$$

and

$$
\operatorname{tmin}(t) \stackrel{\text { def }}{=} \min (t \cup\{\square\}) .
$$

Note that $v \equiv \operatorname{first}(c)$. To find a recursion for sreach'7 we consider values $n, y, v, c, m$ satisfying the invariant $P_{7}(n, y, v, c, m)$. Define

$$
\beta \equiv<y-1>+\operatorname{seq}(v, c)
$$

Then

$$
\begin{aligned}
& y \equiv \operatorname{first}(\beta)+1 \\
& \operatorname{seq}(v, c) \equiv \operatorname{rest}(\beta) .
\end{aligned}
$$

Case 1: $y \in k e y s_{+}$.

Then

$$
\operatorname{sreach} 7(n, y, v, c, m) \equiv \operatorname{sreach} 6(n, \operatorname{seq}(v, c), m) .
$$

If $v \equiv \square$ then $\operatorname{seq}(v, c) \equiv \diamond$ and hence

$$
\operatorname{sreach} 6(n, \operatorname{seq}(v, c), m) \equiv m \text {. }
$$

Otherwise,

$$
\operatorname{seq}(v, c) \equiv<v>+\operatorname{seq}(c(v), \operatorname{rest}(c)),
$$

which implies that

$$
\operatorname{sreach} 6(n, \operatorname{seq}(v, c), m) \equiv \operatorname{sreach} 7(n, v+1, c(v), \operatorname{rest}(c), m) .
$$

Case 2: $y \notin k e y s_{+}$.

Define

$$
z \stackrel{\text { def }}{\equiv} n(y) \text { and } m_{1} \stackrel{\text { def }}{\equiv} \operatorname{insert}(m, z, y) \text {. }
$$

If $z \notin \operatorname{keys}(n)$ 


$$
\begin{aligned}
& \operatorname{sreach} 7(n, y, v, c, m) \\
\equiv & \operatorname{sreach} 6\left(n \ominus y,<y>+\operatorname{rest}(\beta), m_{1}\right) \\
\equiv & \operatorname{sreach} 6\left(n \ominus y,<y>+\operatorname{seq}(v, c), m_{1}\right) \\
\equiv & \operatorname{sreach} 7\left(n \ominus y, y+1, v, c, m_{1}\right) .
\end{aligned}
$$

Otherwise,

$$
\begin{aligned}
& \operatorname{sreach} 7(n, y, v, c, m) \\
\equiv & \operatorname{sreach} 6\left(n \ominus y \ominus z,<z>+<y>+\operatorname{seq}(v, c), m_{1}\right) \\
\equiv & \operatorname{sreach} 7\left(n \ominus y \ominus z, z+1, y, \operatorname{prefix}(y, c), m_{1}\right)
\end{aligned}
$$

because $y \notin \downarrow c$ implies

$$
\operatorname{seq}(y, \operatorname{prefix}(y, c)) \equiv<y>+\operatorname{seq}(v, \operatorname{rest}(\operatorname{prefix}(y, c))) \equiv<y>+\operatorname{seq}(v, c) .
$$

Hence we obtain the recursion

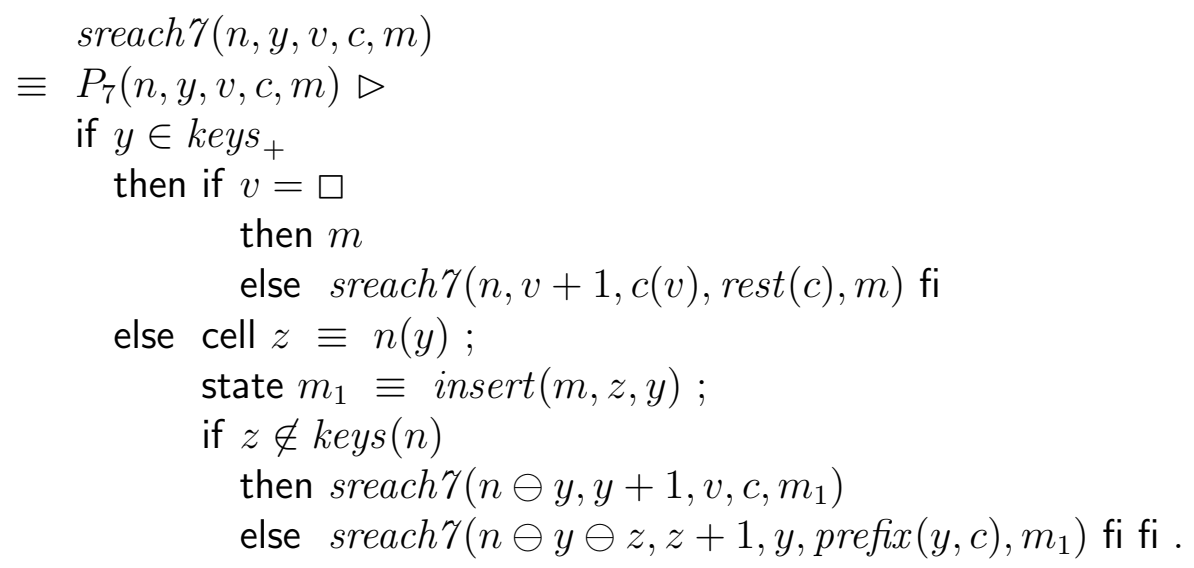

Now we unite the parameters $n, c, m$ into one map parameter $l$. We define

$$
\operatorname{sreach} 8(n, y, v, c, m, l) \stackrel{\text { def }}{\equiv} P_{8}(n, y, v, c, m, l) \triangleright n \varangle \operatorname{sreach} 7(n, y, v, c, m)
$$

where

$$
P_{8}(n, y, v, c, m, l) \stackrel{\text { def }}{=} P_{7}(n, y, v, c, m) \wedge l=n \cup(m \varangle c) .
$$

Let now $n, y, v, c, m, l$ be such that $P_{8}(n, y, v, c, m, l)$ holds.

Case 1: $y \in$ keys $_{+}$.

If $v \equiv \square$ then $c \equiv \emptyset$ and hence

$$
\operatorname{sreach} 8(n, y, v, c, m, l) \equiv n \nleftarrow m \equiv n \cup m \equiv l .
$$

Otherwise,

$$
\operatorname{sreach} 8(n, y, v, c, m, l) \equiv \operatorname{sreach} 8(n, v+1, l(v), \operatorname{rest}(c), m, l \nLeftarrow[v \mapsto \square])
$$

because $c(v) \equiv l(v)$ and $v \in \downarrow c \subseteq \square \downarrow m$ together with $n \cup(m \varangle c) \equiv l$ implies $n \cup(m \varangle \operatorname{rest}(c))$ $\equiv l \triangleleft[v \mapsto \square]$.

Case 2: $y \notin$ keys $_{+}$.

Define $z \stackrel{\text { def }}{\equiv} n(y) \equiv l(y)$ and $m_{1} \stackrel{\text { def }}{\equiv} \operatorname{insert}(m, z, y)$. If $z \notin k e y s(n)$ then

$$
\operatorname{sreach} 8(n, y, v, c, m, l) \equiv \operatorname{sreach} 8\left(n \ominus y, y+1, v, c, m_{1}, n \ominus y \cup\left(m_{1} \varangle c\right)\right) .
$$

Since $z \in \uparrow \operatorname{arcs}(n) \subseteq \operatorname{keys}(n) \cup \operatorname{src}(m)$ and $\operatorname{cinner}(m) \cap k e y s_{+} \equiv \emptyset$, we have

$$
z \notin \operatorname{keys}(n) \Leftrightarrow z \in \operatorname{src}(m) \Leftrightarrow m(z) \not \equiv \square \Leftrightarrow l(z) \not \equiv \square .
$$


Moreover, $z \in \operatorname{src}(m)$ implies

$$
m_{1} \equiv m \nLeftarrow([z \mapsto y] \cup[y \mapsto m(z)])
$$

by definition of insert. Furthermore, since

$$
\downarrow c \equiv \operatorname{set}(\operatorname{seq}(v, c)) \subseteq \square \downarrow m
$$

and $y, z \notin \square \downarrow m$, we get $y, z \notin \downarrow c$ and therefore

$$
m_{1} \nleftarrow c \equiv(m \varangle c) \triangleleft([z \mapsto y] \cup[y \mapsto m(z)]) .
$$

Hence

$$
n \ominus y \cup\left(m_{1} \nleftarrow c\right) \equiv l \nLeftarrow([z \mapsto y] \cup[y \mapsto m(z)]) .
$$

If $z \in \operatorname{keys}(n)$ (i.e., if $l(z) \equiv \square)$ then

$$
\operatorname{sreach} 8(n, y, v, c, m, l) \equiv \operatorname{sreach} 8\left(n \ominus y \ominus z, z+1, y, \operatorname{prefix}(y, c), m_{1}, l_{1}\right)
$$

where

$$
l_{1} \stackrel{\text { def }}{\equiv} n \ominus y \ominus z \cup\left(m_{1} \nLeftarrow \operatorname{prefix}(y, c)\right) \text {. }
$$

Since $z \notin \operatorname{src}(m)$,

$$
m_{1} \equiv m \varangle([z \mapsto y] \cup[y \mapsto \square]) .
$$

Furthermore, since $z \in k e y s_{+}$and inner $(m) \cap k e y s_{+} \equiv \emptyset$, we get $z \notin \downarrow m$ and thus again $z \notin \downarrow c$. Therefore

$$
l_{1} \equiv l \nLeftarrow([z \mapsto y] \cup[y \mapsto v])
$$

Summarizing this we obtain

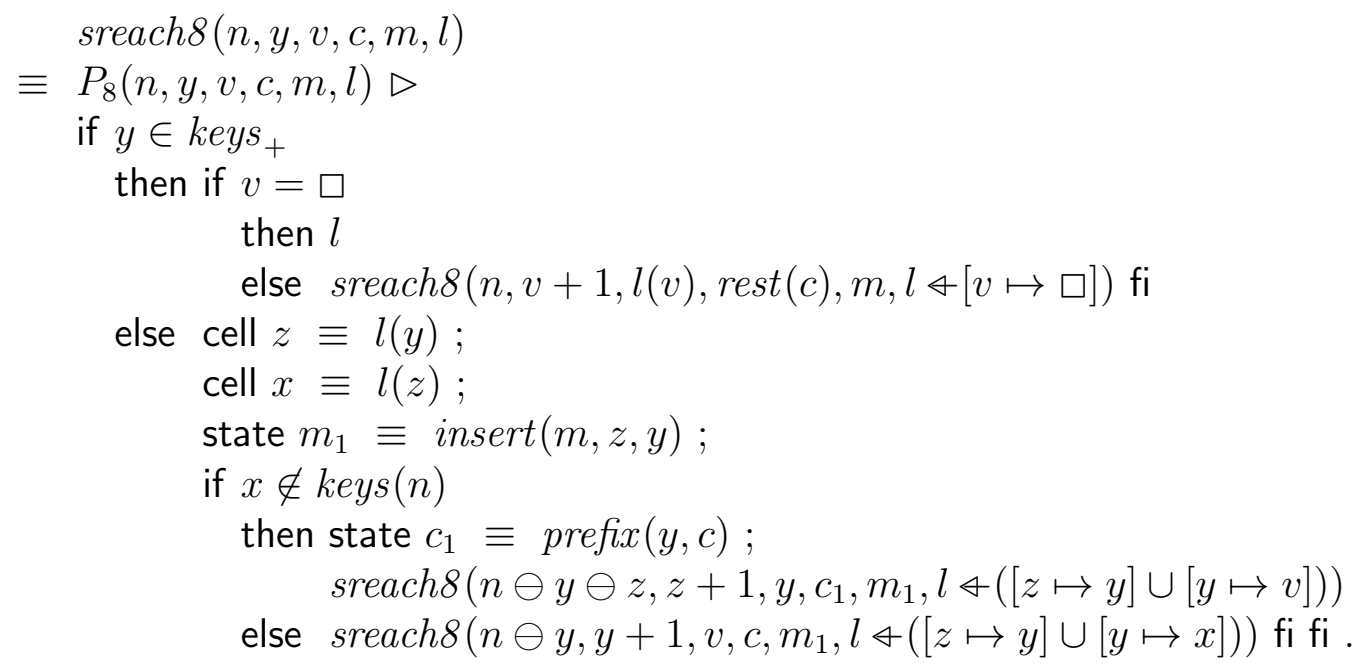

Eliminating the redundant parameters $n, c, m$ we obtain our final algorithm

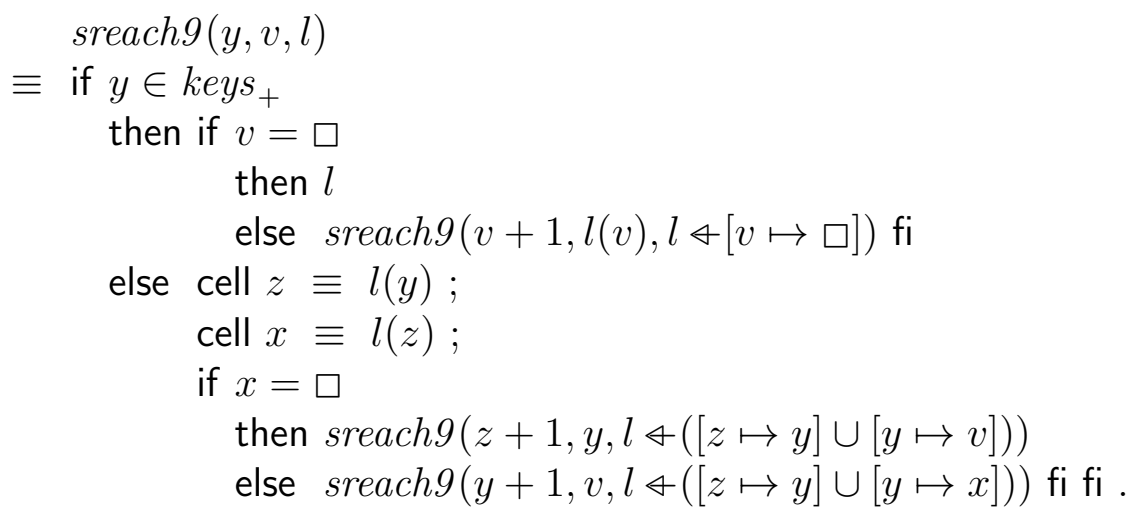




\subsection{Assembling the Parts}

We have to follow the chain of embeddings to obtain the final program for sreach. For $n, y, v, c, m$ such that $P_{7}(n, y, v, c, m)$ holds we have

$$
\begin{aligned}
& n \nLeftarrow \operatorname{sreach} 7(n, y, v, c, m) \\
\equiv & \operatorname{sreach} 8(n, y, v, c, m, n \cup(m \varangle c)) \\
\equiv & \operatorname{sreach}(y, v, n \cup(m \varangle c)) .
\end{aligned}
$$

In the sequel we will - for convenience - consider only singleton sets $s \equiv\left\{z_{0}\right\}$ (denoted just by $z_{0}$ ) of starting keys $z_{0} \in \operatorname{keys}\left(n_{0}\right)$. To see what sreach 9 really does, we define

$$
\begin{aligned}
& m_{0} \stackrel{\text { def }}{\equiv} \operatorname{sreach} 7\left(n_{0}, z_{0}+1, \square, \emptyset,\left[z_{0} \mapsto \square\right]\right), \\
& l_{0} \stackrel{\text { def }}{\equiv} \operatorname{sreach} 9\left(z_{0}+1, \square, n_{0}\right) .
\end{aligned}
$$

Since $n_{0} \cup\left(\left[z_{0} \mapsto \square\right] \cup \emptyset\right) \equiv n_{0} \cup\left[z_{0} \mapsto \square\right] \equiv n_{0}$ by $z_{0} \in$ keys $\left(n_{0}\right)$, we have $l_{0} \equiv n_{0} \nleftarrow m_{0}$ and unchain $\left(m_{0}\right) \equiv \operatorname{sreach}\left(n_{0}, z_{0}\right)$. Actually we want to compute $m_{0}$; but sreach 9 computes $l_{0}$ which is $m_{0}$ plus the garbage $l_{0} \ominus \downarrow m_{0}$. Thus we must try to retrieve $m_{0}$ from $l_{0}$. Let now

$$
\text { keys } \stackrel{\text { def }}{\equiv} \operatorname{keys}\left(n_{0}\right) \text {. }
$$

The invariant $P_{8}$ implies

$$
\begin{aligned}
& \text { keys } \subseteq \downarrow l_{0}, \\
& \operatorname{src}\left(m_{0}\right) \equiv\left\{z \in \text { keys } \mid l_{0}(z) \not \equiv \square\right\}
\end{aligned}
$$

Furthermore,

$$
\downarrow m_{0} \equiv \bigcup_{z \in \operatorname{src}\left(m_{0}\right)} \operatorname{ginner}\left(l_{0}, z\right)
$$

because $m_{0}$ is a chaining (cf. Section 6.3.4). Hence

$$
\begin{aligned}
& m_{0} \\
\equiv & l_{0} \mid \downarrow m_{0} \\
\equiv & l_{0} \mid\left(\bigcup\left\{\text { ginner }\left(l_{0}, z\right) \mid z \in \text { keys } \wedge l_{0}(z) \not \equiv \square\right\}\right) .
\end{aligned}
$$

We set

$$
\text { retrieve }\left(l_{0}\right) \stackrel{\text { def }}{\equiv} l_{0} \mid\left(\bigcup\left\{\text { ginner }\left(l_{0}, z\right) \mid z \in \text { keys } \wedge l_{0}(z) \not \equiv \square\right\}\right) .
$$

However, the operation retrieve will not be executed; we will just use it for further development.

We define a function ocsreach (overwritten, chained state reachability) by

$$
\operatorname{ocsreach}(n, z) \stackrel{\text { def }}{\equiv} \operatorname{iscgstate}(n) \wedge z \in \operatorname{keys}(n) \triangleright \operatorname{sreach} 9(z+1, \square, n)
$$

(see Section 3.2 for iscgstate). We will not compute the test

$$
i s k e y_{+}(z) \stackrel{\text { def }}{\equiv} z \in k e y s_{+}
$$

occurring within sreach9, but regard it as an additional global parameter (to be realized e.g. by a bit vector). Thus the complete program reads

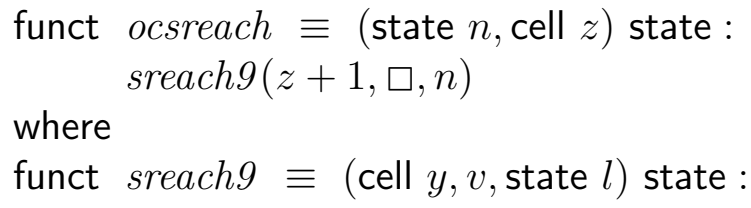




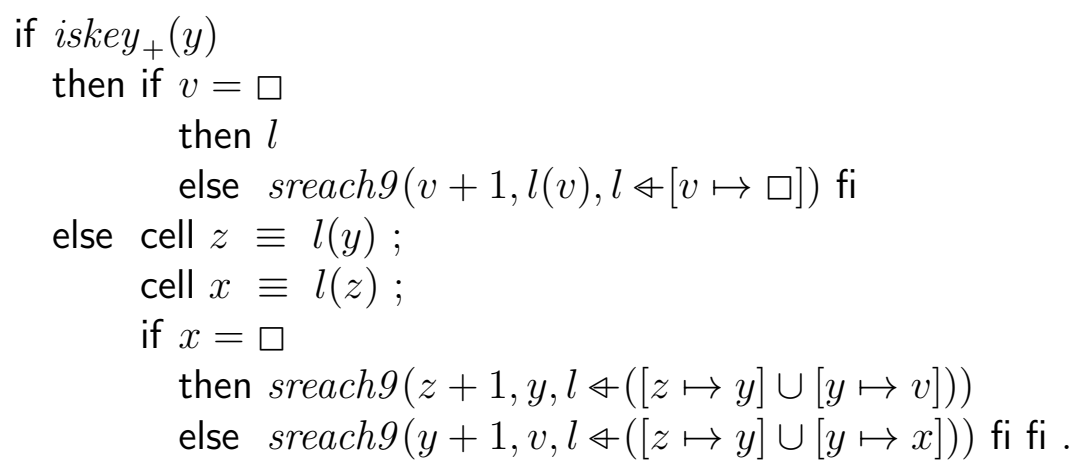

ocsreach meets the specification

$$
\operatorname{sreach}(n, z) \equiv \operatorname{unchain}(\text { retrieve }(\operatorname{ocsreach}(n, z)))
$$

if $n$ is a compressed state and $z \in \operatorname{keys}(n)$.

\section{Copying Chained Graph States}

In the preceding chapter we have shown how to compute from a compressed graph state

$$
n_{0} \equiv \operatorname{blockrep}\left(G_{0}, g\right) \text {, }
$$

a membership test iskey $y_{+}$for keys ${ }_{+}\left(n_{0}\right)$, and

$$
s \equiv\left\{z_{0}\right\} \subseteq \operatorname{keys}(n)
$$

a map

$$
l_{0} \equiv \operatorname{ocsreach}\left(n_{0}, \text { iskey }_{+}, z_{0}\right)
$$

such that

$$
m_{0} \stackrel{\text { def }}{\equiv} \text { retrieve }\left(l_{0}\right)
$$

is a chained representation of $\operatorname{arcs}\left(n_{1}\right)$ (so that $\operatorname{unchain}\left(m_{0}\right) \equiv \operatorname{arcs}\left(n_{1}\right) \equiv \operatorname{sreach}\left(n_{0}, s\right)$ ), where

$$
n_{1} \stackrel{\text { def }}{\equiv} \text { blockrep }\left(G_{s}, g\right)
$$

and

$$
G_{s} \stackrel{\text { def }}{\equiv} \operatorname{reach}\left(G_{0}, s\right)
$$

According to our problem analysis in Section 4.3 we are left with the task of computing the compressed state $n_{s} \equiv \operatorname{blockrep}\left(G_{s}, g_{s}\right)$ (where $g_{s}$ is a perfect allocation of $G_{s}$ ) from $n_{1}$ and the collapsing map $k$ defined by

$$
k(g(x)+i) \stackrel{\text { def }}{=} g_{s}(x)+i \quad\left(x \in \downarrow G_{s}, i \in[0:|G(x)|]\right) .
$$

We generalize this to the following problem: Given a graph $G$ and an overlap-free and orderpreserving (but not necessarily gap-free) allocation $g$, compute from

$$
n \stackrel{\text { def }}{\equiv} \operatorname{blockrep}(G, g)
$$

the compressed state

$$
n_{c} \stackrel{\text { def }}{\equiv} \operatorname{blockrep}\left(G, g_{c}\right)
$$


for the unique compressing allocation $g_{c}$ of $G$.

In Section 4.3 we have already seen that $n_{c}$ is the copy of $n$ via the collapsing map $k$; i.e., $n_{c} \equiv k \circ n \circ k^{-1}$, where $k$ now is defined by

$$
k(g(x)+i) \stackrel{\text { def }}{\equiv} g_{c}(x)+i
$$

for $x \in \downarrow G$ and $i \in[0:|G(x)|])$. Assuming, in accordance with the situation above, that we have a map $l$ such that $m \stackrel{\text { def }}{\equiv}$ retrieve $(l)$ is a chained representation of $\operatorname{arcs}(n)$, we have the decomposition

$$
\begin{aligned}
& n \\
\equiv & n_{\square} \cup \operatorname{arcs}(n) \\
\equiv & n_{\square} \cup \operatorname{unchain}(m)
\end{aligned}
$$

where

$$
n_{\square} \stackrel{\text { def }}{\equiv} n \mid \operatorname{keys}(n)(\equiv n \backslash \operatorname{arcs}(n)) \text {. }
$$

Now we can employ the functions derived in Chapter 6 to calculate

$$
\begin{aligned}
& n_{c} \\
\equiv & k \circ n \circ k^{-1} \\
\equiv & k \circ\left(n_{\square} \cup \operatorname{unchain}(m)\right) \circ k^{-1} \\
\equiv & \left(k \circ n_{\square} \circ k^{-1}\right) \cup\left(k \circ \text { unchain }(m) \circ k^{-1}\right) \\
\equiv & (\operatorname{since} k(\square) \equiv \square) \\
& \left(n_{\square} \circ k^{-1}\right) \cup \operatorname{copy}(m, k) \\
\equiv & \operatorname{copass}\left(n_{\square}, k\right) \cup \operatorname{copass}(\text { relocate }(m, k), k) .
\end{aligned}
$$

By the definition of copass we have

$$
\begin{aligned}
\equiv & \bigcup_{z \in \downarrow n_{\square}}^{\operatorname{copass}\left(n_{\square}, k\right)}\left[k(z) \mapsto n_{\square}(z)\right] \\
\equiv & \bigcup_{z \in \downarrow n_{\square}}[k(z) \mapsto \square] .
\end{aligned}
$$

Furthermore we define

$$
\begin{aligned}
& p \stackrel{\text { def }}{\equiv} \text { relocate }(m, k) \equiv k \circ \operatorname{arcs}(n), \\
& \operatorname{size}(z) \stackrel{\text { def }}{\equiv} \operatorname{succ}_{\text {keys }_{+}}(z)-z .
\end{aligned}
$$

Using that

$$
\begin{aligned}
& \downarrow p \\
\equiv & \downarrow \operatorname{arcs}(n) \\
\equiv & \bigcup_{z \in \operatorname{keys}(n)}[z+1: z+\operatorname{size}(z)]
\end{aligned}
$$

we obtain

$$
\begin{aligned}
\equiv & \bigcup_{x \in \downarrow p}^{\operatorname{copass}(p, k)}[k(x) \mapsto p(x)] \\
\equiv & \bigcup_{z \in \operatorname{keys}(n)} \bigcup_{i \in[1: \text { size }(z)]}[k(z+i) \mapsto p(z+i)] .
\end{aligned}
$$

Therefore 


$$
\begin{aligned}
& n_{c} \\
\equiv & \operatorname{copass}\left(n_{\square}, k\right) \cup \operatorname{copass}(p, k) \\
\equiv & \bigcup_{z \in \text { keys }(n)}\left([k(z) \mapsto \square] \cup\left(\bigcup_{i \in[1: \operatorname{size}(z)]}[k(z)+i \mapsto p(z+i)]\right)\right.
\end{aligned}
$$

since $k(z+i) \equiv k(z)+i$ for $i \in[1: \operatorname{size}(z)]$.

Thus our problem divides into two parts:

1. Compute $p \equiv$ relocate $(m, k)$ from $l$.

2. Compute the union above.

\subsection{Relocation Pass}

By the definition of relocate (cf. Section 6.3.4) it is immediate that

$$
\operatorname{relocate}(m, k) \equiv \bigcup_{z \in \text { keys }(n)} \operatorname{fibre}(m, z, k(z))
$$

where

$$
\text { fibre }(m, z, y) \equiv[\operatorname{ginner}(m, z) \mapsto y],
$$

because $\operatorname{src}(m) \equiv \operatorname{keys}(n)$. We aim at an algorithm that traverses the set keys $(n)$ in ascending order. Therefore we try to compute $k\left(\operatorname{next}_{n}(z)\right.$ ) (cf. Section 7.5) incrementally from $k(z)$ (for $z \in \operatorname{keys}(n) \cup\{\square\}$ such that $\stackrel{\vee}{z} \cap$ keys $_{+}(n) \backslash\{z\} \not \equiv \emptyset$; cf. Section 2.2 .2 for $\left.\stackrel{v}{z}\right)$. Let

$$
\begin{aligned}
& z_{1} \stackrel{\text { def }}{\equiv} \operatorname{next}_{n}(z), \\
& x \stackrel{\text { def }}{\equiv} g^{-1}(z) .
\end{aligned}
$$

Then, since $g$ is order-preserving,

$$
z_{1} \equiv \operatorname{next}_{n}(g(x)) \equiv g\left(\operatorname{succ}_{\downarrow_{G}}(x)\right) .
$$

Therefore

$$
\begin{aligned}
& k\left(z_{1}\right) \\
\equiv & k\left(g\left(\text { succ }_{\downarrow G}(x)\right)\right) \\
\equiv & (\text { by }(\mathrm{K})) \\
& g_{c}\left(\operatorname{succ}_{\downarrow G}(x)\right) \\
\equiv & (\text { by Theorem } 3.2 .6) \\
& g_{c}(x)+\operatorname{size}(x) \\
\equiv & (\text { since } n \equiv \text { blockrep }(G, g)) \\
& k(g(x))+\operatorname{size}(g(x)) \\
\equiv & k(z)+\operatorname{size}(z) .
\end{aligned}
$$

Suppressing the parameter $k$ we define

$$
\text { relocate } 1(m, z, y) \stackrel{\text { def }}{\equiv} y=k(z) \triangleright \bigcup_{x \in \operatorname{keys}(n) \backslash \hat{z}} \operatorname{fibre}(m, x, k(x))
$$

with the embedding

$$
\operatorname{relocate}(m, k) \equiv \operatorname{relocate} 1(m, \square, \square) .
$$

Immediately we obtain the recursion 


$$
\begin{aligned}
& \text { relocate1 }(m, z, y) \\
& \equiv y=k(z) \triangleright \text { if } \operatorname{next}_{n}(z) \notin \text { keys } \\
& \text { then } \emptyset \\
& \text { else } \operatorname{fibre}\left(m, \operatorname{next}_{n}(z), y+\operatorname{size}(z)\right) \cup \\
& \quad \operatorname{relocate} 1\left(m, \operatorname{next}_{n}(z), y+\operatorname{size}(z)\right) \mathrm{fi} .
\end{aligned}
$$

Because we are given keys + rather than $k e y s_{+}(n)$, we want to derive an algorithm based on

$$
\operatorname{next}(x) \stackrel{\text { def }}{=} \operatorname{succ}_{\text {keys }_{+}}(x)
$$

rather than on $\operatorname{ext}_{n}$. To this end we introduce

$$
\operatorname{pred}_{n}(x) \stackrel{\text { def }}{\equiv} \sup (\operatorname{keys}(n) \cap \hat{x} \backslash\{x\})
$$

and define

$$
\text { relocate2 }(m, z, y, x) \stackrel{\text { def }}{\equiv} x \in \text { keys }_{+} \wedge \operatorname{pred}_{n}(x)=z \triangleright \operatorname{relocate} 1(m, z, y)
$$

with the embedding

$$
\text { relocate }(m, k) \equiv \operatorname{relocate} 1(m, \square, \square) \equiv \operatorname{relocate} 2(m, \square, \square, \square+1) .
$$

Now assume $\operatorname{pred}_{n}(x) \equiv z$.

Case 1: $x \equiv$ bound.

Then $z \equiv \operatorname{bound}(n)$ and therefore relocate1 $(m, z, y) \equiv \emptyset$.

Case 2: $x \in$ keys $\backslash$ keys $(n)$.

Then $\operatorname{pred}_{n}(\operatorname{next}(x)) \equiv \operatorname{pred}_{n}(x) \equiv z$ and thus

$$
\text { relocate2 }(m, z, y, x) \equiv \text { relocate2 }(m, z, y, \operatorname{next}(x)) \text {. }
$$

Case 3: $x \in \operatorname{keys}(n)$.

Then $\operatorname{pred}_{n}(\operatorname{next}(x)) \equiv x \equiv \operatorname{next}_{n}(z)$. Therefore, by the recursion for relocate1,

$$
\begin{aligned}
& \text { relocated }(m, z, y, x) \\
\equiv & \operatorname{relocate} 1(m, z, y) \\
\equiv & \operatorname{fibre}(m, x, y+\operatorname{size}(z)) \cup \operatorname{relocate} 1(m, x, y+\operatorname{size}(z)) \\
\equiv & \operatorname{fibre}(m, x, y+\operatorname{size}(z)) \cup \operatorname{relocate} 2(m, x, y+\operatorname{size}(z), \operatorname{next}(x)) .
\end{aligned}
$$

Thus, defining

$$
R(m, z, y, x) \stackrel{\text { def }}{=} x \in k e y s_{+} \wedge y=k(x) \wedge \operatorname{pred}_{n}(x)=z
$$

we get the recursion

$$
\begin{aligned}
& \text { relocate2 }(m, z, y, x) \\
& \equiv \begin{array}{l}
R(m, z, y, x) \triangleright \\
\text { if } x=\text { bound } \\
\text { then } \emptyset \\
\text { else if } x \notin \operatorname{keys}(n) \\
\text { then } \operatorname{relocate} \mathcal{L}(m, z, y, \operatorname{next}(x)) \\
\text { else cell } y_{1} \equiv y+\operatorname{size}(z) ; \\
\quad \text { cell } x_{1} \equiv \operatorname{next}(x) ; \\
\quad \text { fibre }\left(m, x, y_{1}\right) \cup \operatorname{relocate} 2\left(m, x, y_{1}, x_{1}\right) \text { fi fi . }
\end{array}
\end{aligned}
$$


The body of the function copass is, like the body of relocate, a union over the index set keys $(n)$. Thus, forming copass requires a second traversal of keys $(n)$ and therefore of keys since we only have keys ( and not keys $(n)$ ) as a primitive. But if we compute next $_{n}$ (as a map) simultaneously with relocate, we can afterwards use it to traverse keys $(n)$, thus improving speed efficiency considerably. To this end we define

$$
\begin{aligned}
& \operatorname{mapnext}(n) \stackrel{\text { def }}{\equiv}\left(\bigcup_{y \in \text { keys }(n) \cup\{\square\}}\left[y \mapsto \operatorname{ext}_{n}(y)\right]\right) \cup[\operatorname{bound}(n) \mapsto \square] \\
& \equiv\left(\bigcup_{y \in \text { keys }(n)}\left[\operatorname{pred}_{n}(y) \mapsto y\right]\right) \cup \\
& {\left[\operatorname{pred}_{n}\left(\operatorname{bound}_{(}(n)\right) \mapsto \operatorname{bound}(n)\right] \cup[\operatorname{bound}(n) \mapsto \square] . }
\end{aligned}
$$

Then

$$
\operatorname{mapnext}(n)(y) \equiv \operatorname{next}_{n}(y) \text { for } y \in \operatorname{keys}(n) \cup\{\square\} \quad\left(\equiv \downarrow n \operatorname{ext}_{n}\right) .
$$

Applying similar techniques as in the case of relocate, we generalize mapnext to

$$
\begin{aligned}
\operatorname{mapnext1}(n, z, x) \stackrel{\text { def }}{\equiv} & x \in \text { keys }_{+} \wedge z=\operatorname{pred}_{n}(x) \triangleright \\
& \left(\bigcup_{y \in \operatorname{keys}(n) \backslash \hat{z}}\left[\operatorname{pred}_{n}(y) \mapsto y\right]\right) \cup \\
& {\left[\operatorname{pred}_{n}(\operatorname{bound}(n)) \mapsto \operatorname{bound}(n)\right] \cup[\operatorname{bound}(n) \mapsto \square] }
\end{aligned}
$$

with the embedding

$$
\operatorname{mapnext}(n) \equiv \operatorname{mapnext1}(n, \square, n)
$$

We obtain the recursion

$$
\begin{aligned}
& \text { mapnext1 }(n, z, x) \\
& \equiv x \in \text { keys }_{+} \wedge z=\operatorname{pred}_{n}(x) \triangleright \\
& \text { if } x=\text { bound } \\
& \text { then }[z \mapsto n \operatorname{ext}(z)] \cup[n \operatorname{ext}(z) \mapsto \square] \\
& \text { else if } x \notin \operatorname{keys}(n) \\
& \text { then mapnext } 1(n, z, n \operatorname{ext}(x)) \\
& \text { else mapnext } 1(n, x, n \operatorname{ext}(x)) \cup[z \mapsto x] \mathrm{fi} \mathrm{fi} \text {. }
\end{aligned}
$$

The recursions for relocate 2 and mapnext 1 fit together nicely. Furthermore, $\downarrow$ relocate $2(n, z, y, x) \subseteq$ $\downarrow \operatorname{arcs}(n)$ and $\downarrow$ mapnext $1(n, z, x) \subseteq$ keys $_{+}(n) \cup\{\square\}$, which implies that these domains are disjoint. Hence we may define

$$
\text { relocate3 }(m, z, y, x) \stackrel{\text { def }}{\equiv} \text { relocate2 }(n, z, y, x) \cup \operatorname{mapnext} 1(m, z, x)
$$

and obtain by function combination (see [Berghammer, Ehler 90]) the recursion

$$
\begin{aligned}
& \text { relocate3 }(m, z, y, x) \\
& \equiv R(m, z, y, x) \triangleright \\
& \text { if } x=\text { bound } \\
& \text { then }[z \mapsto n \operatorname{ext}(z)] \cup[n \operatorname{ext}(z) \mapsto \square] \\
& \text { else if } x \notin \operatorname{keys}(n) \\
& \text { then relocate3 }(m, z, y, n \operatorname{ext}(x)) \\
& \text { else cell } y_{1} \equiv y+\operatorname{size}(z) \text {; } \\
& \text { cell } x_{1} \equiv \operatorname{next}(x) \text {; } \\
& \text { fibre }\left(m, x, y_{1}\right) \cup[z \mapsto x] \cup \text { relocate } 3\left(m, x, y_{1}, x_{1}\right) \text { fi fi . }
\end{aligned}
$$


We have

$$
\text { relocate }(m, k) \cup \text { mapnext }(m) \equiv \operatorname{relocate3}(m, \square, \square, \square+1) \text {. }
$$

Now we transform relocate 3 in such a way that it works on $l$ rather than on $m \equiv$ retrieve $(l)$. Recall that

$$
\text { retrieve }(l) \equiv l|\downarrow m \equiv l|(\bigcup\{\text { ginner }(l, z) \mid z \in \text { keys } \wedge l(z) \not \equiv \square\})
$$

and $\operatorname{keys}(n) \equiv \operatorname{src}(m)$ since $n \equiv \operatorname{unchain}(m)$. Thus, for $x \in k e y s_{+}$,

$$
x \notin \operatorname{keys}(n) \Leftrightarrow l(z) \equiv
$$

and

$$
\operatorname{fibre}(m, x, y) \equiv \operatorname{fibre}(l, x, y)
$$

Hence we obtain for

$$
\text { relocate } 4(l, z, y, x) \stackrel{\text { def }}{\equiv} \text { relocate3 }(\operatorname{retrieve}(l), z, y, x)
$$

the recursion

$$
\begin{aligned}
& \text { relocate } 4(l, z, y, x) \\
& \equiv R(\text { retrieve }(l), z, y, x) \triangleright \\
& \text { if } x=\text { bound } \\
& \text { then }[z \mapsto n \operatorname{ext}(z)] \cup[n \operatorname{ext}(z) \mapsto \square] \\
& \text { else if } l(x)= \\
& \text { then relocate } 4(l, z, y, n \operatorname{ext}(x)) \\
& \text { else cell } y_{1} \equiv y+\operatorname{size}(z) \text {; } \\
& \text { cell } x_{1} \equiv \operatorname{next}(x) \text {; } \\
& \text { fibre }\left(l, x, y_{1}\right) \cup[z \mapsto x] \cup \text { relocate } 4\left(l, x, y_{1}, x_{1}\right) \text { fi fi . }
\end{aligned}
$$

Since for the initial call relocate $4(l, \square, \square, \square+1)$ the assertion is satisfied, we may omit it (cf. [Möller 89] for a formal treatment of this step). Furthermore we may add an accumulating parameter $q$ to obtain a tail-recursive form. Finally — to prepare overwriting — we replace in the second recursive call $l$ by $l \ominus l^{*}(x)$ where

$$
l^{*}(x) \stackrel{\text { def }}{=} \bigcup_{i \in \mathbb{N}} x \uparrow l^{i}(x)
$$

this does not affect the algorithm. The result is

$$
\begin{aligned}
& \text { relocate } 5(l, z, y, x, q) \\
& \equiv R \text { (retrieve }(l), z, y, x) \triangleright \\
& \text { if } x=\text { bound } \\
& \text { then } q \cup[z \mapsto n \operatorname{ext}(z)] \cup[n \operatorname{ext}(z) \mapsto \square] \\
& \text { else if } l(x)=\square \\
& \text { then relocate } 5(l, z, y, \operatorname{next}(x), q) \\
& \text { else cell } y_{1} \equiv y+\operatorname{size}(z) \text {; } \\
& \text { cell } x_{1} \equiv \operatorname{next}(x) \text {; } \\
& \text { state } l_{1} \equiv l \ominus l^{*}(x) \text {; } \\
& \text { state } q_{1} \equiv q \cup \text { fibre }\left(l, x, y_{1}\right) \cup[z \mapsto x] \text {; } \\
& \text { relocate } 5\left(l_{1}, x, y_{1}, x_{1}, q_{1}\right) \text { fi fi . }
\end{aligned}
$$

Defining

$$
\text { nrelocate }(l) \stackrel{\text { def }}{\equiv} \text { relocate }(\text { retrieve }(l), k) \cup \operatorname{mapnext}(n),
$$


where $n$ is the state to be compressed, we obtain

$$
\text { nrelocate }(l) \equiv \text { relocate } 5(l, \square, \square, \square+1, \emptyset)
$$

Obviously the property $\downarrow l \cap \downarrow q=\emptyset$ is an invariant for relocate 5 . Thus we may carry $l \cup q$ on a new parameter $l q$. This gives our final version

$$
\begin{aligned}
& \text { relocate } 6(l q, z, y, x) \\
& \equiv \text { if } x=\text { bound } \\
& \text { then } l q \cup[z \mapsto \operatorname{next}(z)] \cup[\operatorname{next}(z) \mapsto \square] \\
& \text { else if } l q(x)=\square \\
& \text { then relocate } 6(l q, z, y, \operatorname{next}(x)) \\
& \text { else cell } y_{1} \equiv y+\operatorname{size}(z) ; \\
& \quad \text { cell } x_{1} \equiv \operatorname{next}(x) ; \\
& \text { state } l q_{1} \equiv l q \triangleleft\left(\text { fibre }\left(l q, x, y_{1}\right) \cup[z \mapsto x]\right) ; \\
& \text { relocate } 6\left(l q_{1}, x, y_{1}, x_{1}\right)
\end{aligned}
$$$$
\text { fi fi . }
$$

relocate 6 satisfies

$$
l \nLeftarrow \text { nrelocate }(l) \equiv \text { relocate } 6(l, \square, \square, \square+1) \stackrel{\text { def }}{=} \text { onrelocate }(l)
$$

for $l$ such that retrieve $(l)$ is a chained map representation.

\subsection{Copying Pass}

We have seen that the relocation pass returns a union of the state $p \stackrel{\text { def }}{\equiv}$ relocate $(m, k)$ and the map $l \stackrel{\text { def }}{=} \operatorname{mapnext}(n)$ where

$$
p \equiv \operatorname{relocate}(m, k) \equiv \bigcup_{z \in \text { keys }(n)} \operatorname{fibre}(m, z, k(z))
$$

and

$$
l(y) \equiv \operatorname{mapnext}(n)(y) \equiv \operatorname{next}_{n}(y)
$$

for $y \in \operatorname{keys}(n) \cup\{\square\}$. We recall that $l(\square)$ is the minimum of keys $(n)$ and $l($ bound $(n)) \equiv \square$. Moreover, $\downarrow p \cap \downarrow l \equiv \emptyset$ making the union $p \cup l$ a well-defined map. We set $l p \stackrel{\text { def }}{=} p \cup l$. Now we have to construct $n_{c}$ from $l_{p}$ based on the values size $(z)$ for $z \in \operatorname{keys}(n)$; we call the corresponding function copypass, so that

$$
n_{c} \equiv \operatorname{copypass}(l p) .
$$

As stated before, $n_{c}$ is represented by

$$
n_{c} \equiv \bigcup_{z \in \text { keys }(n)}\left([k(z) \mapsto \square] \cup \bigcup_{i \in[1: s i z e(z)]}[k(z)+i \mapsto p(z+i)]\right)
$$

where now $l p$ can be substituted for $p$. We define, for $z \in \operatorname{keys}(n)$,

$$
\text { fibrecopass } \left.(z, \operatorname{lp}, k(z)) \stackrel{\text { def }}{=}[k(z) \mapsto \square] \cup \bigcup_{i \in[1: \operatorname{size}(z)]}[k(z)+i \mapsto \operatorname{lp}(z+i)]\right)
$$

and

$$
\text { fibrecopass }(\square, l p, \square) \stackrel{\text { def }}{\equiv} \emptyset \text {. }
$$


Next, for $y \in k e y s_{+}(n) \cup\{\square\}$ we define

$$
\operatorname{copass1}(y, l p, k) \stackrel{\text { def }}{=} \bigcup_{z \in \operatorname{keys}(n) \backslash y} \operatorname{fibrecopass}(z, \operatorname{lp}, k(z)) .
$$

Then

$$
n_{c} \equiv \operatorname{copass1}(\operatorname{bound}(n), l p, k) .
$$

We have the following recursion equations:

$$
\begin{aligned}
& \operatorname{copass}_{1}\left(\operatorname{next}_{n}(\square), l p, k\right) \equiv \emptyset, \\
& \operatorname{copass1}\left(\operatorname{next}_{n}(y), l p, k\right) \equiv \operatorname{copass1}(y, l p, k) \cup \operatorname{fibrecopass}(y, l p, k(y))
\end{aligned}
$$

for $y \not \equiv \square$. Embedding with an accumulator $n c$ leads to the "ascending" recursion

$$
\begin{aligned}
& \text { copass2 }(n c, y, l p, k) \\
& \equiv n c=\operatorname{copass1}(y, l p, k) \triangleright \\
& \text { if } y=\operatorname{bound}(n) \\
& \text { then } n c \\
& \text { else copass2( } \left.n c \cup \text { fibrecopass }(y, l p, k(y)), \operatorname{next}_{n}(y), l p, k\right) \mathrm{fi}
\end{aligned}
$$

with

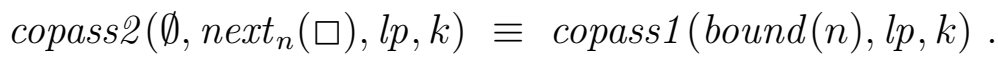

The last equation is a consequence of the recursion equations and of the finiteness of the function next $_{n}$. Hence we can compute $n_{c}$ as

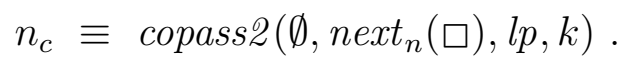

Obviously, the collapsing map $k$ is only used in computing fibrecopass $(y, l p, k(z))$; moreover, in this $k$ is only used to yield $k(y)$. As already shown in Section 3.2, $k$ satisfies the recursion equation

$$
k\left(\operatorname{next}_{n}(y)\right) \equiv k(y)+\operatorname{size}(y)
$$

for $y \in \operatorname{keys}(n)$ such that $\operatorname{next}_{n}(y) \not \equiv$ bound $(n)$. Hence we can eliminate $k$ and use its values on the single cells instead. Second, copass2 $(n c, y, l p, k)$ in fact only depends on the restriction of $l p$ to $\stackrel{v}{y}$. Therefore we can replace copass 2 by

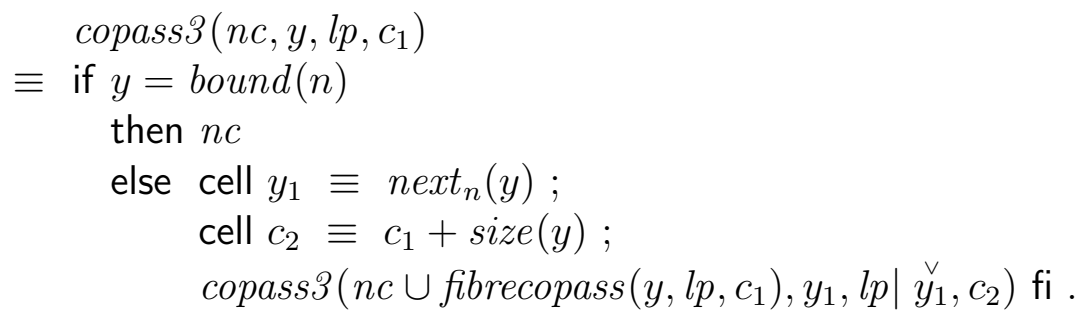

Furthermore, the condition $y=\operatorname{bound}(n)$ can be equivalently replaced by $l p(y) \equiv \square$; also, $l p(y)$ can be substituted for $\operatorname{next}_{n}(y)$, since $\operatorname{next}_{n}(y) \equiv l p(y)$ for all $y \in k e y s(n) \cup\{\square\}$.

For our last modification we observe that under the assertion $n c \cap l p \mid \breve{y_{1}}=\emptyset$ also

$$
\left(n c \cup \text { fibrecopass }\left(y, l p, c_{1}\right)\right) \cap l p \mid \stackrel{\vee}{y_{1}} \equiv \emptyset .
$$

Thus we can overwrite $l p$ using 


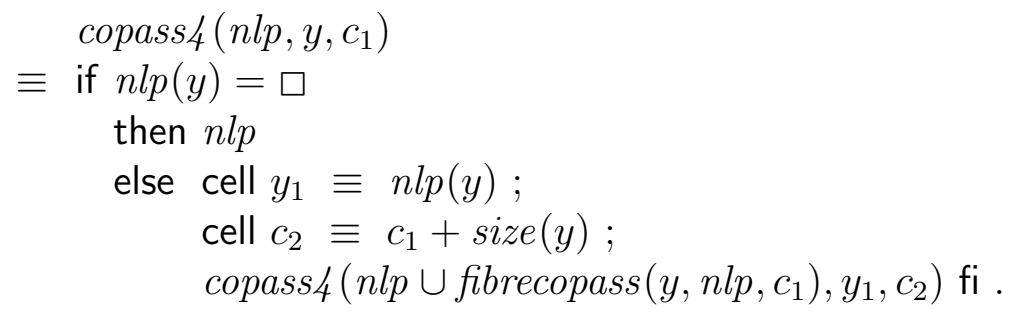

Now we are in the position to compute $n_{c}$ from $l p$ by a copy pass, viz.

$$
n_{c} \equiv \operatorname{copypass}(l p) \equiv n_{c}^{\prime} \mid\{|\downarrow n|\}^{\wedge}
$$

where

$$
n_{c}^{\prime} \stackrel{\text { def }}{\equiv} \operatorname{copass}_{4}\left(l p, \operatorname{next}_{n}(\square), \square+1\right) .
$$

Note that, in general, $n_{c} \not \equiv n_{c}^{\prime}$ since $n_{c}^{\prime}$ may contain parts of $l p$ that are not overwritten by copass 4 . It should also be clear that the computation of fibrecopass $\left(y, n l p, c_{1}\right)$ is elementary.

\subsection{Integrating the Relocation and Copying Passes}

Summarizing the results of the preceding sections we obtain the following: Assume that $m \equiv$ retrieve $(l)$ and $n \equiv$ unchain $(m)$. Then

$$
\begin{aligned}
& \operatorname{copypass}(\text { onrelocate }(l)) \\
\equiv & \operatorname{copypass}(l \& \text { nrelocate }(l)) \\
\supseteq & \operatorname{copass}(\operatorname{relocate}(m, k), k) \\
\equiv & \operatorname{copy}(n, k) \\
\equiv & n_{c} .
\end{aligned}
$$

Thus,

$$
\operatorname{ocopy}(l) \stackrel{\text { def }}{\equiv} \text { copypass }(\text { onrelocate }(l))
$$

is $n_{c}$, generally together with some garbage attached to the end of $n_{c}$ which, however, is easily deleted. Hence ocopy solves the copying part of our garbage collection problem. The complete program for it reads

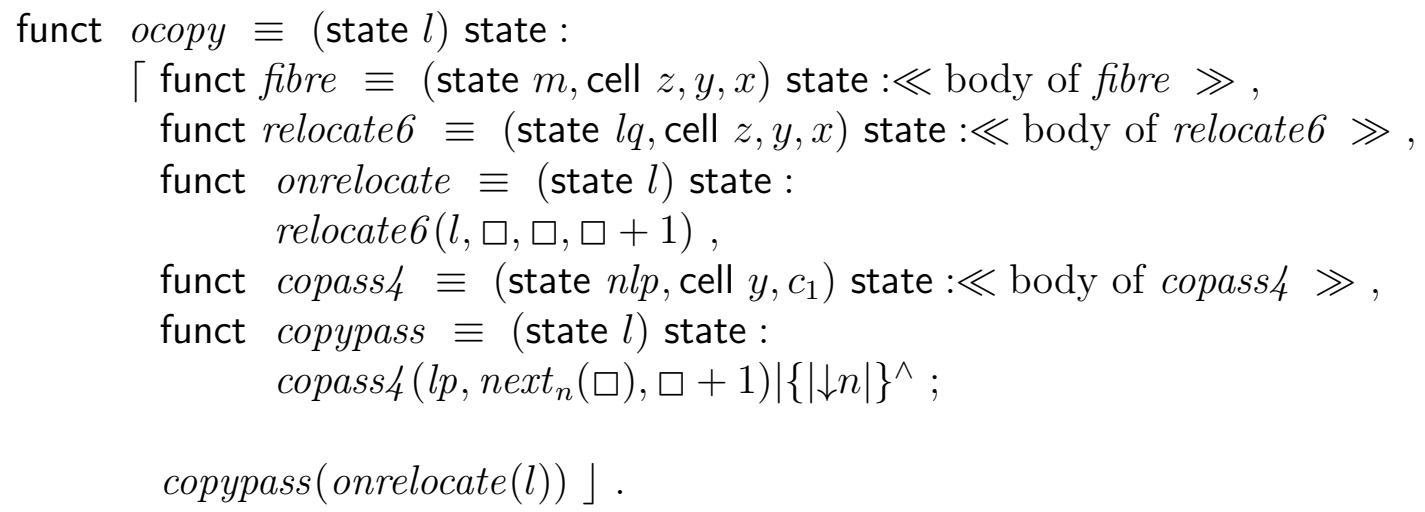

\section{Summary and Conclusion}

\subsection{The Complete Algorithm}

If we assemble the algorithms from Chapters 7 and 8, we obtain the following garbage collection program: 


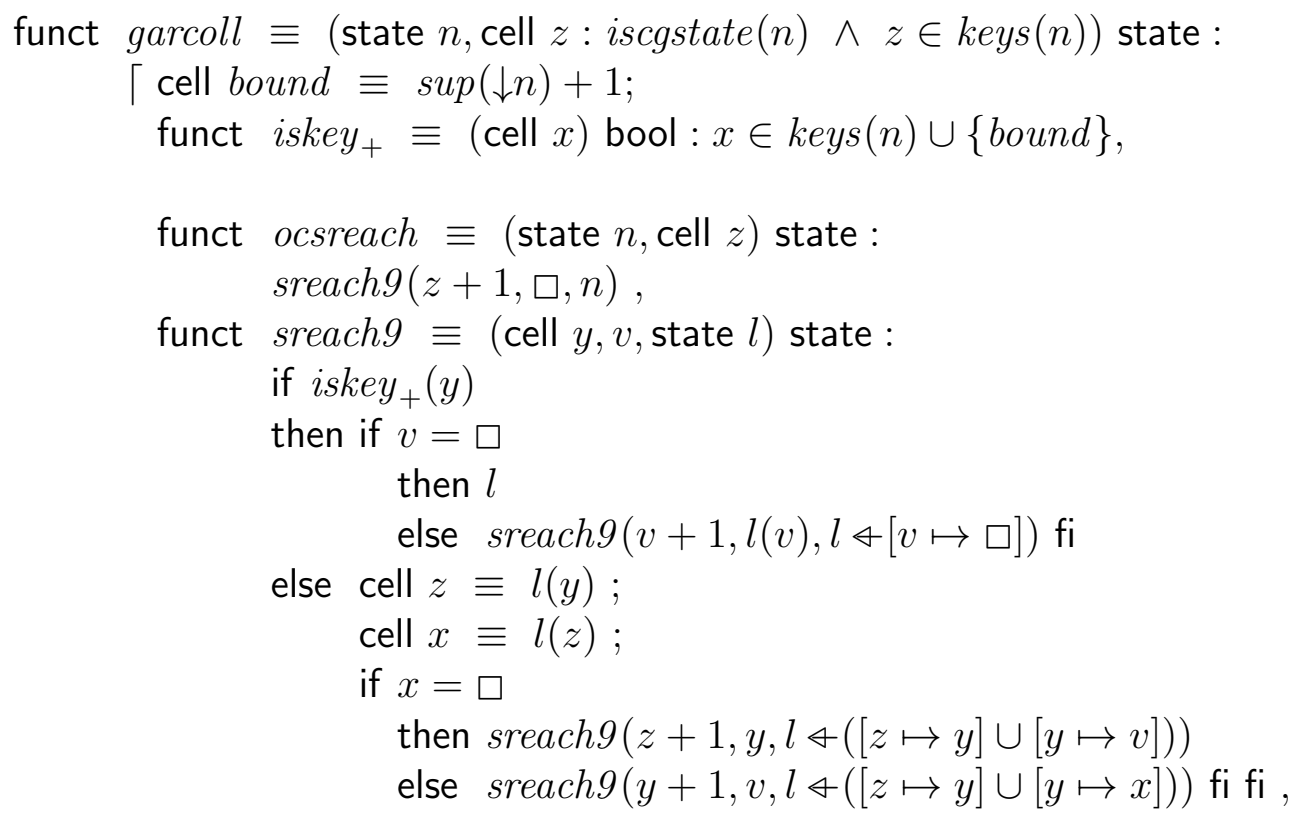

funct ocopy $\equiv($ state $l)$ state :

copypass (onrelocate $(l)$ ),

funct onrelocate $\equiv($ state $l)$ state :

relocate $6(l, \square, \square, \square+1)$,

funct relocate $6 \equiv$ (state $l q$, cell $z, y, x)$ state :

if $x=$ bound

then $l q \cup[z \mapsto \operatorname{next}(z)] \cup[\operatorname{next}(z) \mapsto \square]$

else if $l q(x)=$

$$
\begin{aligned}
& \text { then relocate } 6(l q, z, y, n \operatorname{ext}(x)) \\
& \text { else cell } y_{1} \equiv y+\operatorname{size}(z) ; \\
& \quad \text { cell } x_{1} \equiv \operatorname{next}(x) ; \\
& \text { state } l q_{1} \equiv l q \triangleleft\left(\text { fibre }\left(l q, x, y_{1}\right) \cup[z \mapsto x]\right) ; \\
& \text { relocate } 6\left(l q_{1}, x, y_{1}, x_{1}\right)
\end{aligned}
$$

funct fibre $\equiv$ (state $l$, cell $x, y:$ isanchored $(l) \wedge x \in \downarrow l)$ state :

$\lceil$ cell $z \equiv l(x)$;

if $z=\square$ then $\emptyset$ else $[z \mapsto y] \cup \operatorname{fibre}(l, z, y)$ fi $\rfloor$,

funct copypass $\equiv$ (state $l$ ) state :

$\operatorname{copass}_{4}\left(\operatorname{lp}, \operatorname{next}_{n}(\square), \square+1\right) \mid\{|\downarrow n|\}^{\wedge} ;$

funct copass $4 \equiv$ (state $n l p$, cell $\left.y, c_{1}\right)$ state :

if $n l p(y)=$

then $n l p$

else cell $y_{1} \equiv n \operatorname{lp}(y)$;

cell $c_{2} \equiv c_{1}+\operatorname{size}(y)$;

copass $4\left(n l p \cup\right.$ fibrecopass $\left.\left(y, n l p, c_{1}\right), y_{1}, c_{2}\right)$ fi ;

ocopy $(\operatorname{ocsreach}(n, z))$

In this version the compressed state

$$
n_{c} \stackrel{\text { def }}{\equiv} \operatorname{garcoll}(n, z)
$$

may still contain garbage in the cells greater than bound $\left(n_{c}\right)$. But this does not matter because we can easily calculate bound $\left(n_{c}\right)$ and thus we know which cells are free for subsequent re-use. The computation of bound $\left(n_{c}\right)$ can even be combined with the routine relocate 6 , since in the 
termination case $x=$ bound we have bound $\left(n_{c}\right) \equiv y+\operatorname{size}(z)$. As a final step, one can now pass immediately to a procedural version of this algorithm and thus introduce selective updating. Note that no further development at that level is necessary.

\subsection{Discussion}

The algebra of maps has proved to be an invaluable tool in our derivation of the garbage collection algorithm. It has allowed us to carry out the development in full detail without leading to unmanageably complex expressions. It also has allowed us to stay entirely at the applicative language level where calculation is easy due to the strong algebraic properties of applicative languages. We are convinced that this way of approaching machine-oriented programs is an important step towards achieving systems software with guaranteed correctness.

It is to be hoped that the theory about states and chained representations developed in this paper can be re-used for further transformational studies of pointer algorithms. Apart from this, the development shows the importance and feasibility of early modularization of a specification; the transformational approach is strong enough to allow efficiency-increasing integration of program parts that have been developed independently.

\section{Acknowledgement}

We are grateful to R. Tobiasch for drawing our attention to the garbage collection problem. Valuable comments and suggestions concerning this paper have been provided by F.L. Bauer, R. Berghammer, R. Dewar, W. Dosch, M. Lichtmannegger, H. Partsch, P. Pepper, M. Sintzoff, and, in particular, by H. Ehler. 


\section{References}

[Bauer, Wössner 82]

F.L. Bauer, H. Wössner: Algorithmic language and program development. New York: Springer 1982

[Bauer et al. 85]

F.L. Bauer et al.: The Munich project CIP. Volume I: The wide spectrum language CIP-L. Lecture Notes in Computer Science 183. New York: Springer 1985

[Berghammer, Ehler 90]

R. Berghammer, H. Ehler: On the use of elements of functional programming in program development by transformations. This volume

[Berghammer et al. 87]

R. Berghammer, H. Ehler, H. Zierer: Development of graph algorithms by program transformation. In: H. Göttler, H.-J. Schneider (eds.): Graph-theoretic concepts in computer science. Lecture Notes in Computer Science 314. Berlin: Springer 1988, 206-218

[Broy, Pepper 82]

M. Broy, P. Pepper: Combining algebraic and algorithmic reasoning: An approach to the SchorrWaite-Algorithm. ACM TOPLAS 4, 362-381 (1982)

[Dewar, McCann 77]

R. Dewar, A. McCann: MACRO SPITBOL - a SNOBOL4 compiler. Software — Practice and Experience 7, 95-113 (1977)

[Dewar et al. 82]

R. Dewar, M. Sharir, E. Weixelbaum: Transformational derivation of a garbage collection algorithm. ACM TOPLAS 4, 650-667 (1982)

[van Diepen, de Roever 86]

N. van Diepen, W. de Roever: Program derivation through transformations: The evolution of list-copying algorithms. Science of Computer Programming 6, 213-272 (1986)

[Möller 87]

B. Möller: Entwicklung eines Speicherbereinigungsalgorithmus. Institut für Informatik der TU München, Manuscript, October 1987

[Möller 89]

B. Möller: Applicative assertions. In : J.L.A. van de Snepscheut (ed.): Mathematics of Program Construction, Groningen, 26-30 June 1989. Lecture Notes in Computer Science 375. Berlin: Springer 1989, 348-362

[Möller 90]

B. Möller: Formal derivation of pointer algorithms. In: M. Broy (ed.): Informatik im Kreuzungspunkt von Numerischer Mathematik, Rechnerentwurf, Programmierung, Algebra und Logik. Festkolloquium, München, 12.-14. Juni 1989. Lecture Notes in Computer Science (to appear) 


\section{Appendix: Some Properties of Map Operations}

All properties in this appendix are stated without proof. We group them according to the map operations they involve.

\subsection{Domain and Range}
(1) $\quad t \downarrow m \subseteq \downarrow m$
(2) $s \uparrow m \subseteq \uparrow m$
(3) $s \subseteq t \Rightarrow s \downarrow m \subseteq t \downarrow m$
(4) $s \subseteq t \Rightarrow s \uparrow m \subseteq t \uparrow m$
(5) $\overline{s \downarrow m} \equiv \downarrow m \backslash s \downarrow m \cup \overline{\downarrow m}$
(6) $\quad(\uparrow m) \backslash t \equiv \overline{t \downarrow m \uparrow m}$
(7) $s \subseteq \downarrow m \Rightarrow(s \uparrow m) \downarrow m \supseteq s$
(8) $\quad t \subseteq \uparrow m \Rightarrow(t \downarrow m) \uparrow m \equiv t$
(9) $s \subseteq t \downarrow m \Rightarrow s \uparrow m \subseteq t$
(10) $t \supseteq s \uparrow m \Rightarrow t \downarrow m \supseteq s$
(11) $(\uparrow m) \downarrow m \equiv \downarrow m$
(12) $(\downarrow m) \uparrow m \equiv \uparrow m$
(13) $s \downarrow(m \cup n) \equiv s \downarrow m \cup s \downarrow n$
(14) $\downarrow(m \cup n) \equiv \downarrow m \cup \downarrow n$
(15) $t \uparrow(m \cup n) \equiv t \uparrow m \cup t \uparrow n$
(16) $\uparrow(m \cup n) \equiv \uparrow m \cup \uparrow n$
(17) $m \subseteq n \Rightarrow s \downarrow m \subseteq s \downarrow n$
(18) $m \subseteq n \Rightarrow s \uparrow m \subseteq s \uparrow n$

\subsection{Restriction}
(1) $m \mid s \subseteq m$
(3) $s \subseteq \bar{\downarrow} m \Rightarrow m \mid s \equiv \emptyset$
(5) $m \mid \emptyset \equiv \emptyset$
(7) $\downarrow m \subseteq s \Rightarrow m \mid s \equiv m$
(9) $\quad m \mid \downarrow m \equiv m$
(11) $(m \mid s)|t \equiv m|(s \cap t)$
(13) $m|(s \cup t) \equiv m| s \cup m \mid t$
(15) $m|(s \cap t) \equiv m| s \cap m \mid t$
(17) $m|(s \backslash t) \equiv m| s \cap m \ominus t$
(19) $t \downarrow(m \mid s) \equiv(t \downarrow m) \cap s$
(21) $\downarrow(m \mid s) \equiv \downarrow m \cap s$
(23) $t \uparrow(m \mid s) \equiv(t \cap s) \uparrow m$
(25) $\uparrow(m \mid s) \equiv s \uparrow m$
(27) $(m \cup n)|s \equiv m| s \cup n \mid s$

(2) $m \ominus s \subseteq m$

(4) $s \subseteq \overline{\downarrow m} \Rightarrow m \ominus s \equiv m$

(6) $m \ominus \emptyset \equiv m$

(8) $\downarrow m \subseteq s \Rightarrow m \ominus s \equiv \emptyset$

(10) $m \ominus \downarrow m \equiv \emptyset$

(12) $(m \ominus s) \ominus t \equiv m \ominus(s \cup t)$

(14) $m \ominus(s \cup t) \equiv m \ominus s \cap m \ominus t$

(16) $m \ominus(s \cap t) \equiv m \ominus s \cup m \ominus t$

(18) $m \ominus(s \backslash t) \equiv m \ominus s \cup m \mid t$

(20) $t \downarrow(m \ominus s) \equiv(t \downarrow m) \backslash s$

(22) $\downarrow(m \ominus s) \equiv \downarrow m \backslash s$

(24) $t \uparrow(m \ominus s) \equiv(t \backslash s) \uparrow m$

(26) $\uparrow(m \ominus s) \equiv \bar{s} \uparrow m$

(28) $(m \cup n) \ominus s \equiv m \ominus s \cup m \ominus t$

\subsection{Overwriting}

(1) $\emptyset \triangleleft m \equiv m \triangleleft \emptyset \equiv m$

(2) $(l \nLeftarrow m) \triangleleft n \equiv l \nLeftarrow(m \varangle n)$

(3) $m \varangle n \equiv n \nleftarrow m$ iff $m$ and $n$ are compatible.

In this case, $m \varangle n \equiv m \cup n$.

(4) $s \downarrow(m \varangle n) \equiv(s \downarrow m \backslash \downarrow n) \cup s \downarrow n$

(5) $\downarrow(m \triangleleft n) \equiv \downarrow m \cup \downarrow n$

(6) $m \varangle n \equiv n \Leftrightarrow \downarrow m \subseteq \downarrow n$

(7) $m \varangle n \equiv m \Leftrightarrow n \subseteq m$

(8) $(m \triangleleft n) \ominus s \equiv(m \ominus(\downarrow n \cup s)) \cup n \ominus s \equiv(m \ominus(\downarrow n) \ominus s) \cup n \ominus s$

(9) $\uparrow(m \varangle n) \equiv \overline{\downarrow n} \uparrow m \cup \uparrow n$ 